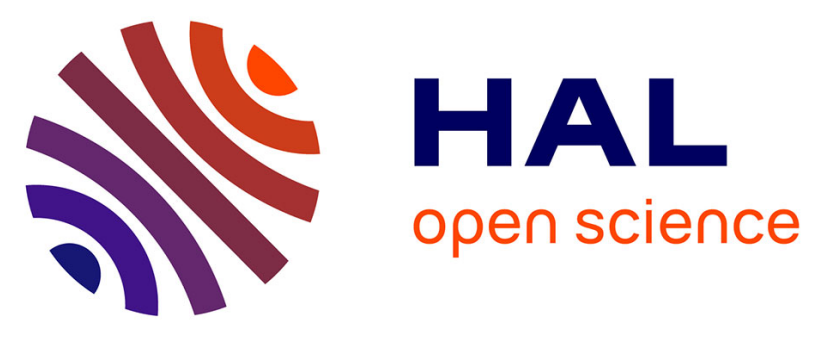

\title{
Plant Small RNA Species Direct Gene Silencing in Pathogenic Bacteria as well as Disease Protection
}

\author{
Meenu Singla-Rastogi, Magali Charvin, Odon Thiebeauld, Alvaro L \\ Perez-Quintero, Antinéa Ravet, Antonio Emidio-Fortunato, Venugopal \\ Mendu, Lionel Navarro
}

\section{To cite this version:}

Meenu Singla-Rastogi, Magali Charvin, Odon Thiebeauld, Alvaro L Perez-Quintero, Antinéa Ravet, et al.. Plant Small RNA Species Direct Gene Silencing in Pathogenic Bacteria as well as Disease Protection. 2019. hal-02422538

\section{HAL Id: hal-02422538 \\ https://hal.science/hal-02422538}

Preprint submitted on 22 Dec 2019

HAL is a multi-disciplinary open access archive for the deposit and dissemination of scientific research documents, whether they are published or not. The documents may come from teaching and research institutions in France or abroad, or from public or private research centers.
L'archive ouverte pluridisciplinaire HAL, est destinée au dépôt et à la diffusion de documents scientifiques de niveau recherche, publiés ou non, émanant des établissements d'enseignement et de recherche français ou étrangers, des laboratoires publics ou privés. 


\section{Plant Small RNA Species Direct Gene Silencing in Pathogenic Bacteria as well as Disease Protection}

Meenu Singla-Rastogi ${ }^{1}$, Magali Charvin ${ }^{1 \S}$, Odon Thiébeauld ${ }^{2 \S}$, Alvaro L Perez-Quintero ${ }^{1}$ Antinéa Ravet ${ }^{1}$, Antonio Emidio-Fortunato ${ }^{2}$, Venugopal Mendu ${ }^{1} \&$ Lionel Navarro $^{1 *}$

${ }^{1}$ Institut de Biologie de l'Ecole Normale Supérieure (IBENS), 75005 Paris, France; Centre National de la Recherche Scientifique UMR8197, 75005 Paris France, Institut National de la Santé et de la Recherche Médicale U1024, 75005 Paris, France.

${ }^{2}$ ImmunRise, 46 Rue d'Ulm, 75005 Paris, France.

$\S$ These authors contributed equally to the work

*To whom correspondence should be addressed: lionel.navarro@ens.fr 


\begin{abstract}
Plant small RNAs (sRNAs) and/or double-stranded RNAs (dsRNAs) trigger RNA interference (RNAi) in interacting eukaryotic pathogens or parasites. However, it is unknown whether this phenomenon could operate in bacterial phytopathogens, which lack a eukaryoticlike RNAi machinery. Here, we first show that Arabidopsis-encoded inverted repeat transgenes trigger silencing of Pseudomonas syringae heterologous reporter and endogenous virulence-associated genes during infection. Antibacterial Gene Silencing (AGS) of the latter was associated with a reduced pathogenesis, which was also observed upon application of corresponding plant-derived RNAs onto wild-type plants prior to infection. We additionally demonstrate that sRNAs directed against virulence factor transcripts were causal for silencing and pathogenesis reduction, while cognate long dsRNAs were inactive. Overall, this study provides the first evidence that plant sRNAs can directly reprogram gene expression in a phytopathogenic bacterium and may have wider implications in the understanding of how plants regulate transcriptome, community composition and genome evolution of associated bacteria.
\end{abstract}

\title{
Introduction
}

RNAi is a conserved gene regulatory mechanism that has been characterized as an antiviral defense response by repressing translation, accumulation and/or replication of viral RNAs ${ }^{1}$. In plants, RNAi has also been shown to control resistance against bacterial, fungal and oomycete pathogens partly by fine-tuning the expression of immune-responsive genes ${ }^{2,3}$. The core mechanism of RNAi involves the processing of dsRNAs or single-stranded RNAs (ssRNAs) carrying stem loop structures (e.g. primary microRNA -miRNA- transcripts) by DCL proteins leading to the production of 20-25 nt long short interfering RNAs (siRNAs) or miRNAs. siRNAs or miRNAs are then loaded into Argonaute (AGO) proteins to direct posttranscriptional silencing of sequence complementary mRNA targets through endonucleolytic 
cleavage and/or translational inhibition ${ }^{4}$.

An important feature of plant sRNAs, and particularly of siRNAs, is their ability to trigger non-cell autonomous silencing in adjacent cells as well as in distal tissues ${ }^{5,6}$. This phenomenon is essential to prime antiviral response ahead of the infection front but also to translocate silencing signals between plant cells and their non-viral eukaryotic interacting (micro)organisms ${ }^{7,8}$. For example, plant sRNAs were previously found to be exported in the fungal pathogens Verticillium dahliae ${ }^{9}$ and Botrytis cinerea ${ }^{10}$ as well as in the oomycete pathogen Phytophthora capsici ${ }^{11}$, leading to the silencing of pathogenicity factors. On the other hand, fungal siRNAs from $B$. cinerea were shown to be translocated into plant cells to silence defense genes ${ }^{12}$, highlighting bidirectional RNAi in a natural plant-fungal interaction. In addition, a very recent report provides evidence that rhizobial tRNA-derived sRNAs can silence legume symbiotic genes ${ }^{13}$. However, whether plant sRNAs can in turn directly reprogram gene expression in plant-associated bacteria remains unknown.

Artificial trans-kingdom RNAi has long been employed to direct Host-Induced Gene Silencing (HIGS), a technology used to characterize the function of fungal and oomycete genes or to engineer disease resistance in plants. HIGS notably relies on in planta expression of dsRNAs bearing homologies to essential and/or virulence genes and can operate in insects, nematodes, parasitic plants, oomycete and fungi. For example, HIGS confers full protection against Fusarium graminearum ${ }^{14}$ and B. $_{\text {cinerea }}{ }^{10}$, a phenotype which can be recapitulated by spraying antifungal dsRNAs and/or siRNAs onto Arabidopsis wild type (WT) plants prior to infection $^{15,10}$. The latter phenomenon is referred to as Spray-Induced Gene Silencing (SIGS) and is reminiscent of 'environmental RNAi', a process involving the uptake of RNAs from the environment to trigger RNAi ${ }^{16,17}$. However, so far, HIGS and SIGS have only been shown to be functional in eukaryotic (micro)organisms possessing canonical RNA silencing factors. Indeed, there is currently no evidence indicating that host-encoded dsRNAs and/or sRNAs 
bioRxiv preprint first posted online Dec. 3, 2019; doi: http://dx.doi.org/10.1101/863902. The copyright holder for this preprint

could direct gene silencing in interacting phytopathogenic bacteria, which lack conventional eukaryotic-like RNAi factors. It is also unknown whether external application of any of these RNA entities could trigger environmental RNAi in pathogenic prokaryotic cells and if such hypothetical RNA-based regulatory process could be used to protect plants towards bacterial diseases. Here, we wanted to test these intriguing ideas using Pseudomonas syringae pv. tomato strain DC3000 (Pto DC3000) as an experimental model system.

\section{Results}

Pto DC3000 is a Gram-negative bacterium that is the causal agent of bacterial speck disease in tomato and can also infect Arabidopsis thaliana ${ }^{18}$. This bacterium enters into leaf tissues through stomata or wounds and further multiplies in the apoplast ${ }^{19}$. To assess whether plant sRNAs and/or dsRNAs could reprogram Pto DC3000 gene expression, we first targeted a chromosomally integrated Photorhabdus luminescens luxCDABE reporter driven by the constitutive kanamycin promoter ${ }^{20}$. This lux-tagged Pto DC3000 (Pto WT LUC) strain spontaneously emits luminescence because it co-expresses the luciferase catalytic components $\operatorname{lux} A$ and $\operatorname{lu} x B$ genes along with the genes required for substrate production, namely $\operatorname{lux} C$, $\operatorname{luxD}$ and $\operatorname{lux} E^{21}$ (Fig.1a). Two independent Arabidopsis transgenic lines that constitutively express a chimeric inverted repeat which carries sequence homology with the coding regions of $\operatorname{lux} A$ and $\operatorname{lux} B$ were selected on the basis of their ability to produce anti-luxA and anti-luxB siRNAs (Fig.1b). They were subsequently syringe-infiltrated with the lux-tagged Pto DC3000 strain and the levels of luminescence activity were monitored at 24 hours post-inoculation (hpi). A significant reduction in luminescence activity was found in IR- $L U X A / L U X B-$ compared to control Col-0-infected plants (Fig.1c). By contrast, the growth of the bacterial reporter strain remained unchanged in the apoplast of IR-LUXA/LUXB lines compared to Col0 plants (Fig.1d), indicating that the above effects were not due to a decreased bacterial titer 
in these transgenic lines. Furthermore, a significant reduction in the accumulation of both luxA and $\operatorname{luxB}$ mRNAs was found in IR- $L U X A / L U X B$ - compared to control Col-0-infected plants, while the levels of the non-targeted proC bacterial transcripts remained unchanged (Fig.1e). These data indicate that an Arabidopsis-encoded inverted repeat can trigger silencing of Pto DC3000 heterologous reporter genes during infection.

Pto DC3000 possesses a large repertoire of pathogenicity factors, among them the type III secretion system (TTSS) and the phytotoxin coronatine (COR), which mimics the most active isoleucine conjugate of the phytohormone Jasmonic Acid (JA), both being critical for pathogenesis $^{22-25}$. To investigate whether Antibacterial Gene Silencing (AGS) could be additionally effective against endogenous Pto DC3000 genes that are relevant for pathogenesis, we next generated Arabidopsis transgenic plants that constitutively express a chimeric inverted repeat carrying sequence homology with the coding regions of the coronafacic acid polyketide synthase I (cfa6) gene and of the hrpL alternative sigma factor of Pto DC3000 (Fig.2a). The former bacterial factor is essential for the biosynthesis of coronafacic acid (CFA), which is a major structural component of $\mathrm{COR}^{26}$, while the latter controls directly the expression of type III-secretion system associated genes and indirectly the expression of COR biosynthesis genes ${ }^{27,}{ }^{28}$. As a negative control, we have generated Arabidopsis transgenic lines overexpressing an inverted repeat, which does not exhibit sequence homology with the Pto DC3000 genome, but instead targets three cytochrome P450 lanosterol C-14 $\alpha$-demethylase (CYP51) genes of the fungal pathogen $F$. graminearum ${ }^{14}$. These stable transgenic lines are referred to as IR-CFA6/HRPL and control vector $(\mathrm{CV})$ plants, respectively, and do not exhibit any developmental defect compared to Col-0 plants, despite high accumulation of artificial siRNAs (Fig.2b, 2c; Supplementary Fig.2). Additional characterization of the reference IR-CFA6/HRPL\#4 transgenic line by sRNA sequencing, revealed high and comparable levels of anti-cfa6 and anti-hrpL siRNAs, with a bias towards 
bioRxiv preprint first posted online Dec. 3, 2019; doi: http://dx.doi.org/10.1101/863902. The copyright holder for this preprint

$21 \mathrm{nt}$ siRNAs (Fig.2d). Furthermore, siRNAs were produced along the CFA6 and HRPL regions of the chimeric inverted repeat (Fig.2e), which is consistent with a processive cleavage of the $C F A 6 / H R P L$ dsRNA precursors by DCL proteins. An sRNA target prediction analysis against the Arabidopsis thaliana and Pto DC3000 annotated genes also indicated that an off-target effect seems unlikely (Supplementary Fig.1, Table S1, S2).

We further dip-inoculated these transgenic plants with Pto DC3000 and monitored cfa6 and hrpL mRNA levels by RT-qPCR analyses at 3 days-post infection (dpi). While the $c f a 6$ mRNA levels were moderately altered in two out of three independent IR-CFA6/HRPL lines compared to Col-0 plants, the levels of $h r p L$ transcripts were reproducibly and significantly reduced in all the three IR-CFA6/HRPL lines compared to Col-0 plants (Fig.2f). By contrast, the down-regulation of $c f a 6$ or hrpL mRNAs was not observed in CV-versus Col-0-infected plants (Fig.2f), supporting a specific effect of these antibacterial RNAs. In addition, the mRNA level of the non-targeted proC gene was unchanged in IR-CFA6/HRPL-infected lines compared to Col-0- or CV-infected plants (Fig.2f). We conclude that the Arabidopsis-encoded IR-CFA6/HRPL transgene can at least trigger silencing of the Pto DC3000 hrpL gene during infection.

It has been previously reported that Pto DC3000 triggers the reopening of stomata as a counter-defense towards plant-induced stomatal closure, which is rapidly orchestrated upon bacterial detection $^{29}$. This virulence response is critical for Pto DC3000 pathogenesis as it allows bacterial cells to reach the apoplast and to colonize inner leaf tissues. Previous studies have also shown that COR plays a major role in Pto DC3000-induced stomatal reopening ${ }^{30}$. Accordingly, the COR-deficient Pto DC3118 strain, which is specifically mutated in the $c f a 6$ gene and referred to here as Pto DC3000 $\Delta c f a 6$ (Pto $\Delta c f a 6)$, is not able to reopen stomata at 3 hpi (Fig.3a, Supplementary Fig.3a). Similarly, we found that the Pto DC3000 $\Delta h r p L$ (Pto $\Delta h r p L)$ strain, which is also deficient in COR biosynthesis ${ }^{24}$, was not capable of 
reopening stomata (Fig.3a, Supplementary Fig.3a). On the contrary, and as shown previously $^{30}$, a normal stomatal reopening phenotype was observed during infection with the type III secretion-defective Pto DC3000hrcC mutant (Pto $\Delta h r c C$ ) (Fig.3a; Supplementary Fig.3a), indicating that at this infection timepoint, type III effectors appear to be dispensable for this response.

We next monitored Pto DC3000-induced stomatal reopening response in IR-CFA6/HRPL transgenic lines. Significantly, these plants were fully insensitive to the stomatal reopening response typically triggered by Pto DC3000 at 3 hpi (Fig.3b; Supplementary Fig.3b), thereby mimicking the phenotypes observed during infection of Col-0 plants with the Pto $\Delta c f a 6$ or the Pto $\Delta h r p L$ strains (Fig.3a, 3b; Supplementary Fig.3a, 3b). By contrast, Pto DC3000-triggered stomatal reopening events were unaltered in CV-compared to Col-0-infected plants (Fig.3c; Supplementary Fig.3c), supporting a specific effect of antibacterial RNAs in this process. In addition, the altered stomatal reopening phenotype detected in IR-CFA6/HRPL plants infected with the WT Pto DC3000 strain, and in Col-0 plants challenged with the Pto $\Delta c f a 6$ or the Pto $\Delta h r p L$ strains, was fully rescued upon exogenous application of COR (Fig.3a, 3b; Supplementary Fig.3a, 3b). These data provide pharmacological evidence that the reduced Pto DC3000 pathogenesis manifested at infected IR-CFA6/HRPL stomata is likely caused by an altered ability of the associated and/or surrounding bacterial cells to produce COR.

We next assessed the impact that AGS could have on the ability of Pto DC3000 to colonize the apoplast of surface-inoculated leaves, a phenotype which is dependent on both type III effectors and $\mathrm{COR}^{22}$. To this end, we dip-inoculated Col-0, CV and IR-CFA6/HRPL plants with Pto DC3000 and subsequently monitored bacterial titer at 2 dpi. We found that Pto DC3000 was less effective in colonizing the apoplast of IR-CFA6/HRPL lines compared to Col-0 and CV-infected plants, a phenotype that was comparable to the growth defect of the Pto $\Delta c f a 6$ strain in Col-0 plants (Fig.4a). Nevertheless, this phenotype was less pronounced 
than the one observed with the Pto $\Delta h r p L$ strain grown on Col-0 plants (Supplementary Fig.4a), which might be due to the partial silencing of the targeted virulence factors and/or to the known RNA silencing suppression activity triggered by Pto DC3000 (Fig.2f) ${ }^{31,32}$. In addition, we noticed that IR-CFA6/HRPL plants dip-inoculated with Pto DC3000 showed reduced water-soaking symptoms at 1 dpi (Fig.4b), thereby phenocopying Col-0 plants dip-

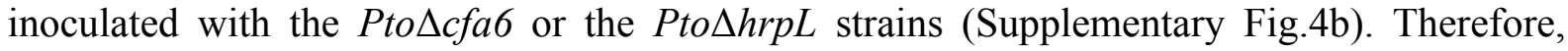
AGS can alter the ability of Pto DC3000 to trigger water-soaking and to multiply in the apoplast of Arabidopsis leaves, which are both critical steps of bacterial pathogenesis ${ }^{33}$.

Next, we assessed whether AGS could interfere with the capacity of Pto DC3000 to colonize xylem vessels, which is the main route used by this bacterium to propagate in the leaf vasculature $^{34,35}$. This Pto DC3000 virulence phenotype has been recently characterized and found here to be dependent on both type III effectors and COR (Supplementary Fig.4c, 4d). For this purpose, we wound-inoculated the leaf midvein of IR-CFA6/HRPL plants with a GFP-tagged Pto DC3000 (Pto DC3000-GFP) and further scored the number of bacterial propagation from inoculation sites. We found a strong decrease in Pto DC3000-GFP vascular propagation in the IR-CFA6/HRPL lines compared to Col-0- and CV-infected plants (Fig.4c, 4d), thereby mimicking the impaired spreading phenotype of the Pto $\Delta c f a 6-\mathrm{GFP}$ and Pto $\Delta h r p L$-GFP strains on Col-0 plants (Supplementary Fig.4c). Altogether, these data demonstrate that AGS can limit pathogenesis of Pto DC3000 in different tissues of Arabidopsis transgenic plants.

Environmental RNAi is a phenomenon by which (micro)organisms uptake external RNAs from the environment, resulting in the silencing of genes containing sequence homologies to the RNA triggers ${ }^{17}$. This process has been initially characterized in Caenorhabditis elegans ${ }^{36-}$ 39 and was further found to operate in other nematodes but also in insects, plants and fungi ${ }^{15,40}$. However, this approach has never been used against a bacterial phytopathogen that 
bioRxiv preprint first posted online Dec. 3, 2019; doi: http://dx. doi.org/10.1101/863902. The copyright holder for this preprint

lacks a canonical eukaryotic-like RNAi machinery such as Pto DC3000. To test this possibility, we first assessed whether RNA extracts from IR-CFA6/HRPL plants could trigger silencing of $c f a 6$ and $h r p L$ genes in in vitro conditions. For this purpose, we incubated total RNAs from CV and IR-CFA6/HRPL plants with Pto DC3000 cells, and further analyzed by RT-qPCR the levels of $c f a 6$ and $h r p L$ mRNAs. Results from these analyses revealed a reduction in the accumulation of both virulence factor mRNAs upon treatment with RNA extracts from IR-CFA6/HRPL plants (Fig.5a). By contrast, the level of the non-targeted proC and $г р о B$ mRNAs remained unaltered in the same conditions (Fig.5a). These data imply that plant antibacterial RNAs are likely taken-up by Pto DC3000 cells and subsequently trigger gene silencing in these prokaryotic cells. It also suggests that exogenous application of these antibacterial RNAs could be used as a strategy to dampen pathogenesis. To test this idea, we pre-treated Arabidopsis Col-0 leaf sections with total RNAs from IR-CFA6/HRPL plants for one hour, subsequently challenged them with Pto DC3000, and further monitored stomatal reopening events at 3 hpi. Strikingly, we found that RNA extracts from IR-CFA6/HRPL plants fully suppressed the ability of Pto DC3000 to reopen stomata (Fig.5b; Supplementary Fig.6a), thereby mimicking the phenotype observed in infected IR-CFA6/HRPL leaves (Fig.3b; Supplementary Fig.3b). We additionally investigated whether this approach could be used to control the growth of Pto DC3000 in planta. For this purpose, we pre-treated for one hour Arabidopsis Col-0 plants with total RNAs from IR-CFA6/HRPL plants and further dipinoculated them with Pto DC3000. We found that these RNA extracts triggered a decreased Pto DC3000 titer at 2 dpi, a phenotype that was comparable to the ones observed in IRCFA6/HRPL and Col-0 plants inoculated with the Pto $\Delta c f a 6$ strain (Fig.4a, 5c). By contrast, application of total RNAs from CV plants did not alter growth of Pto DC3000 in the same conditions (Fig.5c), supporting a specific effect of antibacterial RNAs in this process. To assess whether such RNA-based biocontrol approach could also be effective in cultivated 
plants, we repeated the same assay on tomato (Solanum lycopersicum), which is the natural host of Pto DC3000. Pre-treatment of WT tomato leaves for one hour with RNA extracts from IR-CFA6/HRPL plants led to compromised Pto DC3000-induced necrotic disease symptoms and to a reduction in bacterial content compared to leaves pre-treated with RNA extracts from CV plants (Fig.5d, 5e, 5f; Supplementary Fig.5a, 5b, 5c). Collectively, these data demonstrate that external application of plant antibacterial RNAs can trigger AGS and disease protection against Pto DC3000 in both Arabidopsis and tomato plants.

Next, we interrogated which RNA entities are responsible for AGS upon external application of antibacterial RNAs. We first introduced the $d c l 2-1$, $d c l 3-1$ and $d c l 4-2$ mutations in the IRCFA6/HRPL\#4 reference line. These resulting IR-CFA6/HRPL\#4 dcl234 plants revealed high accumulation of IR-CFA6/HRPL transcripts, despite probable transcriptional repression of the $35 \mathrm{~S}_{\text {pro }}$ :IR-CFA6/HRPL transgene due to the use of T-DNA insertional mutant carrying the 35S promoter (Fig.6a) ${ }^{41,42}$. Furthermore, anti-cfa6 and anti- $h r p L$ siRNAs were undetectable in those plants (Fig.6b), which is consistent with a role of DCL2, DCL3 and DCL4 in the processing of IR-CFA6/HRPL. We subsequently extracted total RNAs from these plants, incubated them with Pto DC3000 cells, and further monitored $c f a 6$ and $h r p L$ mRNA levels by RT-qPCR analysis. Using this in vitro assay, we found that RNA extracts from IRCFA6/HRPL\#4 dcl234 plants were no longer able to trigger down-regulation of $c f a 6$ and $h r p L$ mRNAs (Fig.6c), despite high accumulation of CFA6/HRPL dsRNA precursor transcript (Fig.6a). By contrast, RNA extracts from the IR-CFA6/HRPL\#4 plants, which contain high levels of anti-cfa6 and anti-hrpL siRNAs (Fig.2c, 6b), triggered reduced accumulation of these bacterial mRNAs (Fig.5a, 6c).

We next decided to analyze the antibacterial activity of the above RNA extracts by monitoring their effects on stomatal reopening of Col-0-infected plants. A prerequisite for this assay was to verify that Col-0 leaf sections would not trigger the processing of $C F A 6 / H R P L$ 
dsRNAs, and thus the production of anti-cfa6 and anti-hrpL siRNAs, upon incubation with RNA extracts from IR-CFA6/HRPL\#4 dcl234 plants, which was validated by northern blot analysis (Fig.6d, Supplementary Fig.6b). Interestingly, when we further monitored stomatal aperture in Col-0-infected leaf tissues pre-treated for one hour with these RNA entities, we found that Pto DC3000 triggered normal stomata reopening, thereby mimicking the effect observed with control RNAs from Col-0 or dcl234 plants (Fig.6e; Supplementary Fig.6c). By contrast, RNA extracts from IR-CFA6/HRPL\#4 plants, which contain abundant anti-cfa6 and anti-hrpL siRNAs, triggered full suppression of stomatal reopening in the same conditions (Fig.6d, 6e). Collectively, these data indicate that CFA6/HRPL long dsRNAs are neither involved in AGS nor in pathogenesis reduction. They rather suggest that corresponding siRNAs are likely the RNA entities responsible for these phenotypes. To verify this assumption, we further purified sRNA species from IR-CFA6/HRPL plant total RNAs using a glass fiber filter-based method (Supplementary Fig.7a, 7b), and subjected them to stomatal reopening assay. We found that these sRNA species suppressed Pto DC3000-triggered stomatal reopening, to the same extent as IR-CFA6/HRPL plant total RNA extracts (Fig.6f; Supplementary Fig.7c). By contrast, long RNA species, which were not filtered through the above columns, were inactive (Fig.6f; Supplementary Fig.7c), further supporting that CFA6/HRPL long dsRNAs are not involved in this response. We have then synthesized CFA6/HRPL long dsRNAs and cognate double-stranded siRNAs in vitro to further test their antibacterial activity (Fig.6g). The in vitro-synthesized CFA6/HRPL long dsRNAs did not alter the ability of Pto DC3000 to reopen stomata, nor did CYP51 long dsRNAs used as controls (Fig.6h; Supplementary Fig.7d). By contrast, in vitro-synthesized anti-cfa6 and antihrpL siRNAs fully suppressed stomatal reopening (Fig.6h; Supplementary Fig.7d), and accordingly, triggered silencing of $c f a 6$ and $h r p L$ genes (Fig.6i). Altogether, these data 
provide evidence that siRNAs directed against $c f a 6$ and $h r p L$ genes are critical for AGS and pathogenesis reduction, while cognate long dsRNAs are ineffective for both processes.

Although the above findings indicate that siRNAs can trigger AGS and antibacterial activity, they do not firmly demonstrate that these RNA entities are causal for these phenomena. To address this, we decided to generate recombinant bacteria expressing a siRNA-resilient version of the $h r p L$ gene. To this end, we complemented the Pto $\Delta h r p L$ mutant with either a WT hrpL transgene or a mutated version that contains as many silent mutations as possible in the siRNA targeted region, which were predicted to alter the binding of siRNAs with the hrpL mRNA but to produce the same protein sequence (Fig.7a, 7b, Supplementary Table3). Both transgenes were expressed under the constitutive neomycin phosphotransferase II (NPTII) promoter (Fig.7c). The resulting recombinant bacteria, referred to as Pto $\Delta h r p L \mathrm{WT} h r p L$ and Pto $\Delta h r p L$ mut $h r p L$, were found to restore the ability of the Pto $\Delta h r p L$ strain to reopen stomata when inoculated on Col-0 leaf sections (Fig.7f; Supplementary Fig.9a, 9b), indicating that both transgenes are functional in a stomatal reopening assay. We further assessed the sensitivity of each recombinant bacterium to AGS. For this purpose, we incubated Pto $\Delta h r p L$ WT hrpL and Pto $\Delta h r p L$ mut hrpL strains with total RNA extracts from CV and IRCFA6/HRPL\#4 plants and further monitored hrpL transgene mRNA levels by RT-qPCR analysis. We found a significant decrease in the accumulation of $h r p L$ mRNAs expressed from the Pto $\Delta h r p L$ WT $h r p L$ strain, which was not detected upon treatment with RNA extracts from CV plants (Fig.7d). These data indicate that the WT hrpL transgene expressed from the Pto $\Delta h r p L$ WT $h r p L$ strain is sensitive to AGS despite its constitutive expression driven by the NPTII promoter. By contrast, the accumulation of $h r p L$ mRNAs expressed from the Pto $\Delta$ hrpL mut hrpL strain was unaltered in response to RNA extracts from IRCFA6/HRPL\#4 plants (Fig.7d), indicating that siRNAs no longer exert their AGS effect 
towards this recombinant bacterium. Collectively, these findings demonstrate that anti-hrpL siRNAs are causal for silencing of the hrpL gene in Pto DC3000 cells.

We finally investigated the responsiveness of each recombinant bacterial strain to siRNAdirected pathogenesis reduction by exploiting the Pto DC3000-induced stomatal reopening assay. To assess the specific effect of siRNAs towards suppression of $h r p L$-mediated stomatal reopening function, we cloned an IR-HRPL inverted repeat targeting the same $h r p L$ sequence region than the one targeted by the IR-CFA6/HRPL hairpin, and further validated its capacity to produce anti-hrpL siRNAs upon Agrobacterium-mediated transient expression in Nicotiana benthamiana leaves (Fig.7e). N. benthamiana total RNAs containing anti-hrpL siRNAs were found to fully suppress the ability of Pto DC3000 to reopen stomata (Fig.7f, Supplementary Fig.8). Importantly, similar results were obtained when $N$. benthamiana RNA extracts containing anti-hrpL siRNAs were incubated with the Pto $\Delta h r p L$ WT $h r p L$ strain (Fig.7f; Supplementary Fig.9a, 9b), supporting a sensitivity of this bacterial strain to siRNA action. By contrast, the Pto $\Delta h r p L$ mut $h r p L$ strain was fully competent in reopening stomata (Fig.7f; Supplementary Fig.9a, 9b), indicating that anti-hrpL siRNAs no longer exert their antibacterial effects towards this recombinant bacterium. Similar results were also observed in Arabidopsis stable IR-HRPL transgenic lines overexpressing anti- $h r p L$ siRNAs (Fig.7g, 7h; Supplementary Fig.9c). This indicates that the suppression of stomatal reopening phenotype is not due to potential off-target effects of these host-encoded siRNAs but rather caused by their targeting effects over the $h r p L$ transcript sequence. Altogether, these data provide sound evidence that anti-hrpL siRNAs are causal for the suppression of $h r p L$-mediated stomatal reopening function. They also further validate a novel role of $h r p L$ in bacterial-induced stomatal reopening, indicating that AGS can be employed as a tool to characterize bacterial gene function. 


\section{Discussion}

We show here that Arabidopsis-encoded siRNAs trigger the silencing of Pto DC3000 virulence-associated genes, resulting in the dampening of pathogenesis. In particular, we found that anti-cfa6 and/or anti-hrpL siRNAs fully suppressed Pto DC3000-induced stomatal reopening, a virulence response that is dependent on the production of COR by epiphytic bacterial cells ${ }^{46}$. Our data therefore indicate that antibacterial siRNAs can act at the preinvasive stage of the infection, presumably by preventing COR biosynthesis in Pto DC3000 cells that come in contact with the leaf surface. In addition, we found that AGS was capable of reducing the ability of Pto DC3000 to mount water-soaking symptoms, to multiply in the leaf apoplast and to propagate in the leaf vasculature of Arabidopsis. Therefore, siRNAs additionally act at a post-invasive stage of the infection by targeting endophytic bacterial cells present in the apoplast and in xylem vessels. These observations imply that siRNAs must be externalized from plant cells towards the leaf surface, the apoplast and xylem vessels in order to reach epiphytic and endophytic bacterial populations. One siRNA trafficking mechanism might involve plant extracellular vesicles (EVs), whose secretion is enhanced during antibacterial defense and which contain diverse species of sRNAs $^{43}{ }^{44}$. Such a hypothesis would be consistent with recent findings showing that EVs ensure the movement of siRNAs from plant cells to fungal or oomycete cells ${ }^{7,11}$. It would also be congruent with a recent report showing that plant-derived exosome-like nanoparticles ingested by mice deliver plant miRNAs into specific commensal bacteria to reprogram their gene expression ${ }^{45}$. In accordance with these studies, we found that EVs isolated from the Arabidopsis IRCFA6/HRPL\#4 leaf apoplast, which were in a size range between 50 to $300 \mathrm{~nm}^{44}$ (Fig.8a; Supplementary Fig.10a), were fully competent in suppressing stomatal reopening, as observed with the apoplastic fluid from these transgenic plants (Fig.8b; Supplementary Fig.10b, 10d, 
10f). This suggests that apoplastic EVs may, at least in part, contribute to the trafficking of sRNAs from plant cells towards Pto DC3000 cells.

We additionally showed that incubating RNA extracts containing antibacterial siRNAs with Pto DC3000 cells was sufficient to trigger AGS. However, corresponding dsRNAs were ineffective for this process, suggesting that the latter RNA entities are either not taken-up by, or not properly processed in, Pto DC3000 cells. This is a major distinction from environmental RNAi in C. elegans and plant herbivores, which specifically relies on long dsRNAs $^{37-40,46}$, or in filamentous pathogens, which is triggered by both dsRNAs and siRNAs ${ }^{15,10}$. Instead, we found that plant siRNA species were causal for environmental RNAi in Pto DC3000, and this regulatory process was even, intriguingly, recapitulated in the presence of in vitro-synthesized siRNA duplexes. These data imply that Pto DC3000 must additionally be capable of taking-up - passively and/or actively- free sRNAs, despite the presence of a cell wall and an intricate double membrane structure. The data also suggest that Pto DC3000 must have evolved a machinery to take charge of the internalized sRNAs and direct gene silencing in bacterial cells. It will thus be appealing to identify such prokaryotic factors and to elucidate the principles of sRNA target recognition and mode of action in bacterial cells. Investigating whether our findings also apply to endogenous plant sRNAs, and assessing their possible implications in the regulation of the transcriptome, community composition and genome evolution of plant-associated bacteria will represent exciting directions for future studies. 


\section{Material and Methods}

\section{Plasmid construction}

The IR-CFA6/HRPL construct is composed of 250 bp regions of Pto DC3000 genes, cfa6 (1$250 \mathrm{nt})$ and $\mathrm{hrpl}(99-348 \mathrm{nt})$, aligned in sense and antisense directions with the intron of the petunia chalcone synthase gene $(C H S A)$ in between. The control vector $(\mathrm{CV})$ construct IRCYP51 is composed of described conserved regions from $F$. graminearum CYP51A, CYP51B and $C Y P 51 C^{13}$ and the IR-LUXA/LUXB is composed regions from $l u x A$ and $l u x B$ genes of the $\operatorname{lu} C D A B E$ operon and they are aligned in sense and antisense directions with the same intron sequence as described above. The IR-CFA6/HRPL, IR-CYP51 and IR- $L U X A / L U X B$ constructs containing EcoRI and SalI sites at both extremities were synthesized by GenScript ${ }^{\circledR}$ and inserted by restriction enzymatic digestion into a modified pDON221-P5-P2 vector carrying additional EcoRI and SalI sites to facilitate the insertion of these long-inverted repeats. The plasmids containing the $35 \mathrm{~S}_{\text {pro }}$ :IR-CFA6/HRPL and $35 \mathrm{~S}_{\text {pro }}$ :IR-CYP51 were obtained by a double recombination between pDON221-P5-P2 carrying the inverted repeat sequences and pDON221-P1-P5r carrying the Cauliflower Mosaic Virus (CaMV) 35S promoter sequence, in the the pB7WG Gateway destination vector using LR clonase plus (Life Technologies). These plasmids were then introduced into the Agrobacterium C58C1 strain. The IR-HRPL construct, which is composed of the same 250 bp region of $h r p L$ as in the IR-CFA6/HRPL construct, was recombined by using GreenGate technology ${ }^{47}$ to constitute the plasmid $35 \mathrm{~S}_{\text {pro }}: \mathrm{IR}-H R P L$, which was then transformed in the Agrobacterium C58C1 strain. To generate the WT hrpL and the mut $h r p L$ plasmids, the wild type $h r p L$ sequence was amplified from the genomic DNA isolated from Pto DC3000, while the mutant $h r p L$ sequence was amplified from a mutated sequence synthesized by GenScript ${ }^{\circledR}$. These two sequences were further cloned into pDON207 vector using BP clonase (Life Technologies) and then introduced by recombination using LR clonase (Life Technologies) into the pBS0046 destination vector, which carries a 
bioRxiv preprint first posted online Dec. 3, 2019; doi: http://dx.doi.org/10.1101/863902. The copyright holder for this preprint

constitutive NPTII promoter. Specific primers used for the purpose of cloning are listed in Supplementary table 4.

\section{Plant material and growth conditions}

Stable transgenic lines expressing IR- $L U X A / L U X B$, IR-CFA6/HRPL, IR-CYP51 (CV) and IRHRPL constructs were generated by transforming Arabidopsis (accession Col-0) plants using Agrobacterium mediated-floral dip method ${ }^{48}$. Two independent Arabidopsis T2 transgenic lines of IR-LUXA/LUXB, \#18 and \#20; three independent Arabidopsis T4 transgenic lines of IR-CFA6/HRPL, \#4, \#5 and \#10; two independent Arabidopsis T2 transgenic lines of IRHRPL, \#1 and \#4 and one reference Arabidopsis T4 transgenic line for IR-CYP51 \#2 (CV) were generated and used in our experiments. For genetic analysis, $d c l 2-1 d c l 3-1 d c l 4-2$ (dcl234) triple mutant was crossed with the reference line IR-CFA6/HRPL\#4 and the F3 plants were genotyped to select homozygous $d c l 234$ mutant containing the IR-CFA6/HRPL transgene. Sterilized seeds of Arabidopsis Col-0 and the selected homozygous transgenic lines were first grown for $12-14$ days at $22^{\circ} \mathrm{C}$ on plates containing $1 / 2 \times$ MS medium (Duchefa), $1 \%$ sucrose and $0.8 \%$ agar (with or without antibiotic selection) in $8 \mathrm{~h}$ photoperiod. Seedlings were then pricked out to soil pots and grown in environmentally controlled conditions at $22^{\circ} \mathrm{C} / 19^{\circ} \mathrm{C}$ (day/night) with an $8 \mathrm{~h}$ photoperiod under light intensity of $100 \mu \mathrm{E} / \mathrm{m}^{2} / \mathrm{s}$. Four- to five-week-old plants were used for all the experiments. Seeds of tomato (Solanum lycopersicum 'Moneymaker') and Nicotiana benthamiana were directly germinated on soil pots and grown in environmentally controlled conditions at $22{ }^{\circ} \mathrm{C} / 19^{\circ} \mathrm{C}$ (day/night) with a $16 \mathrm{~h}$ photoperiod under light intensity of $100 \mu \mathrm{E} / \mathrm{m}^{2} / \mathrm{s}$. Four- to five-week old plants were used for all the experiments. 


\section{Bacterial strains}

The Pto 4 cfa6-GFP (Pto DC3118-GFP) strain is a gift from S. Y. He, while the PtodhrpL strain is a gift from C. Ramos ${ }^{49}$. The Pto $\triangle$ hrpL strain expressing the GFP reporter gene was generated by transformation with the GFP-pPNpt Green plasmid by electroporation and then plated on NYGB medium (5 $\mathrm{g} \mathrm{L}^{-1}$ bactopeptone, $3 \mathrm{~g} \mathrm{~L}^{-1}$ yeast extract, $20 \mathrm{ml} \mathrm{L}^{-1}$ glycerol) containing gentamycin $\left(1 \mu \mathrm{g} \mathrm{mL}{ }^{-1}\right)$ for selection at $28^{\circ} \mathrm{C}$. To generate the Pto DC3000 WT hrpL and mut hrpL strains, the Pto $4 h r p L$ strain was transformed with the plasmids $N P T I I_{\text {pro: }}:$ TT-hrpL and $N P T I I_{\text {pro }}:$ mut- $h r p L$, respectively, by electroporation and then plated in NYGB medium with gentamycin $\left(1 \mu \mathrm{g} \mathrm{mL} \mathrm{m}^{-1}\right)$ at $28^{\circ} \mathrm{C}$ for two days. The colonies containing the plasmid were selected by PCR using specific primers to hrpL gene. The lux-tagged Pto DC3000 strain has been previously described ${ }^{21}$.

\section{Bacterial infection assays in plants}

(a) Bacterial dipping assay: Three hours after the beginning of the night cycle in growth chamber, three plants per condition were dip-inoculated with Pto DC3000 at $5 \times 10^{7} \mathrm{cfu} \mathrm{ml}^{-1}$ supplemented with $0.02 \%$ Silwet L-77 (Lehle seeds). Plants were then immediately placed in chambers with high humidity. Water-soaking symptoms were observed 24 hours postinfection and pictures of representative leaves were taken. Two days post-inoculation, bacterial titer was measured for individual infected leaves $(n=8)$. To quantify bacterial transcripts in infected plants, pool of infected leaves from three plants was collected three days post-inoculation.

(b) Wound-inoculation assay: Bacterial propagation in the mid-veins was assessed as described previously ${ }^{34}$. Around 15 leaves from three plants per condition were inoculated with a toothpick dipped in GFP-tagged bacteria at a concentration of $5 \times 10^{6} \mathrm{cfu} \mathrm{ml}^{-1}$ and then the plants were covered for 3 days. Bacterial propagation was then analyzed by monitoring GFP 
bioRxiv preprint first posted online Dec. 3, 2019; doi: http://dx.doi.org/10.1101/863902. The copyright holder for this preprint

signal under a UV light using an Olympus MV $10 \times$ Macrozoom with a GFP filter and representative pictures of bacterial propagation were taken with a CCD camera AxioCam Mrc Zeiss.

(c) Plant protection assay: Prior to bacterial infection, four leaves of three Col-0 plants were dipped with mock solution (water) or $20 \mathrm{ng} \mu \mathrm{l}^{-1}$ of specific total RNAs, both supplemented with $0.02 \%$ of Silwet L-77. After one hour, leaves were dip-inoculated with Pto DC3000 WT or Pto $\triangle$ cfa6 at $5 \times 10^{7} \mathrm{cfu} \mathrm{ml}^{-1}$ with $0.02 \%$ of Silwet L-77. Bacterial titers were monitored two days post-inoculation, as specified earlier. Similar assay was performed using tomato plants but with a GFP-tagged Pto DC3000. Representative pictures of bacterial disease symptoms observed at 3 dpi were depicted.

(d) Bacterial luminescence quantification: Three plants per condition were syringeinfiltrated with the lux-tagged Pto DC3000 strain at $10^{6} \mathrm{cfu} \mathrm{ml}^{-1}$. Plants were placed in a chamber with high humidity to facilitate proper infection. After 24 hours, leaf discs were prepared and placed in individual wells of a 96 well plate to quantify the luminescence using Berthold Centro LB 960 Microplate Luminometer. Four leaves per plant were taken into consideration. Leaf discs from individual leaves were collected and pooled to monitor bacterial titers as described in (a).

\section{Tomato infection quantification}

(a) GFP loci quantification: Tomato leaves infected with Pto DC3000-GFP strain were analyzed under a UV light using an Olympus MV 10x Macrozoom with a GFP filter and pictures were taken with a CCD camera AxioCam Mrc Zeiss. Number of GFP loci was quantified with ImageJ software for at least 10 pictures per condition. Individual leaves were collected to extract genomic DNA. 
bioRxiv preprint first posted online Dec. 3, 2019; doi: http://dx.doi.org/10.1101/863902. The copyright holder for this preprint

(b) Bacterial genomic DNA quantification: To quantify bacterial infection in the infected tomato plants, the amount of bacterial genomic DNA (gDNA) was measured relative to plant gDNA $^{50}$. Genomic DNA was isolated from tomato leaf samples infected with Pto DC3000GFP using the DNeasy plant mini kit (QIAGEN, Germany) according to the manufacturer's instructions. Using $1 \mathrm{ng}$ of gDNA, qPCR was performed using Takyon SYBR Green Supermix (Eurogentec ${ }^{\circledR}$ ) and GFP-specific primers. Amount of bacterial gDNA was normalized to that of tomato using Ubiquitin-specific primers. Primer sequences are listed in Supplementary table 4 .

\section{Agrobacterium-mediated transient expression of inverted repeats in N. benthamiana}

To express the IR-HRPL hairpin and the IR-CFA6/HRPL chimeric hairpin transiently, the $A$. tumefaciens $\mathrm{C} 58 \mathrm{C} 1$ strains carrying these plasmids were grown overnight in LB medium at $28^{\circ} \mathrm{C}$. Cells were resuspended in a solution containing $10 \mathrm{mM}$ MES, $\mathrm{pH} 5.6,10 \mathrm{mM} \mathrm{MgCl} 2$ and $200 \mu \mathrm{M}$ acetosyringone at a final concentration of $0.5 \mathrm{OD}_{600}$. Cultures were incubated in the dark at room temperature for 5-6 hours before Agrobacterium-mediated infiltration in 4week old $N$. benthamiana. After 3 days, leaves were harvested for total RNA extraction and molecular analyses.

\section{Stomatal aperture measurements}

Using intact leaves from three plants, sections were dissected and immersed in mock solution (water) or bacterial suspension at $10^{8} \mathrm{cfu} \mathrm{m}^{-1}$. After 3 hours, unpeeled leaf sections were stained with $10 \mu \mathrm{g} \mathrm{ml}^{-1}$ propidium iodide (Sigma) and abaxial surface was observed under SP5 laser scanning confocal microscope. The stomatal aperture (width relative to length) was measured using ImageJ software for at least 50-70 stomata per condition. For RNA and vesicle treatments, the leaf sections were incubated with total RNAs or APF and P40 fraction 
bioRxiv preprint first posted online Dec. 3, 2019; doi: http://dx.doi.org/10.1101/863902. The copyright holder for this preprint

extracted from specified genotypes for one hour before incubation with the bacteria. In specified experiments, $1 \mu \mathrm{M}$ of Coronatine (COR; Sigma) was supplemented to the bacterial suspension.

\section{In vitro AGS assay}

To assess whether the transcripts of Pto DC3000 cfa6 and $h r p L$ can be directly targeted by the dsRNA and/or the siRNAs generated by the hairpin IR-CFA6/HRPL, $2 \mathrm{ml}$ cultures of Pto

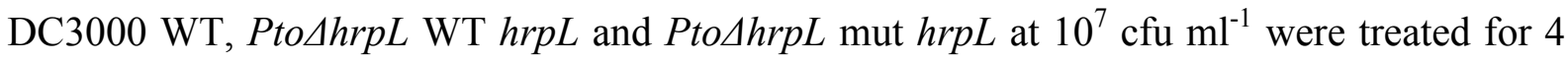
and/or 8 hours, with $20 \mathrm{ng} \mu \mathrm{l}^{-1}$ of specified total RNA extracted from CV or IRCFA6/HRPL\#4 transgenic plants. For each condition, bacteria were harvested and processed for molecular analyses.

\section{Quantitative RT-PCR Analyses}

To monitor plant-encoded transcripts, total RNA was extracted from plant samples using Nucleospin RNA plant kit (Macherey Nagel). $0.5 \mu \mathrm{g}$ of DNase-treated RNA was reverse transcribed using qScript cDNA Supermix (Quanta Biosciences $\left.{ }^{\circledR}\right)$. cDNA was then amplified by real time PCR reactions using Takyon SYBR Green Supermix (Eurogentec ${ }^{\circledR}$ ) and genespecific primers. Expression was normalized to that of Arabidopsis Ubiquitin. To monitor bacterial transcripts, total RNA was extracted from bacteria-infected plant samples or from in vitro treated bacteria using Nucleospin RNA kit. After DNAse treatment, $250 \mathrm{ng}$ of total RNA was reverse transcribed using random hexamer primers and qScript Flex cDNA kit (Quanta Biosciences ${ }^{\circledR}$ ) and then amplified by real time PCR reaction using gene-specific primers. Expression was normalized to that of gyrA. Real time PCR was performed in 384-well optical reaction plates heated at $95^{\circ} \mathrm{C}$ for $10 \mathrm{~min}$, followed by 45 cycles of denaturation at $95^{\circ} \mathrm{C}$ for $15 \mathrm{~s}$, annealing at $60^{\circ} \mathrm{C}$ for $20 \mathrm{~s}$, and elongation at $72^{\circ} \mathrm{C}$ for $40 \mathrm{~s}$. A melting curve was 
bioRxiv preprint first posted online Dec. 3, 2019; doi: http://dx.doi.org/10.1101/863902. The copyright holder for this preprint

performed at the end of the amplification by steps of $1^{\circ} \mathrm{C}$ (from $95^{\circ} \mathrm{C}$ to $50^{\circ} \mathrm{C}$ ). Primer sequences are listed in Supplementary table 3.

\section{RNA Gel Blot Analyses}

Accumulation of low molecular weight RNAs was assessed by Northern blot analysis as previously described ${ }^{31}$. Total RNA was extracted using TriZOL reagent and stabilized in 50\% formamide and $30 \mu \mathrm{g}$ of total RNAs were used. To generate specific ${ }^{32} \mathrm{P}$-radiolabelled probes, regions of $150 \mathrm{bp}$ to $300 \mathrm{bp}$ were amplified from the plasmids using gene specific primers (Supplementary Table 4) and the amplicons were labeled by random priming (Prime-a-Gene ${ }^{\circledR}$ Labeling System, Promega). U6 was monitored as an equal loading control.

\section{Separation of long and small RNA fractions}

From $100 \mu \mathrm{g}$ of TriZOL-extracted total RNA, long and small RNA fractions were separated using the mirVana miRNA isolation kit (Ambion $\left.{ }^{\circledR}\right)$ according to the manufacturer's instructions. The long and small RNA fractions were visualized using agarose gel electrophoresis and further analyzed using Bioanalyzer 2100 (Agilent Technologies).

\section{In vitro synthesis of Inverted Repeat (IR) RNAs}

In vitro synthesized RNAs were generated following the instruction of the MEGAscript ${ }^{\circledR}$ RNAi Kit (Life Technologies, Carlsbad, CA). Templates were amplified by PCR using gene specific primers containing the $\mathrm{T} 7$ promoter. PCR amplification was done in two steps with two different annealing temperatures to increase the specificity of primers annealing. After the amplification step, PCR products were purified by gel extraction using the PCR Clean-up kit (Macherey-Nagel) to eliminate any unspecific PCR products. To produce dsRNAs, the purified PCR products were used as templates for in vitro transcription performed according 
bioRxiv preprint first posted online Dec. 3, 2019; doi: http://dx.doi.org/10.1101/863902. The copyright holder for this preprint

to the MEGAscript RNAi Kit instructions (Life Technologies). After purification with the filter catridges, the corresponding dsRNAs were processed in 18-25 nt siRNAs by ShortCut ( RNase III (NEB, Ipswich, MA) for $40 \mathrm{~min}$ at $37^{\circ} \mathrm{C}$. siRNAs were then specifically purified by using the mirVana ${ }^{\mathrm{TM}}$ miRNA Isolation Kit (Life Technologies, Carlsbad, CA). Each step of this process was followed by gel electrophoresis (TAE 1X, 1\% agarose gel for DNA amplification and 2\% agarose gel for RNAs) to verify the quality of RNAs.

\section{Apoplastic Fluid (APF) and Extracellular Vesicles (EVs) extraction}

Extraction was done as previously described ${ }^{17}$. Briefly, sixty leaves of 5 week-old CV or IRCFA6/HRPL plants were syringe-infiltrated with vesicle isolation buffer (20 mM MES, $2 \mathrm{mM}$ $\mathrm{CaCl} 2,0.01 \mathrm{M} \mathrm{NaCl}, \mathrm{pH}$ 6.0). Leaves were then placed inside a $20 \mathrm{ml}$ needleless syringe that was inserted in a $50 \mathrm{ml}$ Falcon and centrifuged at $900 \mathrm{~g}$ for 15 minutes at $4{ }^{\circ} \mathrm{C}$. The apoplastic fluid (APF) was collected and, to get rid of any cell debris, filtered through $0.45 \mu \mathrm{m}$ filter before to be centrifuged at $10,000 \mathrm{~g}$ for 30 minutes at $4^{\circ} \mathrm{C}$. The APF was subjected to an ultracentrifugation step at $40,000 \mathrm{~g}$ for $60 \mathrm{~min}$ at $4^{\circ} \mathrm{C}$ to pellet $\mathrm{EV}$ fraction (P40) that was washed with vesicle isolation buffer and pelleted again. The pellet was further resuspended in $2 \mathrm{ml}$ of $20 \mu \mathrm{M}$ Tris buffer $\mathrm{pH}=7.5$ and stored at $4{ }^{\circ} \mathrm{C}$. The size and concentration of EVs from both the APF and P40 fractions were analyzed using a LM10 Nanoparticle Tracking Analysis device (Malvern) $)^{51}$.

\section{Bioinformatic analysis}

Small RNA libraries were constructed and sequenced from four- to five-week old leaves of IR-CFA6/HRPL\#4. Raw reads have been deposited at the NCBI SRA under Bioproject (PRJNA587213). Libraries were mapped against the Arabidopsis thaliana genome (v TAIR10.1 GCF_000001735.4) and the IR-CFA6/HRPL sequence using ShortStack (default 
parameters $)^{52}$. Coverage of mapped loci was obtained with the Genomic Alignments package in $\mathrm{R}^{53}$. Unique reads mapping to $c f a 6$ and $h r p L$ in both replicates were extracted and quantified from ShortStack mapping results using samtools ${ }^{54}$. Each unique read was aligned against annotated Arabidopsis and Pto DC3000 coding sequences (CDS) using BLAST ${ }^{55}$ (-evalue 10, -word_size 4, -ungapped, -reward 1, -penalty -1), and the top target (with lowest evalue) was kept for each read in each target set. Binding free energy was calculated for each read/target pair using RNAup (-interaction pairwise) ${ }^{56}$.

\section{References}

1. Baulcombe, D.C. VIGS, HIGS and FIGS: small RNA silencing in the interactions of viruses or filamentous organisms with their plant hosts. Curr Opin Plant Biol 26, 141-6 (2015).

2. Pumplin, N. \& Voinnet, O. RNA silencing suppression by plant pathogens: defence, counter-defence and counter-counter-defence. Nat Rev Microbiol 11, 745-60 (2013).

3. Staiger, D., Korneli, C., Lummer, M. \& Navarro, L. Emerging role for RNA-based regulation in plant immunity. New Phytol 197, 394-404 (2013).

4. Bologna, N.G. \& Voinnet, O. The diversity, biogenesis, and activities of endogenous silencing small RNAs in Arabidopsis. Annu Rev Plant Biol 65, 473-503 (2014).

5. Liu, L. \& Chen, X. Intercellular and systemic trafficking of RNAs in plants. Nat Plants $\mathbf{4}$, 869-878 (2018).

6. Melnyk, C.W., Molnar, A. \& Baulcombe, D.C. Intercellular and systemic movement of RNA silencing signals. EMBO J 30, 3553-63 (2011).

7. Cai, Q. et al. Plants send small RNAs in extracellular vesicles to fungal pathogen to silence virulence genes. Science 360, 1126-1129 (2018).

8. Guo, Z., Li, Y. \& Ding, S.W. Small RNA-based antimicrobial immunity. Nat Rev Immunol 19, 31-44 (2019).

9. Zhang, T. et al. Cotton plants export microRNAs to inhibit virulence gene expression in a fungal pathogen. Nat Plants 2, 16153 (2016). 
bioRxiv preprint first posted online Dec. 3, 2019; doi: http://dx.doi.org/10.1101/863902. The copyright holder for this preprint

10. Wang, M. et al. Bidirectional cross-kingdom RNAi and fungal uptake of external RNAs confer plant protection. Nat Plants 2, 16151 (2016).

11. Hou, Y. et al. A Phytophthora Effector Suppresses Trans-Kingdom RNAi to Promote Disease Susceptibility. Cell Host Microbe 25, 153-165 e5 (2019).

12. Weiberg, A. et al. Fungal small RNAs suppress plant immunity by hijacking host RNA interference pathways. Science 342, 118-23 (2013).

13. Ren, B., Wang, X., Duan, J. \& Ma, J. Rhizobial tRNA-derived small RNAs are signal molecules regulating plant nodulation. Science 365, 919-922 (2019).

14. Koch, A. et al. Host-induced gene silencing of cytochrome P450 lanosterol C14alphademethylase-encoding genes confers strong resistance to Fusarium species. Proc Natl Acad Sci U S A 110, 19324-9 (2013).

15. Koch, A. et al. An RNAi-Based Control of Fusarium graminearum Infections Through Spraying of Long dsRNAs Involves a Plant Passage and Is Controlled by the Fungal Silencing Machinery. PLoS Pathog 12, e1005901 (2016).

16. Wang, M. \& Jin, H. Spray-Induced Gene Silencing: a Powerful Innovative Strategy for Crop Protection. Trends Microbiol 25, 4-6 (2017).

17. Wang, M., Thomas, N. \& Jin, H. Cross-kingdom RNA trafficking and environmental RNAi for powerful innovative pre- and post-harvest plant protection. Curr Opin Plant Biol 38, 133-141 (2017).

18. Mansfield, J. et al. Top 10 plant pathogenic bacteria in molecular plant pathology. Mol Plant Pathol 13, 614-29 (2012).

19. Preston, G.M. Pseudomonas syringae pv. tomato: the right pathogen, of the right plant, at the right time. Mol Plant Pathol 1, 263-75 (2000).

20. Fan et al. High-throughput quantitative luminescence assay of the growth in planta of Pseudomonas syringae chromosomal tagged with Photorhabdus luminescent luxCDABE. Plant Journal 53(2):393-9 (2008).

21. Meighen, E.A. Molecular biology of bacterial bioluminescence. Microbiol Rev 55, 123-42 (1991).

22. Xin, X.F., Kvitko, B. \& He, S.Y. Pseudomonas syringae: what it takes to be a pathogen. Nat Rev Microbiol 16, 316-328 (2018). 
bioRxiv preprint first posted online Dec. 3, 2019; doi: http://dx.doi.org/10.1101/863902. The copyright holder for this preprint

23. Bender, C.L., Alarcon-Chaidez, F. \& Gross, D.C. Pseudomonas syringae phytotoxins: mode of action, regulation, and biosynthesis by peptide and polyketide synthetases. Microbiol Mol Biol Rev 63, 266-92 (1999).

24. Bender, C. L., and Scholz-Schroeder, B. K. New insights into the biosynthesis, mode of action, and regulation of syringomycin, syringo- peptin and coronatine. The Pseudomonads. 125-158, ed. Kluwer Academic Press, Dordrecht (2004).

25. Jin, Q., Thilmony, R., Zwiesler-Vollick, J. \& He, S.Y. Type III protein secretion in Pseudomonas syringae. Microbes Infect 5, 301-10 (2003).

26. Brooks, D.M. et al. Identification and characterization of a well-defined series of coronatine biosynthetic mutants of Pseudomonas syringae pv. tomato DC3000. Mol Plant Microbe Interact 17, 162-74 (2004).

27. Sreedharan, A., Penaloza-Vazquez, A., Kunkel, B.N. \& Bender, C.L. CorR regulates multiple components of virulence in Pseudomonas syringae pv. tomato DC3000. Mol Plant Microbe Interact 19, 768-79 (2006).

28. Fouts, D.E. et al. Genomewide identification of Pseudomonas syringae pv. tomato DC3000 promoters controlled by the HrpL alternative sigma factor. Proc Natl Acad Sci U S A 99, 2275-80 (2002).

29. Melotto, M., Underwood, W. \& He, S.Y. Role of stomata in plant innate immunity and foliar bacterial diseases. Annu Rev Phytopathol 46, 101-22 (2008).

30. Melotto, M., Underwood, W., Koczan, J., Nomura, K. \& He, S.Y. Plant stomata function in innate immunity against bacterial invasion. Cell 126, 969-80 (2006).

31. Navarro, L., Jay, F., Nomura, K., He, S.Y. \& Voinnet, O. Suppression of the microRNA pathway by bacterial effector proteins. Science 321, 964-7 (2008).

32. Shivaprasad, P.V. et al. A microRNA superfamily regulates nucleotide binding siteleucine-rich repeats and other mRNAs. Plant Cell 24, 859-74 (2012).

33. Xin, X.F. et al. Bacteria establish an aqueous living space in plants crucial for virulence.

Nature 539, 524-529 (2016).

34. Yu, A. et al. Dynamics and biological relevance of DNA demethylation in Arabidopsis antibacterial defense. Proc Natl Acad Sci U S A 110, 2389-94 (2013). 
bioRxiv preprint first posted online Dec. 3, 2019; doi: http://dx.doi.org/10.1101/863902. The copyright holder for this preprint

35. Misas-Villamil, J.C., Kolodziejek, I. \& van der Hoorn, R.A. Pseudomonas syringae colonizes distant tissues in Nicotiana benthamiana through xylem vessels. Plant $J$ 67, 774-82 (2011).

36. Winston, W.M., Sutherlin, M., Wright, A.J., Feinberg, E.H. \& Hunter, C.P. Caenorhabditis elegans SID-2 is required for environmental RNA interference. Proc Natl Acad Sci U S A 104, 10565-70 (2007).

37. Whangbo, J.S. \& Hunter, C.P. Environmental RNA interference. Trends Genet 24, 297305 (2008).

38. McEwan, D.L., Weisman, A.S. \& Hunter, C.P. Uptake of extracellular double-stranded RNA by SID-2. Mol Cell 47, 746-54 (2012).

39. Feinberg, E.H. \& Hunter, C.P. Transport of dsRNA into cells by the transmembrane protein SID-1. Science 301, 1545-7 (2003).

40. Ivashuta, S. et al. Environmental RNAi in herbivorous insects. RNA 21, 840-50 (2015).

41. Daxinger, L. et al. Unexpected silencing effects from T-DNA tags in Arabidopsis. Trends Plant Sci 13, 4-6 (2008).

42. Mlotshwa, S. et al. Transcriptional silencing induced by Arabidopsis T-DNA mutants is associated with 35S promoter siRNAs and requires genes involved in siRNA-mediated chromatin silencing. Plant J 64, 699-704 (2010).

43. Baldrich, P. et al. Plant Extracellular Vesicles Contain Diverse Small RNA Species and Are Enriched in 10 to 17 Nucleotide "Tiny" RNAs. Plant Cell (2019).

44. Rutter, B.D. \& Innes, R.W. Extracellular Vesicles Isolated from the Leaf Apoplast Carry Stress-Response Proteins. Plant Physiol 173, 728-741 (2017).

45. Teng, Y. et al. Plant-Derived Exosomal MicroRNAs Shape the Gut Microbiota. Cell Host Microbe 24, 637-652 e8 (2018).

46. Bolognesi, R. et al. Characterizing the mechanism of action of double-stranded RNA activity against western corn rootworm (Diabrotica virgifera virgifera LeConte). PLoS One 7, e47534 (2012)

47. Lampropoulos, A. et al. GreenGate - a novel, versatile, and efficient cloning system for plant transgenesis. PLoS One 8, e83043 (2013). 
bioRxiv preprint first posted online Dec. 3, 2019; doi: http://dx.doi.org/10.1101/863902. The copyright holder for this preprint

48. Clough, S.J. \& Bent, A.F. Floral dip: a simplified method for Agrobacterium-mediated transformation of Arabidopsis thaliana. Plant J 16, 735-43 (1998).

49. Castillo-Lizardo, M.G. et al. Contribution of the non-effector members of the HrpL regulon, iaaL and matE, to the virulence of Pseudomonas syringae pv. tomato DC3000 in tomato plants. BMC Microbiol 15, 165 (2015).

50. Ross, A. \& Somssich, I.E. A DNA-based real-time PCR assay for robust growth quantification of the bacterial pathogen Pseudomonas syringae on Arabidopsis thaliana. Plant Methods 12, 48 (2016).

51. Kowal, J. et al. Proteomic comparison defines novel markers to characterize heterogeneous populations of extracellular vesicle subtypes. Proc Natl Acad Sci U S A 113, E968-77 (2016).

52. Axtell, M.J. ShortStack: comprehensive annotation and quantification of small RNA genes. $R N A$ 19, 740-51 (2013).

53. Lawrence, M. et al. Software for computing and annotating genomic ranges. PLoS Comput Biol 9, e1003118 (2013).

54. Li, H. A statistical framework for SNP calling, mutation discovery, association mapping and population genetical parameter estimation from sequencing data. Bioinformatics 27, 2987-93 (2011).

55. Altschul, S.F. et al. Gapped BLAST and PSI-BLAST: a new generation of protein database search programs. Nucleic Acids Res 25, 3389-402 (1997).

56. Lorenz, W.A. \& Clote, P. Computing the partition function for kinetically trapped RNA secondary structures. PLoS One 6, e16178 (2011).

\section{Acknowledgments}

We thank S.-H. He for the Pto DC3118-GFP strain, C. Ramos for the Pto DC3000 hrpL mutant strain, Lorena Martin-Jaular and Federico Cocozza from C. Théry Lab to provide help for the use of the particle tracker analyzer and members of the Navarro laboratory for critical reading of the manuscript. M. S-R., V.M. and L.N. received support by a European Research Council (ERC) “Silencing \& Immunity” granted to L.N. (281749). M S-R received additional 
bioRxiv preprint first posted online Dec. 3, 2019; doi: http://dx.doi.org/10.1101/863902. The copyright holder for this preprint

(which was not peer-reviewed) is the author/funder, who has granted bioRxiv a license to display the preprint in perpetuity.

All rights reserved. No reuse allowed without permission.

Ph.D. funding support under the program "Investissements d'Avenir" and implemented by ANR (ANR-10-LABX-54 MEMO LIFE, ANR-10-IDEX-0001-02PSL). O.T. and A.E.F. received support from the programmes "Jeune Entreprise Innovante" (JEI) and "Crédit Impôt Recherche" (CIR).

\section{Author contributions}

M. S-R., M.C., O.T. and A.R. performed the experiments, A.E.F. and V.M. generated some constructs, A.P-Q conducted bioinformatic analyses, M. S-R., O.T., and L.N. analyzed the data; M. S-R., O.T. and L.N. designed the experiments, M. S-R. and L.N. wrote the manuscript, L.N. conceived the overall study.

\section{Competing interests}

Two patents have been applied for (PCT/EP2019/072169, PCT/EP2019/072170) on the AGS technology on which M. S-R and L.N. are listed as inventors. 


\section{Figure legends}

Fig. 1. Stable expression of the IR-LUXA/LUXB inverted repeat transgene in Arabidopsis triggers silencing of Pto DC3000 luxA and $l u x B$ genes during infection, which is accompanied by a decrease in luminescence activity

a. Schematic representation of the $\operatorname{lux} C D A B E$ operon chromosomally integrated in the genome of Pto DC3000 ${ }^{20}$. The $250 \mathrm{bp}$ regions of luxA (1-250 nt; highlighted region in pink) and $\operatorname{luxB}(1-250 \mathrm{nt}$; highlighted region in violet) were used to generate the chimeric hairpin construct under the control of the constitutive $35 \mathrm{~S}$ promoter, hereby designated as $35 \mathrm{~S}_{\text {pro }}$ :IR$L U X A / L U X B$. b. Accumulation levels of anti-luxA and anti-luxB siRNAs in the two independent Arabidopsis transgenic lines expressing $35 \mathrm{~S}_{\text {pro }}: \mathrm{IR}-L U X A / L U X B$ were detected by low molecular weight northern blot analysis. U6 was used as a loading control. c. Luminescence activity of lux-tagged Pto DC3000 (Pto WT LUC) is decreased in the infected Arabidopsis transgenic lines carrying the $35 \mathrm{~S}_{\text {pro }}$ :IR- $L U X A / L U X B$ transgene compared to the infected Col-0 plants. Col-0 plants and IR-LUXA/LUXB \#18 and \#20 transgenic lines were syringe-infiltrated with Pto WT LUC at $10^{6} \mathrm{cfu} \mathrm{m}^{-1}$. The bacterial luminescence and titers were monitored at 1 day post-infection (dpi). Four leaves from three plants per condition and from three independent experiments (n) are considered for the analysis. The luminescence activity was estimated by calculating the ratio of bacterial luminescence recorded from individual leaf sample and the number of bacteria (data presented in d.) counted for the corresponding sample. d. The IR-LUXA/LUXB transgenic lines, \#18 and \#20, do not exhibit significantly reduced Pto WT LUC titer when compared to Col-0-infected plants. Bacterial titers were monitored for the same samples used in c. e. Accumulation level of both $\operatorname{luxA}$ and luxB mRNAs are significantly decreased on IR-LUXA/LUXB-infected plants compared to Col-0-infected plants. Using the same samples as described in c. and d., bacterial transcript levels of proC, $\operatorname{lux} A$ and $\operatorname{lux} B$ were monitored by quantitative RT-PCR analysis. These 
bioRxiv preprint first posted online Dec. 3, 2019; doi: http://dx.doi.org/10.1101/863902. The copyright holder for this preprint

mRNA levels were quantified relative to the levels of bacterial gyrA transcript. Error bars indicate the standard deviations of mRNA values obtained in three independent experiments. Statistically significant differences were assessed using ANOVA test (ns: p-value $>0.05 ; *$ : pvalue $<0.05, * *$ : p-value $<0.01, * * * *:$-value $<0.0001)$

Fig. 2. Stable expression of the IR-CFA6/HRPL inverted repeat transgene in Arabidopsis triggers silencing of Pto DC3000 cfa6 and $h r p L$ genes during infection

a. Schematic representation of the Pto DC3000 genes $c f a 6$ (in blue) and $h r p L$ (in green). The $250 \mathrm{bp}$ regions of $c f a 6(1-250 \mathrm{nt})$ and $h r p L$ (99-348 nt) were used to generate the chimeric hairpin construct under the control of the constitutive $35 \mathrm{~S}$ promoter. b. Representative pictures of five-week old Arabidopsis Col-0 plants and of independent homozygous transgenic plants expressing the $35 \mathrm{~S}_{\text {pro }}$ :IR-CYP51 (Control vector: CV) or the $35 \mathrm{~S}_{\text {pro }}$ :IR$C F A 6 / H R P L$ transgenes. c. Accumulation level of anti-cfa6 and anti-hrpL siRNAs for the Arabidopsis plants depicted in b. was detected by low molecular weight northern blot analysis. U6 was used as a loading control. d. Size distribution and abundance of 20-24 nt long sRNA reads from IR-CFA6/HRPL\#4 transgenic line that were mapped to the $c f a 6$ (upper panel) and $h r p L$ (lower panel) regions of the IR-CFA6/HRPL sequence. Data from two biological replicates (rep1 and rep2) are presented. e. The coverage of sRNA reads which is computed as total count of mapped reads across the IR-CFA6/HRPL inverted repeat is depicted and include both the plus and minus strand of the construct. f. Pto DC3000 hrpL mRNA accumulation is significantly decreased in the three independent IR-CFA6/HRPLinfected transgenic plants compared to Col-0- and CV-infected plants. Arabidopsis plants depicted in b. were dip-inoculated with $10^{8} \mathrm{cfu} \mathrm{ml}^{-1}$ Pto DC3000 WT strain and at 3 days post-infection (dpi), bacterial transcript levels of $p r o C$, cfa6 and $h r p L$ were monitored by quantitative RT-PCR analysis. These mRNA levels are quantified relative to the level of 
bioRxiv preprint first posted online Dec. 3, 2019; doi: http://dx.doi.org/10.1101/863902. The copyright holder for this preprint

bacterial gyrA transcripts. Error bars indicate the standard deviations of mRNA values obtained in three independent experiments. Statistically significant differences were assessed using ANOVA test (ns: p-value $>0.05 ; *$ : p-value $<0.05, \quad * *$ : p-value $<0.01, \quad * * *$ : pvalue $<0.001)$.

Fig. 3. Stable expression of the IR-CFA6/HRPL inverted repeat transgene in Arabidopsis suppresses Pto DC3000-induced stomatal reopening

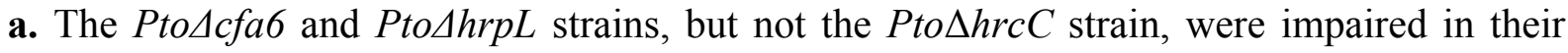
ability to reopen stomata and these phenotypes were rescued upon addition of exogenous COR. Sections of unpeeled leaves of Col-0 plants were incubated with mock solution (water) or $10^{8} \mathrm{cfu} \mathrm{ml}^{-1}$ of Pto DC3000 WT, $\Delta c f a 6, \Delta h r p L$ or $\Delta h r c C$ strains for 3 hours. Stomatal aperture was assessed by computing the ratio width over length using ImageJ software. b. Pto DC3000 WT no longer induced stomatal reopening in Arabidopsis transgenic lines overexpressing the IR-CFA6/HRPL inverted repeat transgene. Stomatal aperture measurement was conducted in Col-0 and IR-CFA6/HRPL\#4, \#5, \#10 transgenic lines infected with Pto WT strain as described in a. c. The Pto DC3000-induced stomatal reopening response was unaltered in CV compared to Col-0 plants. Stomatal aperture measurement was conducted in Col-0 and CV plants incubated with Pto WT strain as described in a. For all these experiments, $\mathrm{n}=$ number of stomata analyzed per condition and statistical significance was assessed using the ANOVA test (ns: p-value $>0.05 ; * * * *$ : p-value $<0.0001$ ). Similar results were obtained from at least three independent experiments (two other biological replicates are presented in Supplementary Fig.3). Pictures at the bottom of the graph depict the representative stomatal aperture phenotypes corresponding to the mean value for each condition/genotype. 
bioRxiv preprint first posted online Dec. 3, 2019; doi: http://dx.doi.org/10.1101/863902. The copyright holder for this preprint

Fig. 4. Stable expression of the IR-CFA6/HRPL inverted repeat transgene in Arabidopsis inhibits the ability of Pto DC3000 to multiply in the apoplast, to mount water-soaking symptoms and to spread in the leaf vasculature

a. Arabidopsis IR-CFA6/HRPL transgenic lines exhibit a significantly reduced Pto WT titer when compared to Col-0 and CV-infected plants. Col-0, CV and IR-CFA6/HRPL\#4, \#5 and \#10 plants were dip-inoculated with Pto WT-GFP and Col-0 plants were dip-inoculated with the Pto 4 cfa6-GFP strain. Bacterial titers were monitored at 2 days post-infection (dpi). Four leaves from three plants per condition and from three independent experiments (n) were considered for the comparative analysis. Statistical significance was assessed using the ANOVA test (ns: p-value $>0.05$; ****: p-value $<0.0001$ ). b. IR-CFA6/HRPL\#4, \#5 and \#10 transgenic plants exhibit reduced water-soaking symptoms in comparison to Col-0 and CV plants. Representative leaf pictures of water-soaking symptoms were taken 1 day after dipinoculation with Pto WT-GFP or PtoAcfa6-GFP strain. c. \& d. IR-CFA6/HRPL\#4, \#5 and \#10-infected plants exhibit reduced vascular spreading of Pto WT compared to Col-0- and $\mathrm{CV}$-infected plants. Plants were wound-inoculated in midveins with Pto WT-GFP and Col-0

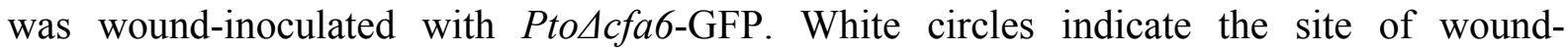
inoculation in the midvein. To index the spreading of bacteria from the inoculation sites, GFP fluorescence signal was observed under UV light and pictures were taken at 3 dpi. When the bacteria propagated away from any of the three inoculation sites, it was indexed as propagation with 4 corresponding to the highest propagation index. Pictures from three biological replicates were taken into consideration and one representative picture is depicted.

Fig. 5. Exogenously applied total RNA extracts from IR-CFA6/HRPL plants reduce Pto DC3000 pathogenesis in both Arabidopsis and tomato plants 
bioRxiv preprint first posted online Dec. 3, 2019; doi: http://dx.doi.org/10.1101/863902. The copyright holder for this preprint

a. In vitro AGS assay showing that total RNA extract from IR-CFA6/HRPL\#4 plants triggers silencing of both $c f a 6$ and hrpL genes. Pto WT cells were incubated in vitro for 4 and 8 hours with $20 \mathrm{ng} \mu \mathrm{l}^{-1}$ of total RNAs from CV or IR-CFA6/HRPL\#4 plants. Significant reduction of the bacterial transcripts $c f a 6$ and $h r p L$ was observed by RT-qPCR at both timepoints, while accumulation of $p r o C$ and $r p o B$ transcripts remained unaffected. gyr $A$ was used as an internal control to quantify the accumulation of bacterial transcripts. Error bars indicate the standard deviations of values from three independent experiments. b. The ability of Pto WT to reopen stomata was altered upon exogenous application of total RNA extracts from IR-CFA6/HRPL plants compared to CV plants. Col-0 leaves were treated for 1 hour with water or $20 \mathrm{ng} \mathrm{fl}^{-1}$ of total RNAs extracted from CV or IR-CFA6/HRPL\#4 plants and were incubated with Pto WT for 3 hours. Stomatal aperture was measured and analyzed as described in Fig.3a. Two other biological replicates are presented in Supplementary Fig.6a. c. Pretreatment with IRCFA6/HRPL\#4 total RNAs reduced the ability of Pto DC3000 to multiply in the apoplast of leaves when compared to pretreatment with CV total RNAs. Col-0 leaves were treated with $20 \mathrm{ng} \mu \mathrm{l}^{-1}$ of total RNAs from CV or IR-CFA6/HRPL\#4 plants for 1 hour, followed by dipinoculation with Pto WT. Bacterial titers were monitored at 2 dpi as in Fig.4a. The number of leaves (n) corresponds to collective values from three independent biological replicates. Statistically significant differences were assessed using ANOVA test (ns: p-value $>0.05 ; * *$ : p-value $<0.01 ; * * *$ : $\mathrm{p}$-value $<0.001)$. d. The tomato leaves treated with $\mathrm{CV}$ total RNAs displayed more necrotic symptoms as compared to the leaves treated with IR-CFA6/HRPL\#4 total RNAs. The experiment was conducted as in c. but using five-week-old tomato (Solanum lycopersicum, cultivar 'Moneymaker') plants. Representative pictures of infected leaves in the two conditions are depicted. e. A reduced number of Pto WT-GFP foci was observed in tomato leaves treated with total RNA extracts from IR-CFA6/HRPL\#4 vs CV plants. Infectedleaves were observed at 3 dpi under UV light to estimate the number of GFP loci. Left panel: 
Dot plot representing the number of GFP loci analyzed using Image J software from 3-4 different leaves per condition with at least 4 pictures per leaf. The values used for the analysis are from two different independent experiments. Right panel: Representative picture of the tomato leaves described in d. f. Pto WT-GFP DNA content is decreased in tomato leaves treated with total RNA extracts from IR-CFA6/HRPL\#4 vs CV plants. The level of bacterial DNA content was analyzed by qPCR using tomato Ubiquitin gene as a control. For e and f, student's t-test was performed for the comparative analysis (ns: p-value $>0.05$; **: pvalue $<0.01 ; * * *$ : p-value $<0.001 ; * * * *$ : p-value $<0.0001)$. Results of another biological replicate for tomato infection assay are presented in Supplementary Fig.5.

Fig. 6. Anti-cfa6 and anti-hrpL small RNA species, but not corresponding unprocessed CFA6/HRPL dsRNA precursors, are the RNA entities responsible for AGS and for the suppression of stomatal reopening

a. Accumulation level of IR-CFA6/HRPL transcripts in Col-0, dcl2-1 dcl3-1 dcl4-2 (dcl234), IR-CFA6/HRPL\#4 (\#4) and IR-CFA6/HRPL\#4 in dcl234 mutant background (\#4 $\mathrm{x}$ dcl234) was assessed by RT-qPCR. Ubiquitin was used as a control. The mean and standard deviation of three independent experiments are represented here. b. Accumulation level of anti-cfa6 and anti-hrpL siRNAs in the same genotypes presented in a. were assessed by low molecular weight northern blot analysis. U6 was used as a loading control. c. Total RNA extract from \#4 $\mathrm{x}$ dcl234 plants does not alter the transcript accumulation levels of cfa6 and hrpL. Pto WT cells were incubated in vitro for 8 hours with $20 \mathrm{ng} \mu \mathrm{l}^{-1}$ of total RNAs extracted from the same genotypes described in a. Accumulation levels of $c f a 6$ and $h r p L$ transcripts was assessed by RT-qPCR analysis using gyrA as a control. Error bars indicate the standard deviations of values from three independent experiments. d. Accumulation level of anti-cfa6 and anti-hrpL siRNAs in the indicated genotypes along with Col-0 plants incubated with total RNAs derived 
from the same genotypes for 1 hour were assessed by low molecular weight northern blot analysis. U6 was used as a loading control. Another biological replicate is presented in Supplementary Fig.6b. e. Total RNA extract from \#4 x dcl234 plants does not suppress Pto DC3000-induced stomatal reopening response. Col-0 leaves were treated with water or $20 \mathrm{ng}$ $\mu 1^{-1}$ of total RNA extracts from the same genotypes used in a. and incubated with Pto WT for 3 hours. Stomatal aperture was measured and analyzed as described in Fig.3a. Two other biological replicates are presented in Supplementary Fig.6c. f. Small RNA species, but not the corresponding long RNA species, from IR-CFA6/HRPL\#4 plants suppress stomatal reopening to the same extent as total RNAs extracted from these plants. The experiment was conducted as in d. but with total, long ( $>200 \mathrm{nt})$ or small $(<200 \mathrm{nt})$ RNA fractions, which were separated from total RNAs of IR-CFA6/HRPL\#4 plants. Two other biological replicates are presented in Supplementary Fig.7c. g. Agarose gel picture of ethidium bromide stained in vitro synthesized (IVS) long and small RNA duplexes. h. In vitro-synthesized (IVS) sRNAs, but not the corresponding long dsRNAs derived from CFA6/HRPL chimeric construct, suppressed stomatal reopening. The experiment was conducted as in d. but with in vitro synthesized- (IVS), -long or -small RNA species. Long and small RNAs derived from CYP51 were used as negative controls. Two other biological replicates are presented in Supplementary Fig.7d. i. In vitro synthesized (IVS) antibacterial siRNAs significantly alter the transcript accumulation levels of $c f a 6$ and $h r p L$. Accumulation levels of $c f a 6$ and $h r p L$ transcripts were assessed by RT-qPCR analysis using gyrA as a control. Error bars indicate the standard deviations of values from three independent experiments. For all the experiments, statistically significant differences were assessed using ANOVA test (ns: pvalue $>0.05 ; *$ : p-value $<0.05, * *$ : p-value $<0.01, * * * *$ : p-value $<0.0001)$. 
bioRxiv preprint first posted online Dec. 3, 2019; doi: http://dx.doi.org/10.1101/863902. The copyright holder for this preprint

Fig. 7. Anti-hrpL siRNAs are causal for the silencing of Pto DC3000 hrpL gene and for the suppression of stomatal reopening

a. Sequence of WT $h r p L$ (99-348 nt) selected to generate the inverted repeat transgene (highlighted in yellow) was aligned with the sequence of mutated $h r p L$ (mut $h r p L$ ) designed to contain as many silent mutations as possible in the siRNA targeted region (highlighted in grey). b. Unique reads mapping to $h r p L$ gene of Pto DC3000 in the two sRNA libraries presented in Fig.2d were used for BLAST analysis. The 500 most abundant anti-hrpL siRNA reads produced by IR-CFA6/HRPL\#4 plants were selected and blasted against the WT and mut $h r p L$ sequences depicted in a. The free energy of binding for each read paired with its top target (lowest $e$-value) was calculated using RNAup and is presented here. Few siRNA-target pairs were above the blast threshold (e-value $<10)$ when the anti-hrpL siRNA reads were blasted against the mut $h r p L$ sequence (right panel). Thermodynamic energy analysis of these few siRNA-target interactions revealed a mean free energy of binding that was roughly two times higher than the one obtained for the 500 siRNA-target pairs retrieved with the WT hrpL sequence (left panel), supporting an impaired binding of the anti-hrpL siRNAs towards the mut $h r p L$ sequence. c. Schematic representation of the Pto $\Delta h r p L$ strain along with the complementation strains generated upon transformation with the plasmids encoding WT $h r p L$ or mut $h r p L$, respectively under the control of the constitutive promoter NPTII. d. In vitro AGS assay showing that the Pto $4 h r p L$ WT $h r p L$ strain is sensitive to antibacterial RNAs while the Pto $\Delta h r p L$ mut $h r p L$ is refractory to these RNA entities. Pto $\Delta h r p L$ WT $h r p L$ (left panel) and Pto $\Delta$ hrpL mut hrpL (right panel) bacterial strains were incubated with total RNAs extracted from CV or IR-CFA6/HRPL \#4 plants for 8 hours. Accumulation of WT hrpL and mut $h r p L$ transcripts was analyzed by RT-qPCR. The mRNA levels were quantified relative to the level of gyrA transcripts. Error bars indicate the standard deviations of values from three independent experiments. e. Accumulation of anti-cfa6 and anti-hrpL siRNAs was 
bioRxiv preprint first posted online Dec. 3, 2019; doi: http://dx.doi.org/10.1101/863902. The copyright holder for this preprint

assessed by low molecular weight northern analysis using total RNA extracts from $N$. benthamiana plants transiently expressing $35 \mathrm{~S}_{p r o}: \mathrm{IR}-H R P L, 35 \mathrm{~S}_{p r o}: \mathrm{IR}-C F A 6 / H R P L$ and from non-transformed N. benthamiana leaves. U6 was used as a loading control. f. Both the WT $h r p L$ and mut $h r p L$ constructs fully complemented Pto $\Delta h r p L$ for its ability to reopen stomata and only the PtoAhrpL mut hrpL strain is refractory to anti-hrpL siRNA action. Col-0 leaves were treated with total RNAs extracted either from $N$. benthamiana alone or from $N$. benthamiana expressing the inverted repeat IR-HRPL and stomatal reopening response was

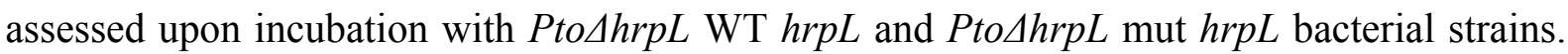
Stomatal reopening response was assessed as described previously. Two other biological replicates are presented in Supplementary Fig.9a. 9b g. Accumulation of anti-hrpL siRNAs was assessed by low molecular weight northern analysis using total RNA extracts from Arabidopsis stable transgenic lines expressing the $35 \mathrm{~S}_{p r o}$ :IR-HRPL transgene. U6 was used as a loading control. h. Col-0 and IR-HRPL\#1 and \#4 leaves were treated with water as Mock or indicated bacterial strains for 3 hours prior to stomata aperture measurements. Stomatal reopening response was assessed as described previously. Another biological replicate is presented in Supplementary Fig.9c. For all the stomata experiments, $\mathrm{n}=$ number of stomata analyzed per condition. Statistical significance for all the experiments was assessed using the ANOVA test (ns: p-value $>0.05 ; * *$ : p-value $<0.001 ; * * * *$ : p-value $<0.0001$ ).

Fig. 8. Apoplastic fluid and apoplastic extracellular vesicles (EVs) from Arabidopsis IRCFA6/HRPL plants trigger suppression of Pto DC3000-induced stomatal reopening

a. Apoplastic EVs from the Arabidopsis transgenic lines IR-CYP51 (CV) and IR-CFA6/HRPL \#4 (\#4) were isolated by collecting the apoplastic fluids (APF) from these plants and subjecting them to ultracentrifugation at $40,000 \mathrm{~g}$ as previously described ${ }^{44}$. The population of particles from the recovered P40 fractions were analyzed using Nanoparticle Tracking 
bioRxiv preprint first posted online Dec. 3, 2019; doi: http://dx.doi.org/10.1101/863902. The copyright holder for this preprint

(which was not peer-reviewed) is the author/funder, who has granted bioRxiv a license to display the preprint in perpetuity.

All rights reserved. No reuse allowed without permission.

Analysis (NTA). The number of particles, the size of particles according to their diameter (in $\mathrm{nm}$ ), and the median size of the particles are depicted for each sample. b. Both the APF and the P40 fractions triggered full suppression of stomatal reopening. Stomatal aperture was measured in Col-0 leaves that were incubated for 1 hour with the above APF or P40 fractions before being inoculated with Pto WT for 3 hours. Results from another biological replicate are presented in Supplementary Fig.10e, 10f. For the stomata experiment, $\mathrm{n}=$ number of stomata analyzed per condition and statistical significance was assessed using the ANOVA test (ns: p-value $>0.05 ; * * * *$ : p-value $<0.0001$ ). 
bioRxiv preprint first posted online Dec. 3, 2019; doi: http://dx.doi.org/10.1101/863902. The copyright holder for this preprintstogi et al., Figure 2
(which was not peer-reviewed) is the author/funder, who has granted bioRxiv a license to display the preprint in (which was not peer-reviewed) is the author/funder, who has granted bioRxiv a license to
All rights reserved. No reuse allowed without permission.

a

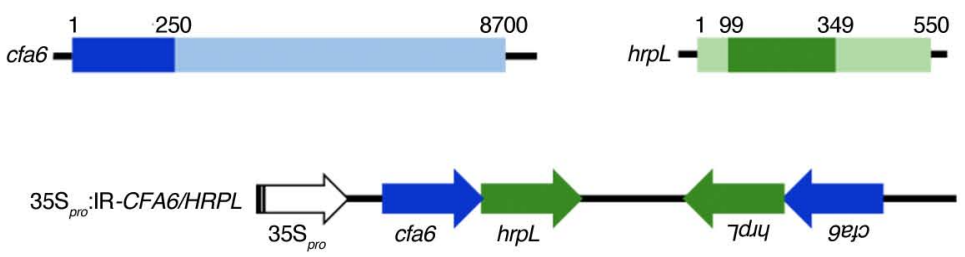

b

$35 S_{p r o}: I R-C F A 6 / H R P L$
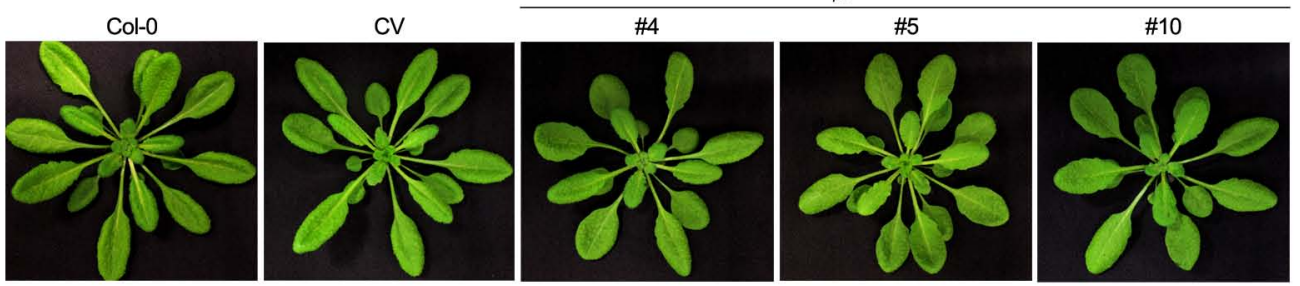

C

d

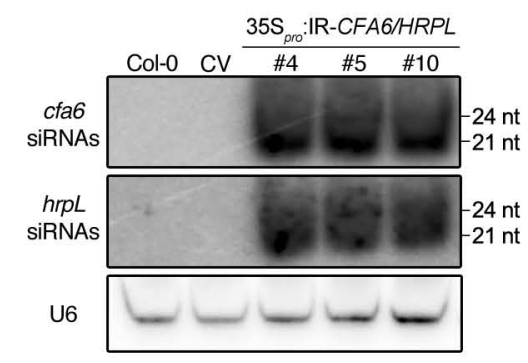

e

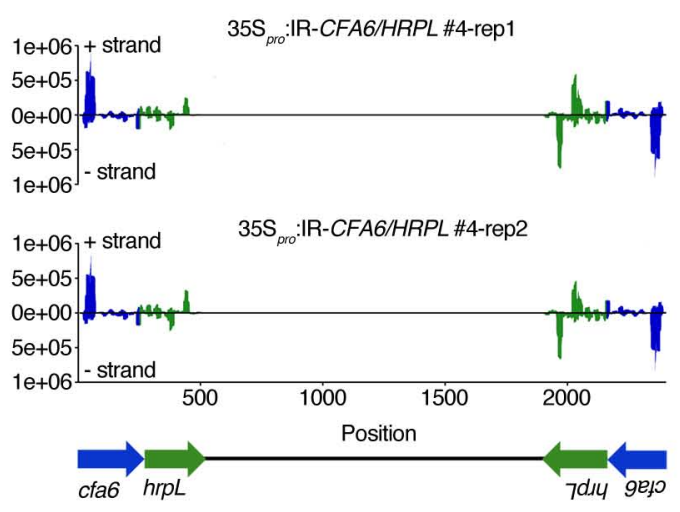

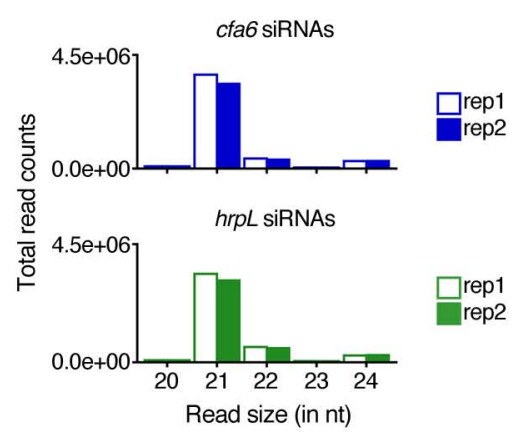

f

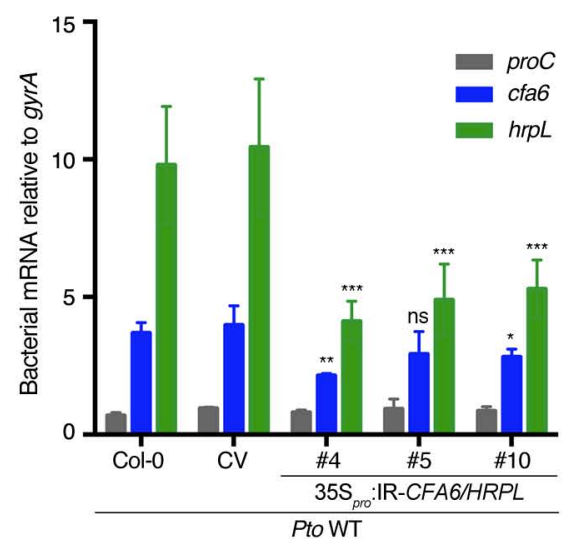

Fig. 2. Stable expression of the IR-CFA6/HRPL inverted repeat transgene in Arabidopsis triggers silencing of Pto DC3000 cfa6 and $h r p L$ genes during infection a. Schematic representation of the Pto DC3000 genes cfa6 (in blue) and $h r p L$ (in green). The 250 bp regions of cfa6 (1-250 nt) and $h r p L$ (99-348 nt) were used to generate the chimeric hairpin construct under the control of the constitutive $35 \mathrm{~S}$ promoter. $\mathbf{b}$. Representative pictures of five-week old Arabidopsis Col-0 plants and of independent homozygous transgenic plants expressing the $35 \mathrm{~S}_{\text {pro }}: \mathrm{IR}-\mathrm{CYP} 51$ (Control vector: $\mathrm{CV}$ ) or the $35 \mathrm{~S}_{\text {pro: }}$ :IR-CFA6/HRPL transgenes. c. Accumulation level of anti-cfa6 and anti-hrpL siRNAs for the Arabidopsis plants depicted in b. were detected by low molecular weight northern blot analysis. U6 was used as a loading control. d. Size distribution and abundance of 20-24 nt long sRNA reads form IR-CFA6/HRPL\#4 transgenic line that mapped to the cfa6 (upper panel) and hrpL (lower panel) regions of the IR-CFA6/HRPL sequence. Data from two biological replicates (rep1 and rep2) are presented. e. The coverage of sRNA reads which is computed as total count of mapped reads across the IRCFA6/HRPL inverted repeat is depicted and include both the plus and minus strand of the construct. f. Pto DC3000 hrpL mRNA accumulation is significantly decreased in the three independent IR-CFA6/HRPL-infected transgenic plants compared to Col-0- and CV-infected plants. Arabidopsis plants depicted in b. were dip-inoculated with $10^{8}$ cfu $\mathrm{ml}^{-1}$ Pto DC3000 WT strain and at 3 days post-infection (dpi), bacterial transcript levels of proC, cfa6 and $h r p L$ were monitored by quantitative RT-PCR analysis. These mRNA levels are quantified relative to the level of bacterial gyrA transcripts. Error bars indicate the standard deviations of mRNA values obtained in three independent experiments. Statistically significant differences were assessed using ANOVA test (ns: $p$-value $>0.05 ;{ }^{*}: p$-value $<0.05,{ }^{* *}$ : $p$-value $<0.01,{ }^{* \star *}: p$-value $<0.001$ ). 
bioRxiv preprint first posted online Dec. 3, 2019; doi: http://dx.doi.org/10.1101/863902. The copyright holder forshisprespintastogi et al., Figure 4
(which was not peer-reviewed) is the author/funder, who has granted bioRxiv a license to display the preprint iRpequity. (which was not peer-reviewed) is the author/funder, who has granted bioRxiv a license to
All rights reserved. No reuse allowed without permission.

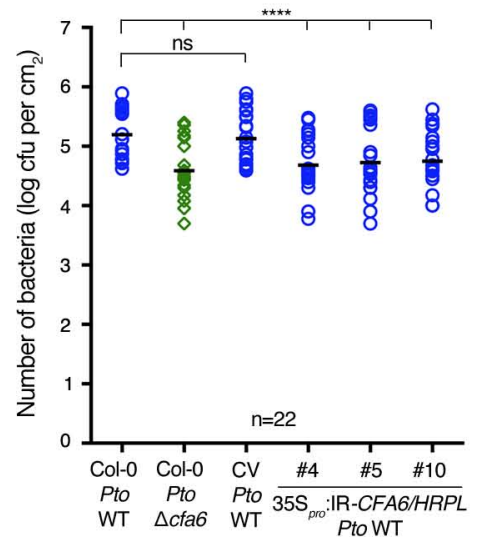

C

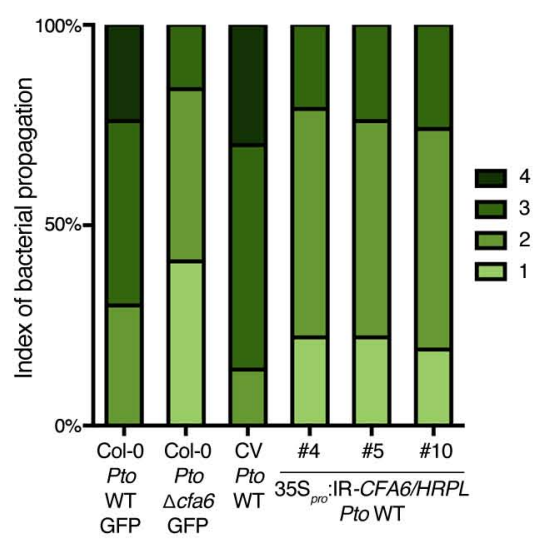

b

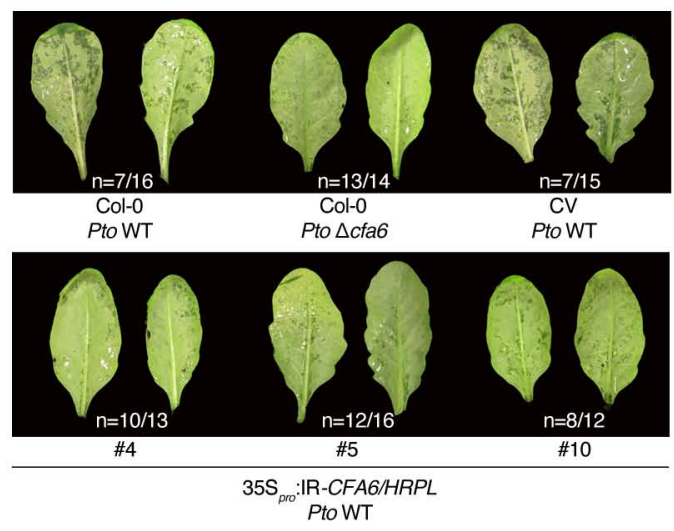

d

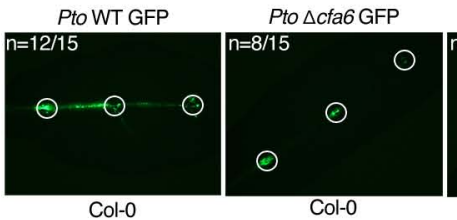

Pto WT GFP

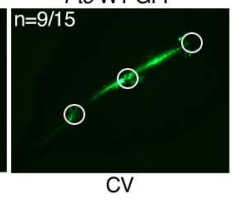

Pto WT GFP

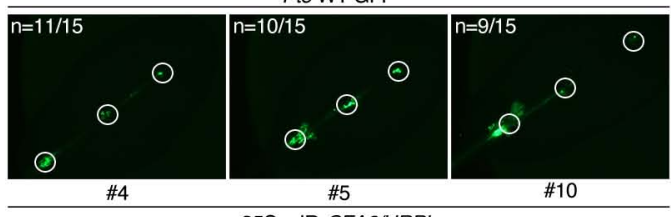

$35 S_{p p}: I R-C F A 6 / H R P L$

Fig. 4. Stable expression of the IR-CFA6/HRPL inverted repeat transgene in Arabidopsis inhibits the ability of Pto DC3000 to multiply in the apoplast, to mount water-soaking symptoms and to spread in the leaf vasculature

a. Arabidopsis IR-CFA6/HRPL transgenic lines exhibit a significantly reduced Pto WT titer when compared to Col-0 and CV-infected plants. Col-0, CV and IR-CFA6/HRPL\#4, $\# 5$ and \#10 plants were dip-inoculated with Pto WT-GFP and Col-0 plants were dip-inoculated with the Pto $\Delta$ cfa6-GFP strain. Bacterial titers were monitored at 2 days post-infection (dpi). Four leaves from three plants per condition and from three independent experiments $(n)$ were considered for the comparative analysis. Statistical significance was assessed using the ANOVA test (ns: p-value>0.05; ****: p-value<0.0001). b. IR-CFA6/HRPL\#4, \#5 and \#10 transgenic plants exhibit reduced water-soaking symptoms in comparison to Col-0 and CV plants. Representative leaf pictures of water-soaking symptoms were taken 1 day after dip-inoculation with Pto WT-GFP or Pto $\triangle$ cfa6-GFP strain. c. \& d. IR-CFA6/HRPL\#4, \#5 and \#10-infected plants exhibit reduced vascular spreading of Pto WT compared to Col-0- and CV-infected plants. Plants were woundinoculated in midveins with Pto WT-GFP and Col-0 was wound-inoculated with Pto $\triangle$ cfa6-GFP. White circles indicate the site of wound-inoculation in the midvein. To index the spreading of bacteria from the inoculation sites, GFP fluorescence signal was observed under UV light and pictures were taken at 3 dpi. When the bacteria propagated away from any of the three inoculation sites, it was indexed as propagation with 4 corresponding to the highest propagation index. Pictures from three biological replicates were taken into consideration and one representative picture is depicted. 


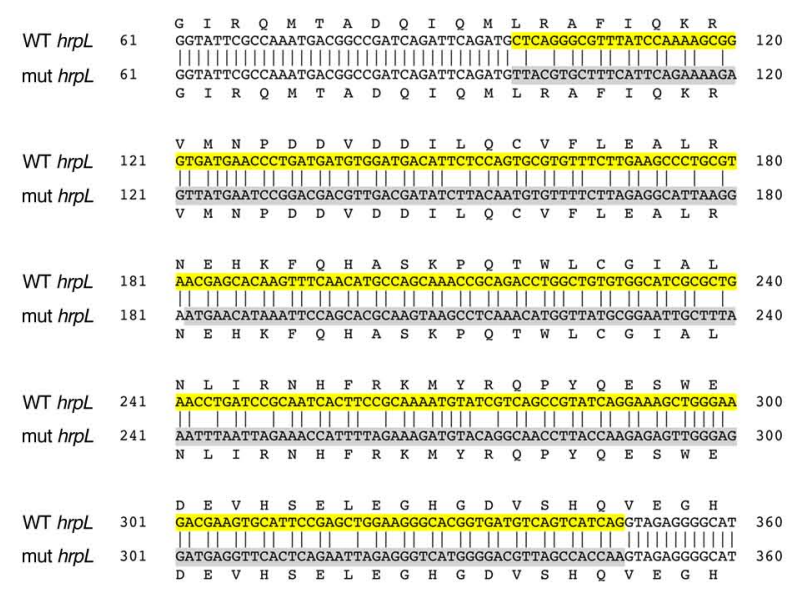

d
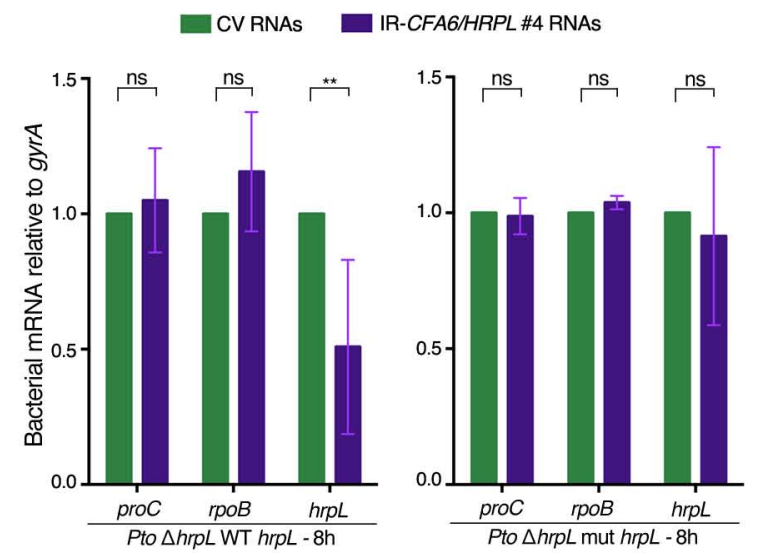

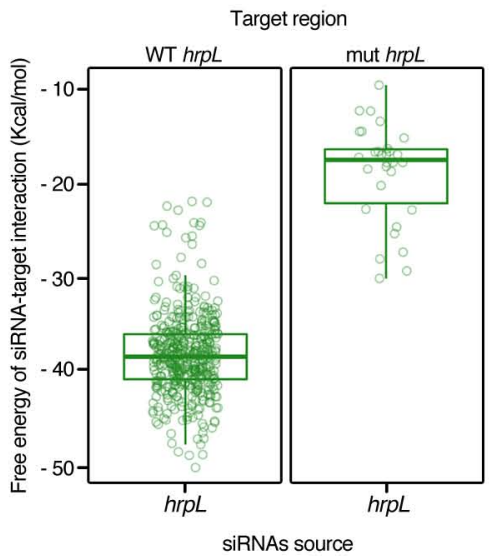

f g

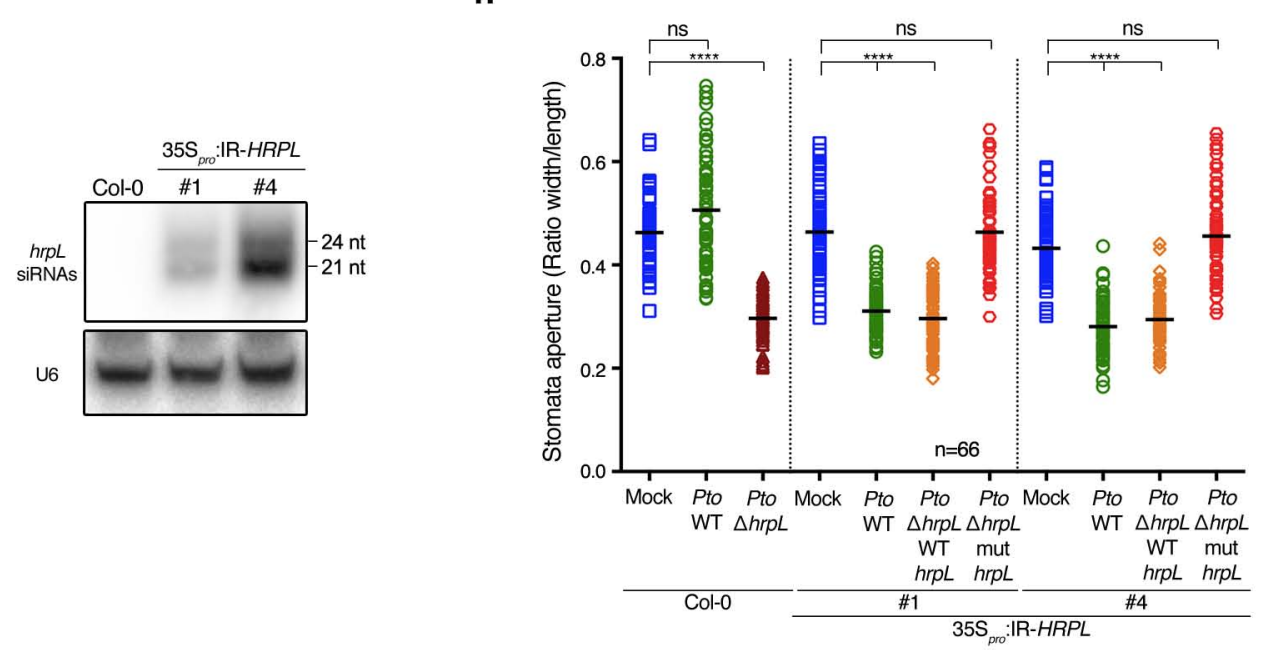

h
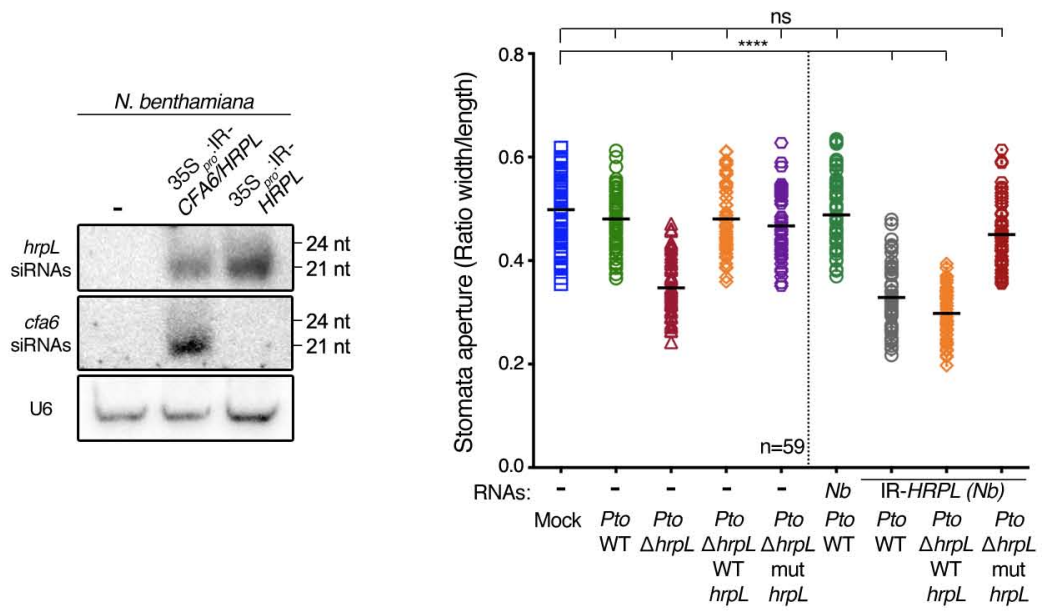

Fig. 7. Anti-hrpL siRNAs are causal for the silencing of Pto $D C 3000$ hrpL gene and for the suppression of stomatal reopening

a. Sequence of WT hrpL (99-348 nt) selected to generate the inverted repeat transgene (highlighted in yellow) was aligned with the sequence of mutated $h r p L$ (mut $h r p L$ ) designed to contain as many silent mutations as possible in the siRNA targeted region (highlighted in grey). b. Unique reads mapping to $h r p L$ gene of $P$ to DC3000 in the two sRNA libraries presented in Fig. 2c were used for BLAST analyses. The 500 most abundant anti- $h r p L$ siRNA reads produced by IR-CFA6/HRPL\#4 plants were selected and blasted against the WT hrpL and mut $h r p L$ sequences depicted in a. The free energy of the top targets with lowest e-values for each read/target pair was calculated using RNAup and is presented here. Few siRNA-target pairs were above the blast threshold when the anti-hrpL siRNA reads were blasted against the mut $h r p L$ sequence (right panel). Thermodynamic energy analysis of these few siRNA-target interactions revealed a mean free energy that was roughly two times higher than the one obtained for the 500 siRNA-target pairs retrieved with the WT hrpL sequence (left panel), supporting an impaired binding of the anti-hrpL siRNAs towards the mut $h r p L$ sequence. c. Schematic representation of the Pto $\Delta$ hrpL strain along with the complementation strains generated upon transformation with the plasmids encoding WT $h r p L$ or mut $h r p L$, respectively under the control of the constitutive promoter NPTII. d. In vitro AGS assay showing that the Pto $\triangle h r p L$ WT hrpL strain is sensitive to antibacterial RNAs while the Pto $\triangle h r p L$ mut $h r p L$ is refractory to these RNA entities. Pto $\triangle h r p L$ WT $h r p L$ (left panel) and Pto $\triangle h r p L$ mut $h r p L$ (right panel) bacterial strains were incubated with total RNAs extracted from CV or IR-CFA6/HRPL\#4 plants for 8 hours. Accumulation of WT $h r p L$ and mut $h r p L$ transcripts was analyzed by RT-qPCR. The mRNA levels were quantified relative to the level of gyrA transcripts. Error bars indicate the standard deviations of values from three independent experiments. e. Accumulation of anti-cfa6 and anti- $h r p L$ siRNAs was assessed by low molecular weight northern analysis using total RNA extracts from $N$. benthamiana plants transiently expressing $35 \mathrm{~S}_{\text {pro }}: \mathrm{IR}-H R P L, 35 \mathrm{~S}_{\text {pro }}: \mathrm{IR}-\mathrm{CF} A 6 / \mathrm{HRPL}$ and from non-transformed $N$. benthamiana leaves $(\mathrm{Nb})$. U6 was used as a loading control. f. Both the WT $h r p L$ and mut $h r p L$ constructs fully complemented Pto $\Delta h r p L$ for its ability to reopen stomata and only the Pto $\Delta h r p L$ mut $h r p L$ strain is refractory to anti-hrpL siRNA action. Col-0 leaves were treated with total RNAs extracted either from $N$. benthamiana alone or from $N$. benthamiana expressing the inverted repeat IR-HRPL and stomatal reopening response was assessed upon incubating with Pto $\triangle h r p L$ WT $h r p L$ and $P t o \Delta h r p L$ mut $h r p L$ bacterial strains. Stomatal reopening response was assessed as described previously. Two other biological replicates are presented in Supplementary Fig. 9a. g. Accumulation of anti-hrpL siRNAs was assessed by low molecular weight northern analysis using total RNA extracts from Arabidopsis stable transgenic lines expressing the $35 \mathrm{~S}_{\text {pro }}$ :IR-HRPL transgene. U6 was used as a loading control. h. Col-0 and IR-HRPL, \#1 and \#4 leaves were treated with water as Mock or indicated bacterial strains for 3 hours prior to stomata aperture measurements. Stomatal reopening response was assessed as described previously. Another biological replicate is presented in Supplementary Fig. $9 \mathrm{~b}$. For all the stomata experiments, $\mathrm{n}=$ number of stomata analysed per condition. Statistical significance for all the experiments was assessed using the ANOVA test (ns: $p$-value $>0.05 ;{ }^{* \star}: p$-value $<0.001 ;{ }^{* \star \star *}$ : p-value $<0.0001$ ) 


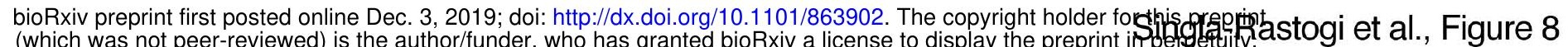
(which was not peer-reviewed) is the author/funder, who has granted bioRxiv a license to
All rights reserved. No reuse allowed without permission.

P40 fraction

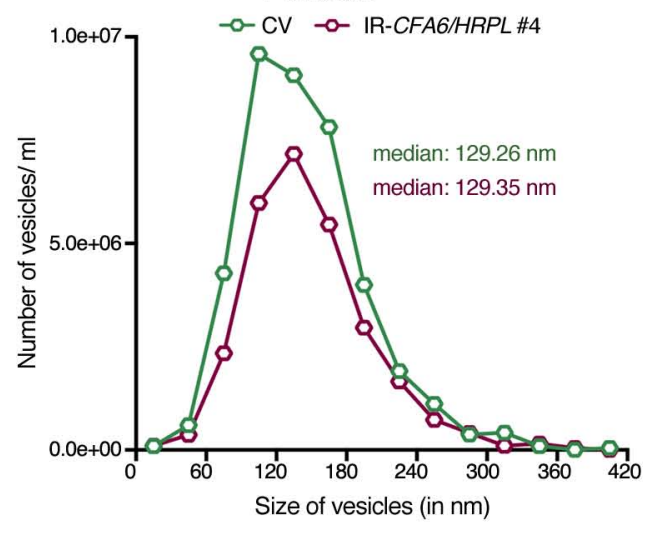

b

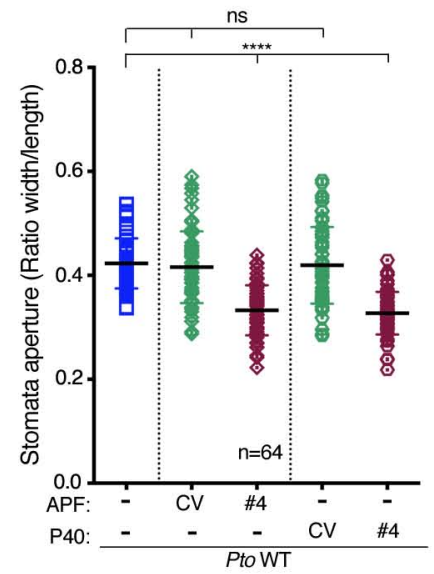

Fig 8. Apoplastic fluid and apoplastic extracellular vesicles (EVs) from Arabidopsis IR-CFA6/HRPL plants trigger suppression of Pto DC3000-induced stomatal reopening

a. Apoplastic EVs from the Arabidopsis transgenic lines IR-CYP51 (CV) and IR-CFA6/HRPL \#4 (\#4) were isolated by collecting the apoplastic fluids (APF) from these plants and subjected them to ultracentrifugation at $40,000 \mathrm{~g}$ as previously described (ref). The population of particles from the recovered P40 fractions were analysed using Nanoparticle Tracking Analysis (NTA). The number of particles, the size of particles according to their diameter (in nm), and the median size of the particles are depicted for each sample. b. Both the APF and the P40 fractions triggered full suppression of stomatal reopening. Stomatal aperture was measured in Col-0 leaves that were incubated for 1 hour with the above APF or P40 fractions before being inoculated with Pto WT for 3 hours. Results of another biological replicate is presented in Supplementary Fig $10 e$ and $10 f$. For the stomata experiment, $n=$ number of stomata analysed per condition and statistical significance was assessed using the ANOVA test (ns: $p$-value $>0.05$; ${ }^{* \star \star *}: p$ value<0.0001) 


\section{SUPPLEMENTARY INFORMATION}

Plant Small RNA Species Direct Gene Silencing in Pathogenic Bacteria as well as

Disease Protection 


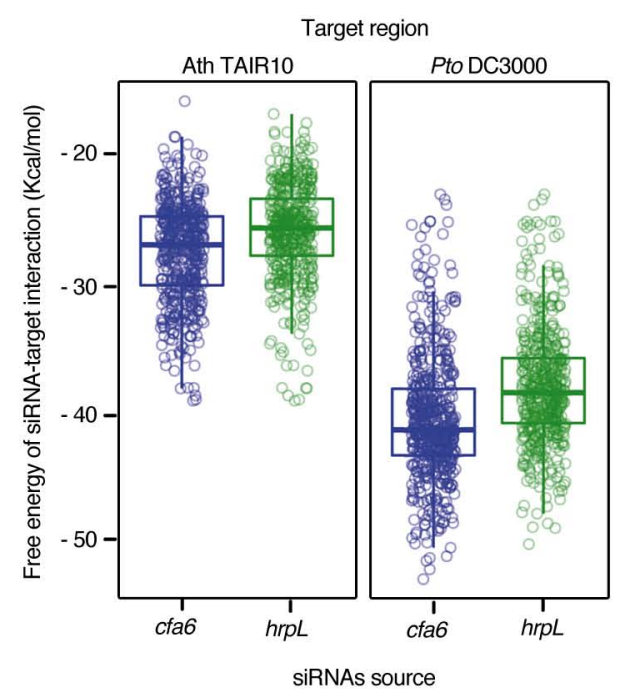

Supplementary Fig. 1. Target prediction of sequenced anti-cfa6 and anti-hrpL siRNAs in Arabidopsis and Pto DC3000

Unique reads mapping to cfa6 and $h r p L$ genes of Pto DC3000 from the two sRNA libraries presented in Fig. 2d were used for BLAST analyses. The 500 most abundant anticfa6 and anti-hrpL siRNA reads were selected and blasted against annotated Arabidopsis genes (TAIR10) and Pto DC3000 coding sequences (CDS). The free energy of the top targets with lowest e-values for each read/target pair (500 pairs) was calculated using RNAup and is presented here. Thermodynamic energy analysis revealed that the free energy of siRNA-Pto DC3000 target interactions (Kcal/mol), which are almost exclusively composed of siRNA/cfa6 and siRNA/hrpL pairs (Table S2), is significantly lower than the one from siRNA-Arabidopsis transcript interactions (Table S1), indicating that off-targets in Arabidopsis seems to be unlikely (Table S2 depicting the high e-values for all the interactions between the anti-cfa6 and anti-hrpL siRNAs and Arabidopsis genes). 


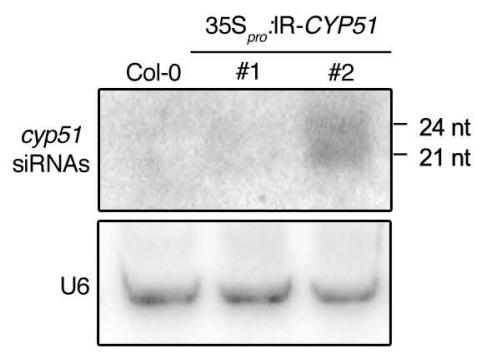

Supplementary Fig. 2. Accumulation level of anti-cyp51 siRNAs in Arabidopsis IR-CYP51 transgenic lines.

Accumulation level of anti-cyp51A/B/C siRNAs in Col-0 and in the transgenic T3 homozygous 35S pro:IR-CYP51\#1 and \#2 plants was assessed by low molecular weight northern blot analysis. U6 was used as a loading control. IR-CYP51\#2 was used as a control transgenic line (CV) for the assays presented in this study. 
a

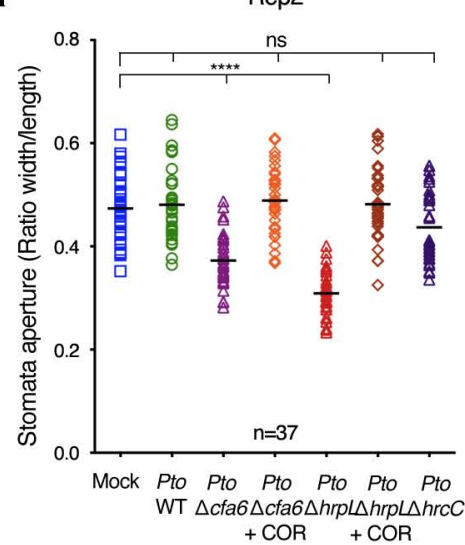

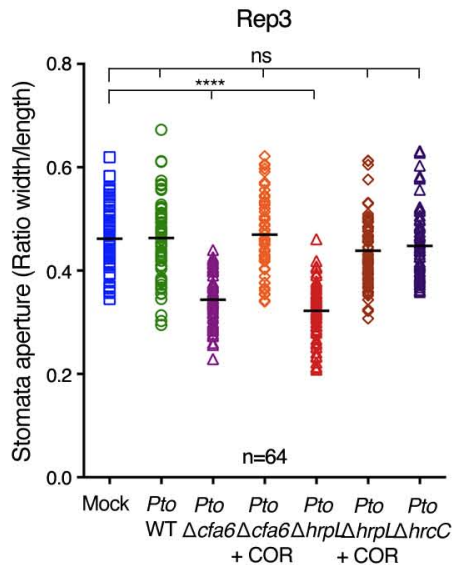

c

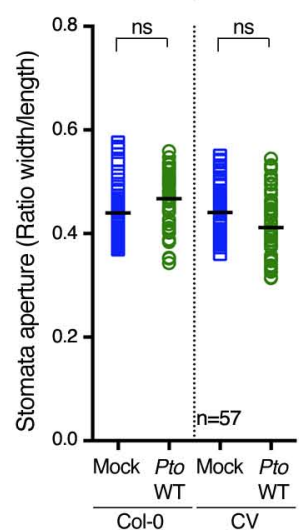

Rep3

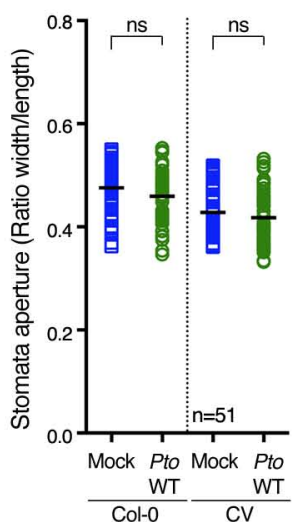

b
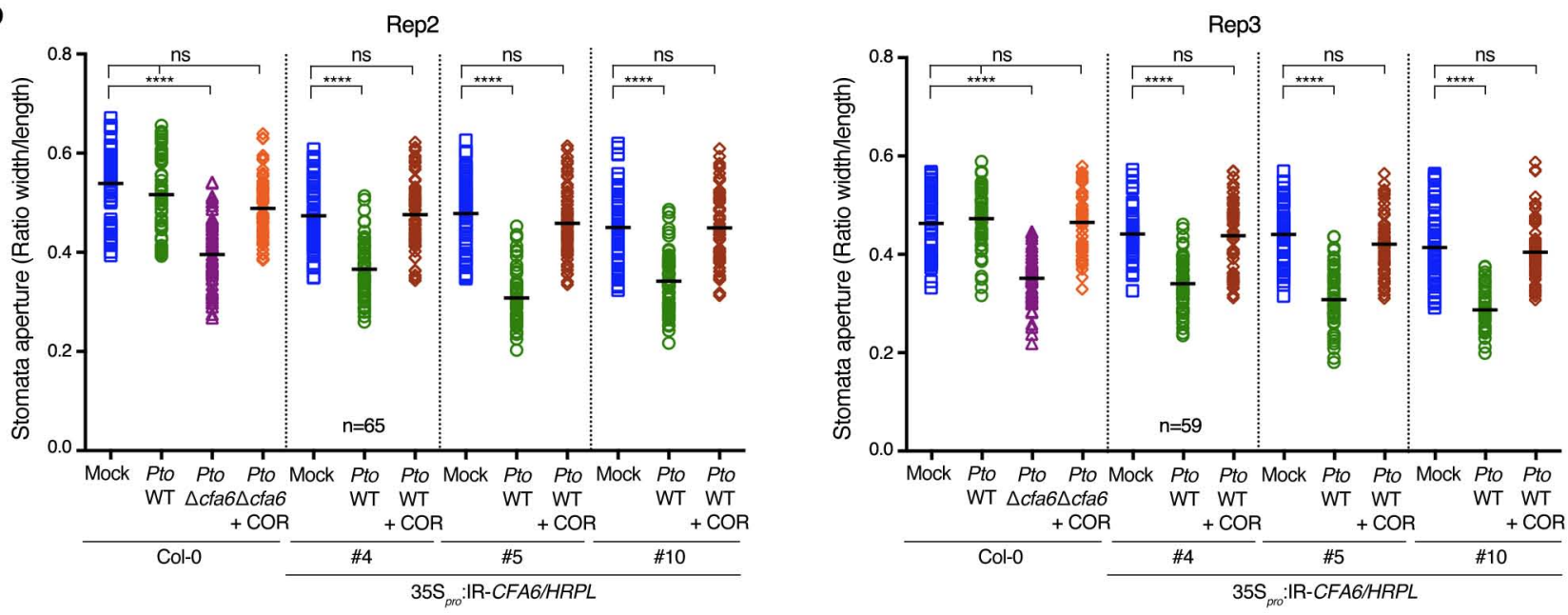

Supplementary Fig. 3. Stable expression of the IR-CFA6/HRPL inverted repeat transgene in Arabidopsis suppresses Pto DC3000-induced stomatal reopening a. Other two biological replicates of Fig. 3a: The Pto $\Delta c f a 6$ and Pto $\Delta h r p L$ strains, but not the Pto $\Delta h r c C$ strain, were impaired in their ability to reopen stomata and these phenotypes were rescued upon addition of COR. Sections of unpeeled leaves of Col-0 plants were incubated with mock solution (water) or $10^{8}$ cfu ml- ${ }^{-1}$ of Pto DC3000 WT, $\triangle c f a 6$, $\Delta h r p L$ or $\triangle h r c C$ strains for 3 hours. Stomatal aperture was assessed by computing the ratio of the width and length using Image J software. b. Other two biological replicates of Fig. 3b: Pto DC3000 WT no longer induced stomatal reopening in Arabidopsis transgenic lines overexpressing the IR-CFA6/HRPL inverted repeat transgene. Stomatal aperture measurement was conducted in Col-0 and IR-CFA6/HRPL\#4, \#5, \#10 transgenic lines infected with Pto WT strain as described in a. c. Other two biological replicates of Fig. 3c: The Pto DC3000-induced stomatal reopening response was unaltered in CV compared to Col-0 plants. Stomatal aperture measurement was conducted in Col-0 and CV plants incubated with Pto WT strain as described in $\mathrm{a}$. For all these experiments, $\mathrm{n}=$ number of stomata analyzed per condition and statistical significance was assessed using the ANOVA test (ns: $p$-value $>0.05 ;{ }^{\star \star \star \star}$ : $p$-value $<0.0001$ ). 
a

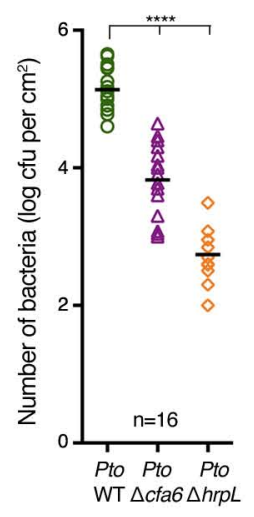

C

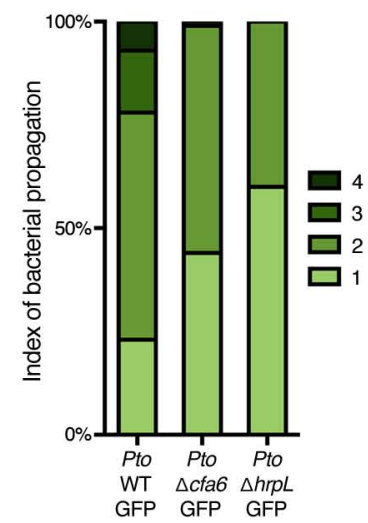

b

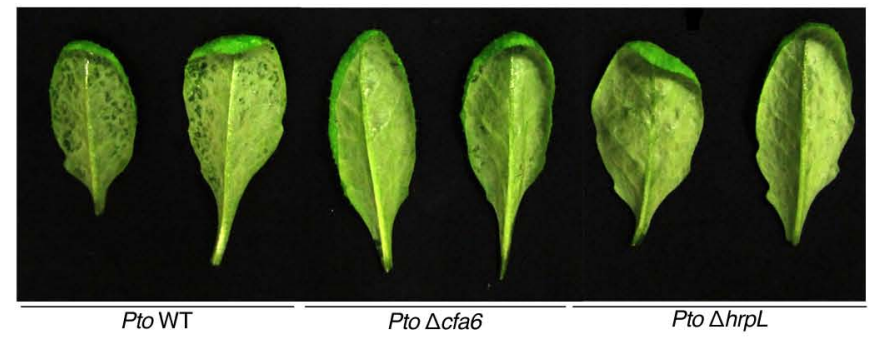

d
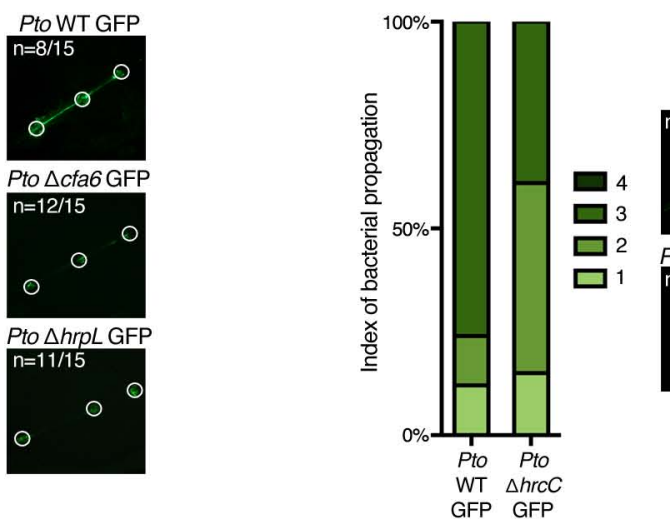

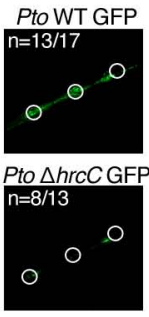

$0^{0}$

\section{Supplementary Fig. 4. Pathogenicity phenotypes of the Pto DC3000 strains deleted of either cfa6 or hrpL}

a. The Pto $\Delta$ cfa6 and Pto $\Delta$ hrpL strains are impaired in leaf apoplast multiplication in Arabidopsis. Bacterial titers in Col-0 plants dip-inoculated with $P$ to WT, Pto $\Delta c f a 6$ and $P$ to $\Delta h r p L$ strains were monitored 2 days-post inoculation (dpi). Data from two independent replicates is presented here. b. The Pto $\Delta$ cfa6 and Pto $\Delta h r p L$ strains are impaired in their ability to induce water-soaking symptoms when dip-inoculated on Arabidopsis Col-0 plants. Representative pictures of water-soaking symptoms were taken at 1 dpi. c. \& d. The Pto $\Delta$ cfa6, Pto $\Delta$ hrpL and Pto $\Delta$ hrcC mutant strains are compromised in leaf vascular propagation. Col-0 leaves were wound-inoculated in midveins with Pto WT-GFP, Pto $\Delta$ cfa6-GFP, Pto $\Delta$ hrpL-GFP or Pto $\Delta$ hrcC-GFP strains. White circles indicate the sites of wound-inoculation in the midveins. To index the spreading of bacteria from the inoculation sites, GFP fluorescence was observed under UV light and pictures were taken at $3 \mathrm{dpi}$. When the bacteria propagated away from any of the three inoculation sites, it was indexed as propagation with 4 corresponding to the highest propagation index. Pictures from three biological replicates were taken into consideration and one representative picture is depicted. $n=$ number of pictures analyzed per condition and statistical significance was assessed using the ANOVA test ( ${ }^{\star \star \star *}: \mathrm{p}$-value <0.0001). 
a

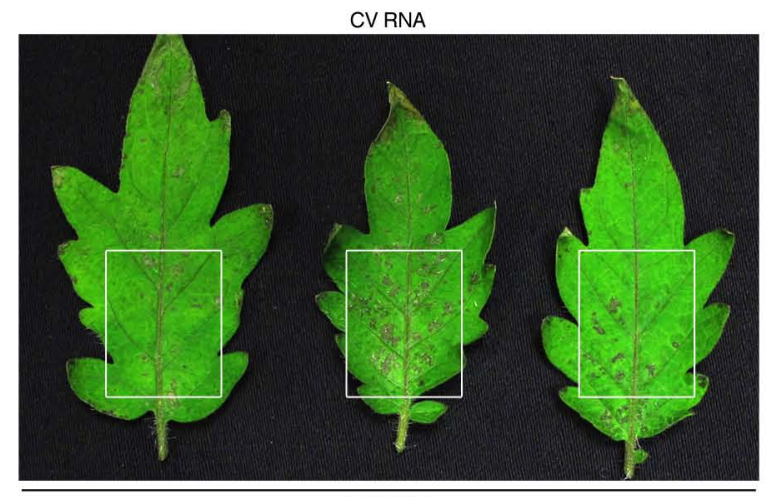

Pto WT GFP

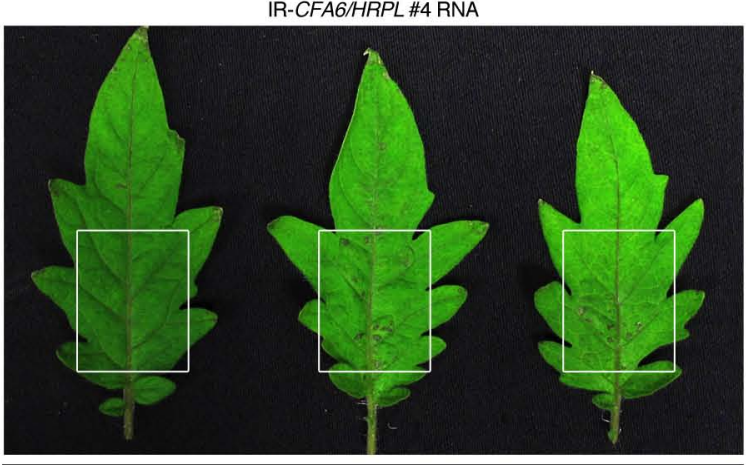

PtoWT GFP

b

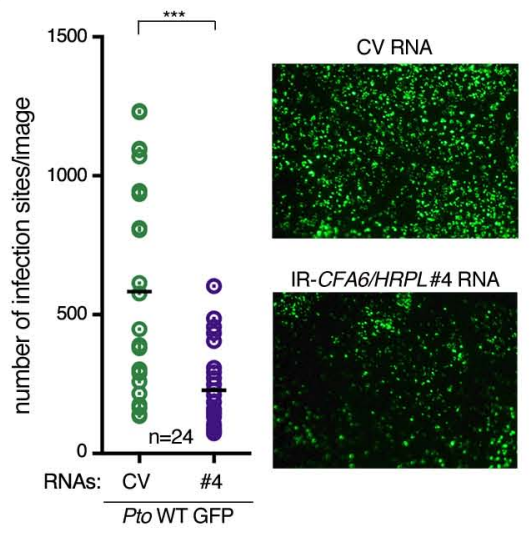

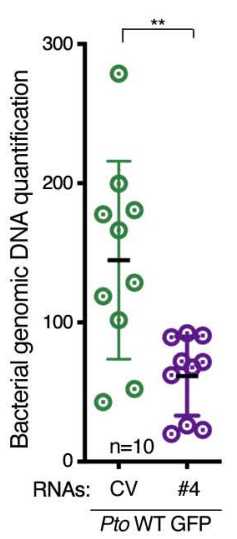

Supplementary Fig. 5. Assessing the effect of total RNAs from IR-CFA6/HRPL Arabidopsis transgenic lines applied exogenously on tomato leaves before Pto DC3000 infection

a. Additional biological replicate of Fig. 5d: The tomato leaves treated with CV total RNAs displayed more necrotic symptoms as compared to the leaves treated with IRCFA6/HRPL\#4 total RNAs. The experiment was conducted as in Fig 5c. but using five-week-old tomato (Solanum lycopersicum, cultivar 'Moneymaker') plants. Representative pictures of infected leaves in the two conditions are depicted. b. Additional biological replicate of Fig. 5e: A reduced number of Pto DC3000-GFP foci was observed in tomato leaves treated with total RNA extracts from IR-CFA6/HRPL\#4 vs CV plants. Infected-leaves were observed at 3 dpi under UV light to estimate the number of GFP loci. Left panel: Dot plot representing the number of GFP loci analyzed using Image J software from 3-4 different leaves per condition with at least 4 pictures per leaf. The values used for the analysis are from two different independent experiments. Right panel: Representative picture of the tomato leaves described in Fig $5 \mathrm{~d}$. c. Additional biological replicate of Fig. 5f: Pto WT-GFP DNA content is decreased in tomato leaves treated with total RNA extracts from IR-CFA6/HRPL\#4 vs CV plants. The level of bacterial DNA content was analyzed by qPCR using tomato Ubiquitin gene as a control. For b and c, student's t-test was performed for the comparative analysis (ns: $\mathrm{p}$-value>0.05; , ${ }^{\star *}$ : $\mathrm{p}$ value $<0.01,{ }^{* * *}: p$-value<0.001) 
Singla-Rastogi et al., Supplementary Figure 6

a

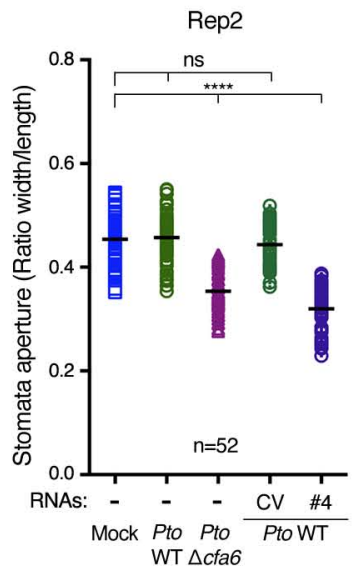

b

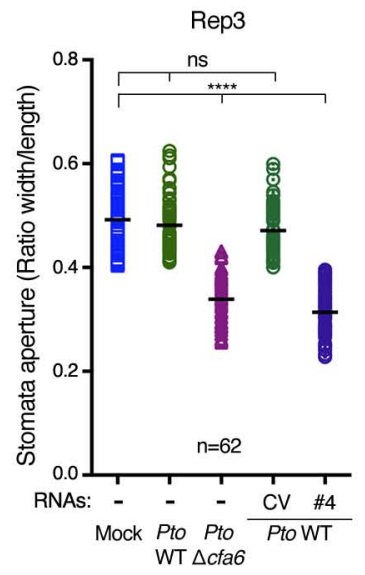

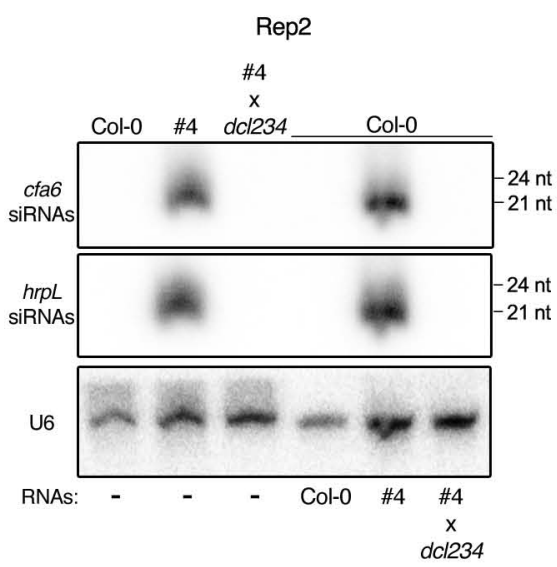

C
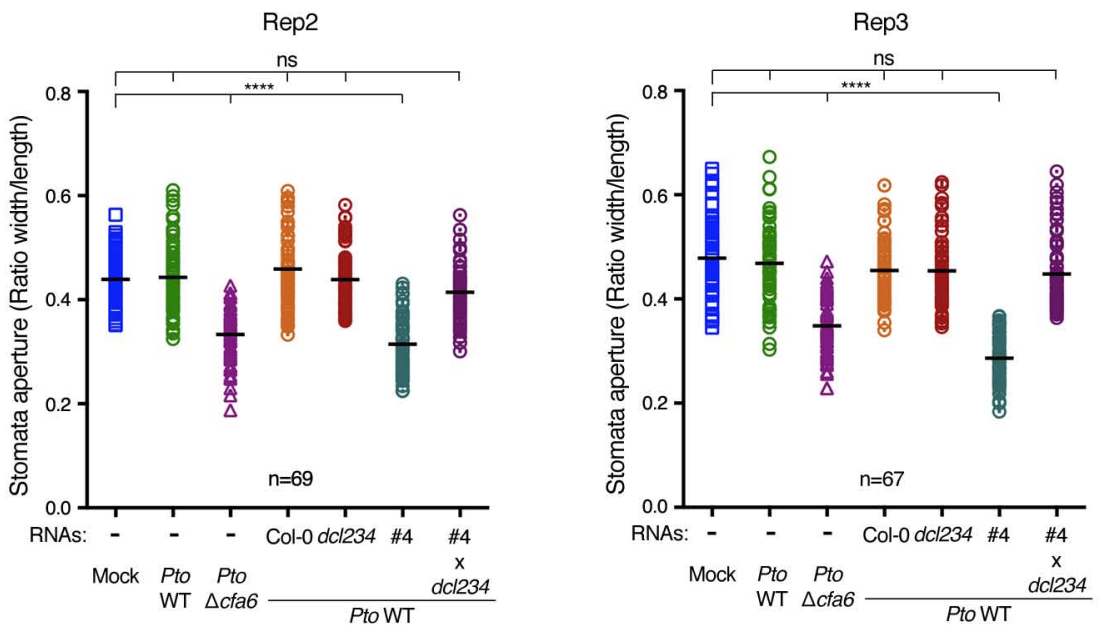

Supplementary Fig. 6. Assessing the effects of exogenously applied total RNAs from different genotypes on stomata reopening events a. Other two biological replicates of Fig. Sb: The ability of Pto WT to reopen stomata was altered upon exogenous application of total RNA extracts from IR-CFA6/HRPL plants compared to CV plants. Col-0 leaves were treated for 1 hour with water (Mock) or $20 \mathrm{ng}_{\mathrm{Hl}}^{-1}$ of total RNA extracted from CV or IR-CFA6/HRPL\#4 plants and were incubated with Pto WT for 3 hours. Stomatal aperture was measured and analyzed as described previously. b. Another biological replicates of Fig. $6 \mathrm{~d}$ : Accumulation level of anti-cfa6 and anti-hrpL siRNAs in the indicated genotypes along with Col-0 plants incubated with total RNA derived from the same genotypes for 1 hour was assessed by low molechlar weight northern blot analysis. U6 was used as a loading control. c. Other two biological replicates of Fig. Ge: Total RNA extract from \#4 x decl 234 plants does not suppress Pto DC3000-induced stomatal reopening response. Col-0 leaves were treated with water or $20 \mathrm{ng} \mathrm{ul}^{-1}$ of total RNA extracts from the same genotypes used in a. and incubated with Pto WT for 3 hours. Stomatal aperture was measured and analyzed as described previously. For all the stomata experiments, $n=$ number of stomata analyzed per conditimon and statistical significance was assessed using the ANOVA test (ns: $p$-value $>0.05 ;{ }^{* \star \star *}$ : $p$-value $<0.0001$ ). 
a

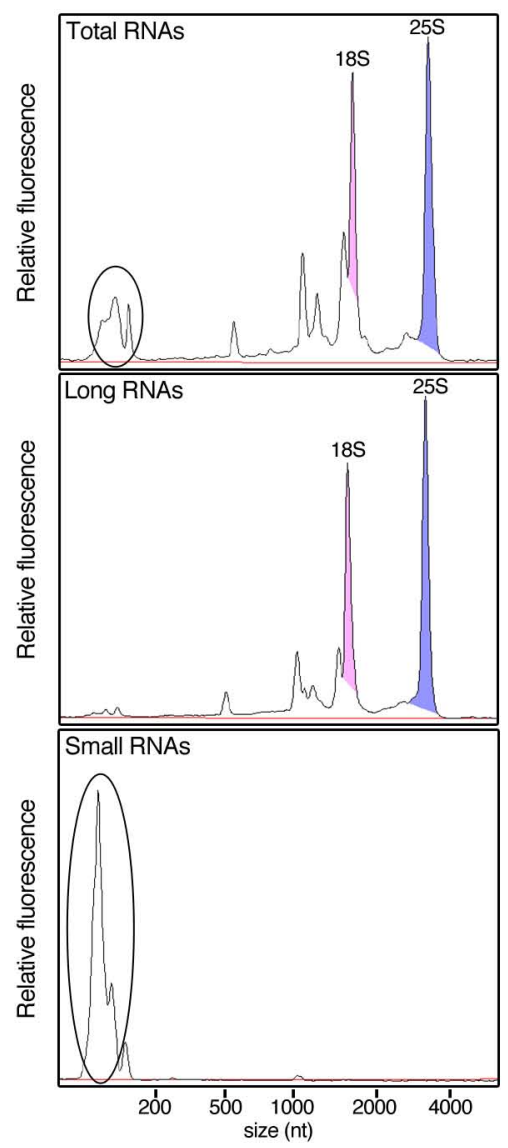

b
C

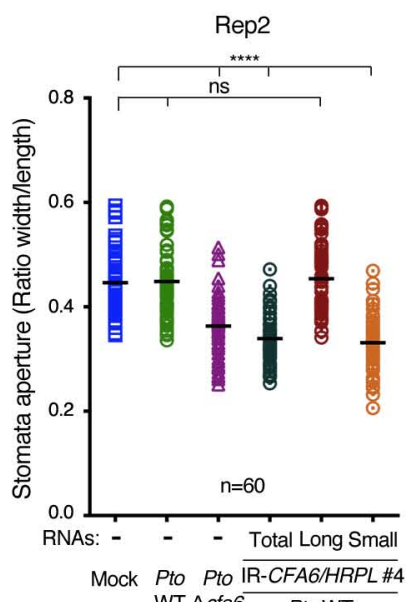

WT $\triangle$ cfa 6 Pto WT

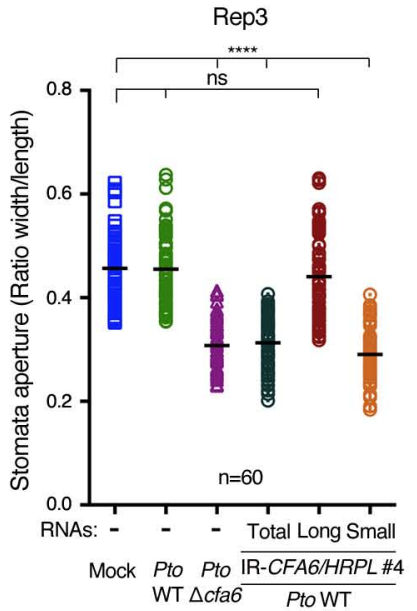

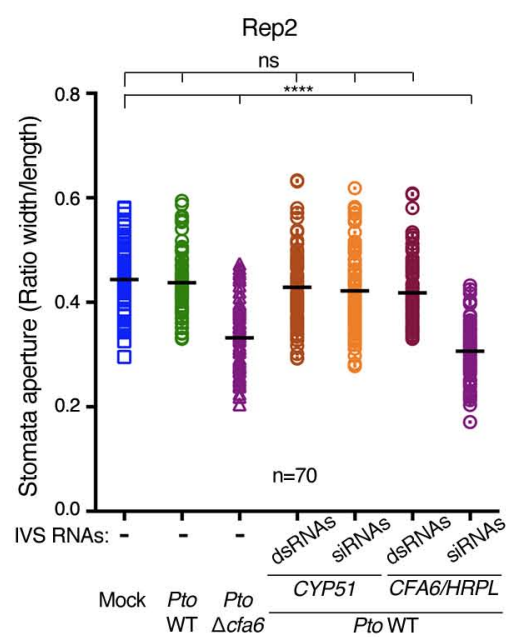

Rep3

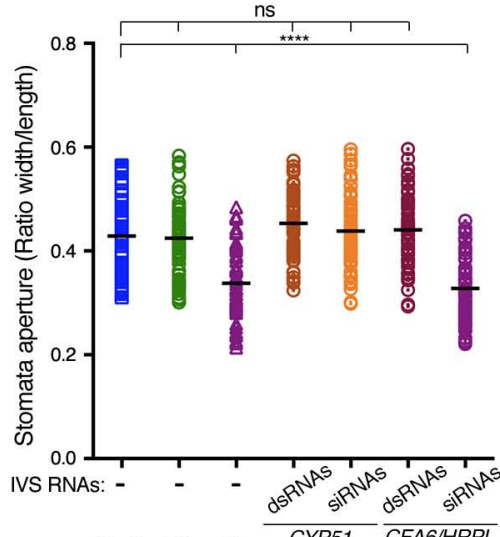

Mock Pto Pto

WT $\triangle c f a 6$
CYP51 CFA6/HRPL

PtoWT

Fig. S7. Characterization of the long and small RNA fractions separated from IR-CFA6/HRPL\#4 total RNAs or in vitro synthesized (IVS)

a. Size distribution of total, long and small RNAs from IR-CFA6/HRPL\#4 plants was determined with an agilent Bioanalyzer 2100 equipped with an RNA Nano chip. Low molecular weight RNA fractions are encircled for each sample. $18 \mathrm{~S}$ and $25 \mathrm{~S}$ ribosomal peaks are highlighted. $\mathbf{b}$. Agarose gel picture of ethidium bromide stained total, long and small RNAs used in a. c. Other two biological replicates of Fig. 6f: Small RNA species, but not the corresponding long RNA species, from IR-CFA6/HRPL\#4 plants suppress stomatal reopening to the same extent as total RNAs extracted from these plants. The experiment was conducted as in Fig $6 \mathrm{~d}$. but with total, long ( $>200 \mathrm{nt})$ or small ( $<200$ nt) RNA fractions, which were separated from total RNAs of IR-CFA6/HRPL\#4 plants. d. Other two biological replicates of Fig. $6 \mathrm{~g}$ : In vitro synthesized (IVS) sRNAs, but not the corresponding long dsRNAs derived from CFA6/HRPL chimeric construct, suppressed stomatal reopening. The experiment was conducted as in Fig. $6 \mathrm{~d}$. but with in vitro synthesized (IVS), long or small RNA species. Long and small RNAs derived from CYP51 were used as negative controls. For all the stomata experiments, $n=$ number of stomata analyzed per condition. For all the experiments, statistically significant differences were assessed using ANOVA test (ns: $p$-value $>0.05 ;{ }^{\star}: p$-value $<0.05$, ${ }^{\star *}: p-$ value $<0.01,{ }^{\star \star \star \star}: p$-value $\left.<0.0001\right)$ 

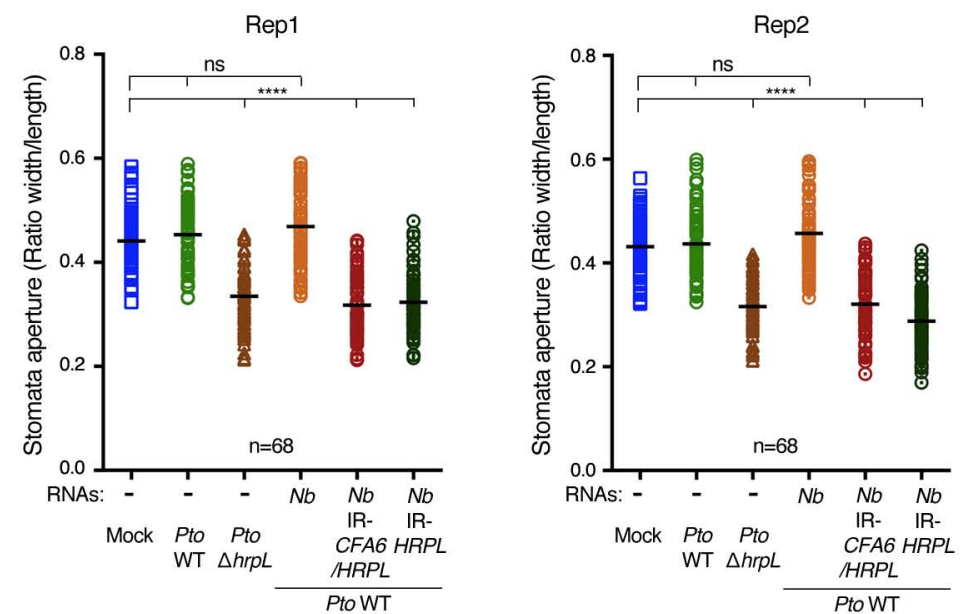

Supplementary Fig. 8. N. benthamiana total RNAs containing anti-hrpL siRNAs trigger suppression of Pto DC3000-induced stomatal reopening

Col-0 leaves were treated with total RNAs extracted either from $N$. benthamiana alone or from $N$. benthamiana expressing the inverted repeat IR-CFA6/HRPL or IR-HRPL. Stomatal reopening response was assessed by incubating Col-0 leaves with specified total RNAs before incubating with Pto DC3000 as described previously. Two biological replicates are presented here. 
a

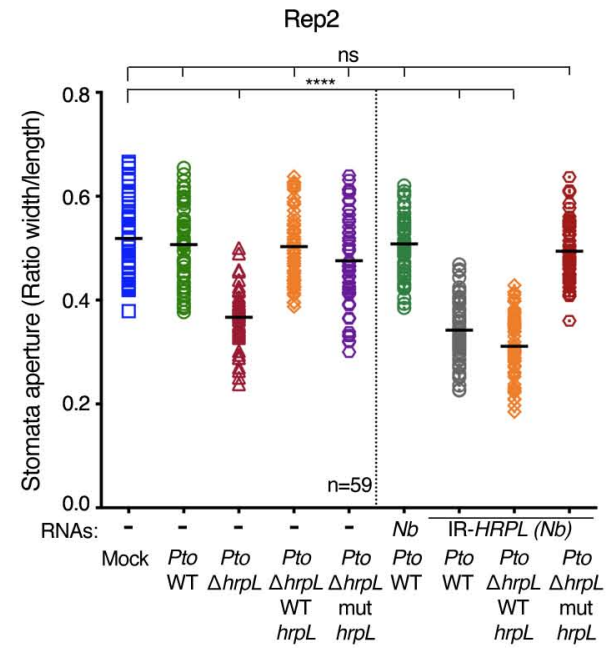

b

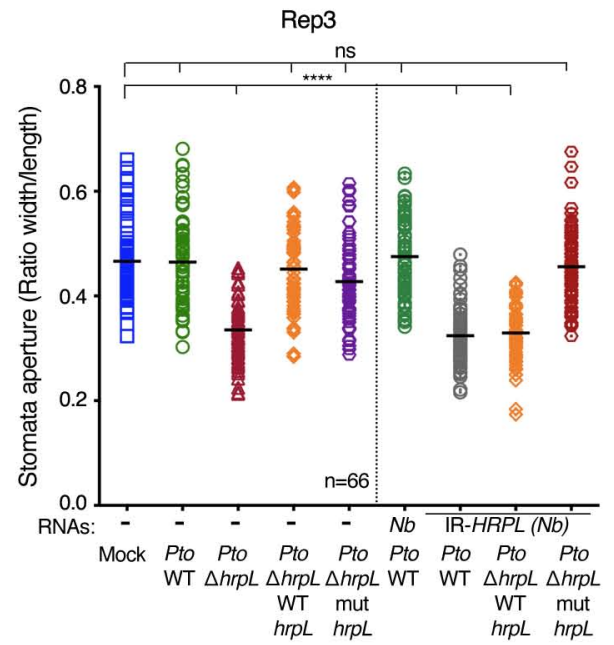

C

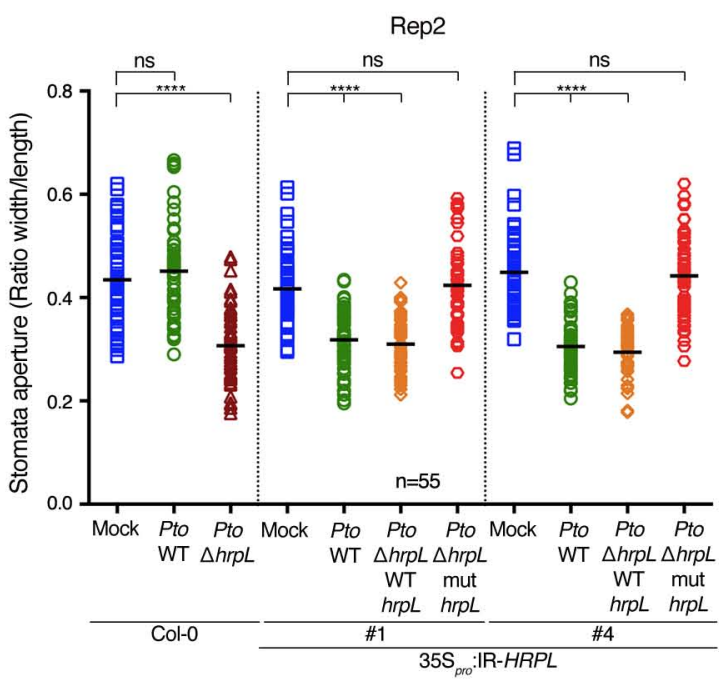

Supplementary Fig. 9. Both exogenously applied and plant-encoded anti-hrpL siRNAs are causal for the the suppression of stomatal reopening events a. \& b. Other two biological replicates of Fig. 7f. Both the WT $h r p L$ and mut $h r p L$ constructs fully complemented $P$ to $\Delta h r p L$ for its ability to reopen stomata and only the $P$ to $\Delta$ $h r p L$ mut $h r p L$ strain is refractory to anti-hrpL siRNA action. Col-0 leaves were treated or not with total RNAs extracted either from $N$. benthamiana alone or from $N$. benthamiana expressing the inverted repeat IR-HRPL and stomatal reopening response was assessed upon incubating with Pto $\Delta h r p L$ WT $h r p L$ and $P t o \Delta h r p L$ mut $h r p L$ bacterial strains for 3 hours. Stomatal reopening response was assessed as described previously. b. Other two biological replicate of Fig. 7h: Col-0 and IR-HRPL\#1 and \#4 leaves were treated with water as Mock or indicated bacterial strains for 3 hours prior to stomata aperture measurements. Stomatal reopening response was assessed as described previously. For all the experiments, statistically significant differences were assessed using ANOVA test (ns: $p$-value $>0.05 ;{ }^{* * * *}$ p-value<0.0001). 
a
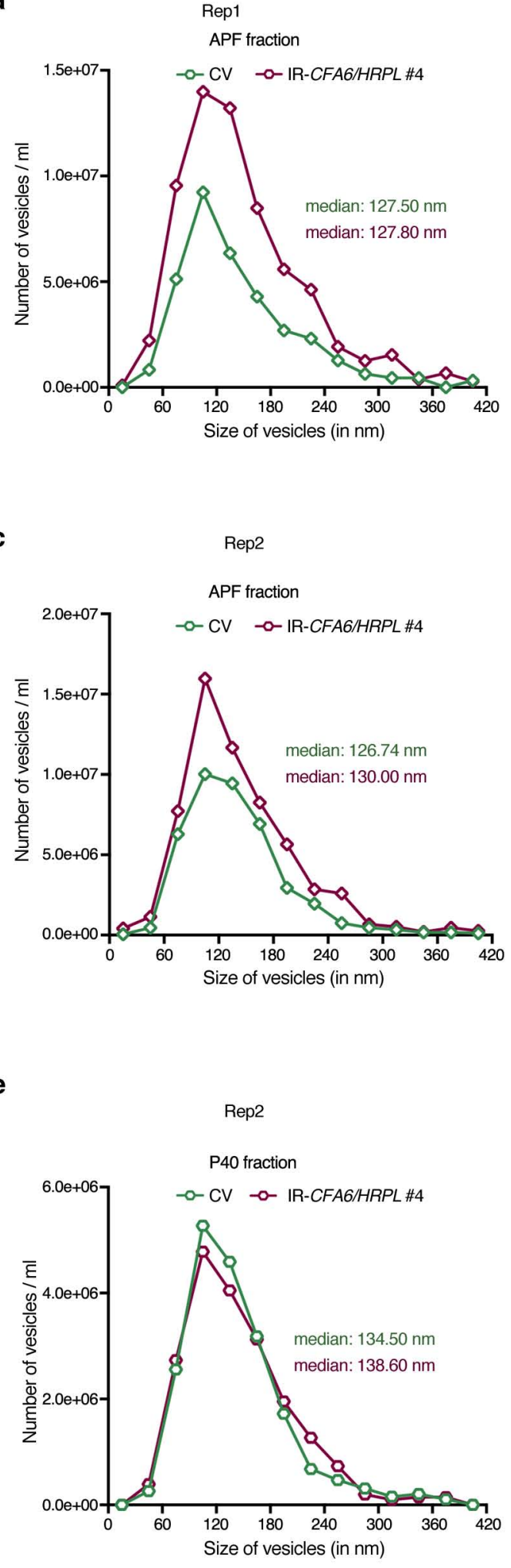

b

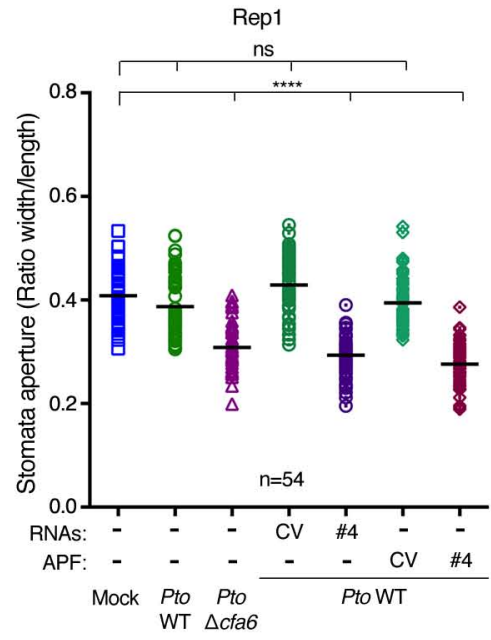

d

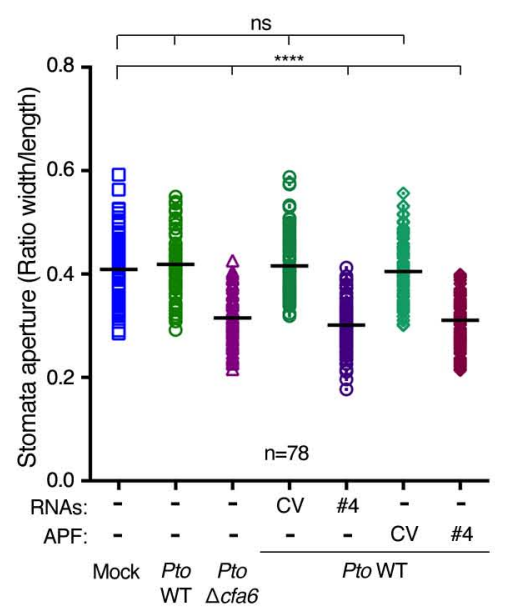

f

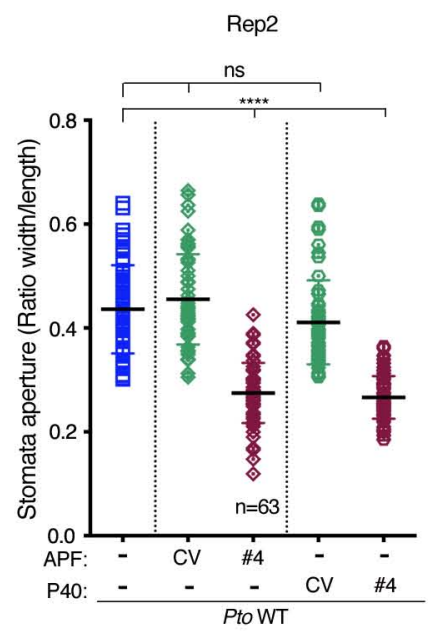

Fig. S10. Apoplastic fluid and P40 vesicles isolated from Arabidopsis IR-CFA6/HRPL leaves trigger suppression of Pto DC3000-induced stomatal reopening a. \& c. Apoplastic EVs from the Arabidopsis transgenic lines IR-CYP51 (CV) and IR-CFA6/HRPL\#4 (\#4) were isolated by collecting the apoplastic fluids (APF) from these plants and analysed using Nanoparticle Tracking Analysis (NTA). The number of particles, the size of particles according to their diameter (in nm), and the median size of the particles are depicted for each sample from two independent replicates. b. \& d. The ability of Pto WT to reopen stomata was altered to similar levels upon exogenous application of APF as compared to total RNAs derived from IR-CFA6/HRPL\#4 plants. Stomata aperture was measured in Col-0 leaves incubated for 1 hour with water (Mock) or total

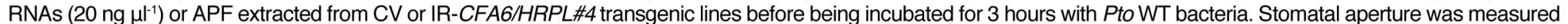
and analyzed as described previously. e. Additional biological replicate from Fig. 8a: Apoplastic EVs from the Arabidopsis transgenic lines IR-CYP51 (CV) and IRCFA6/HRPL\#4 (\#4) were isolated by collecting the apoplastic fluids (APF) and subjected them to ultracentrifugation at $40,000 \mathrm{~g}$ (P40) as previously described (ref). The population of particles from the recovered P40 fractions were analysed using Nanoparticle Tracking Analysis (NTA). The number of particles, the size of particles according to their diameter (in $\mathrm{nm}$ ), and the median size of the particles are depicted for each sample. f. Additional biological replicate from Fig. 8b: Both the APF and the P40 fractions triggered full suppression of stomatal reopening. Stomatal aperture was measured in Col-0 leaves that were incubated for 1 hour with the above APF or P40 fractions before being inoculated with Pto WT for 3 hours. Stomatal aperture was measured and analyzed as described previously. For all the stomata experiments, $n=$ number of stomata analyzed per condition and statistical significance was assessed using the ANOVA test (ns: $p$-value $>0.05$; ${ }^{* * * *}$ : $p$-value $<0.0001$ ). 


\section{Supplementary Table legend}

Supplementary Table 1. Targets of 500 most abundant siRNAs specific to $c f a 6$ or $h r p L$ derived from IR-CFA6/HRPL in the Pto DC3000 genome

Supplementary Table 2. Targets of 500 most abundant siRNAs specific to cfa6 or hrpL derived from IR-CFA6/HRPL in the Arabidopsis thaliana Col-0 genome

Supplementary Table 3. Targets of 500 most abundant anti- $h r p L$ siRNAs derived from IR$C F A 6 / H R P L$ in the WT or mutated $h r p L$ versions

Supplementary Table 4. List of primer sequences used in this study 


\section{SRNA_souıGenome ID}

cfa6 Pst DC3000 NC_004578.1_cds_NP_794435.1_4579

cfa6

cfa6

cfa6

cfa6

fa6

cfa6

fa6

cfa6

hrpL

hrpL

hrpL Pst DC3000 NC_004578.1_cds_NP_790362.1 499

Pst DC3000 NC_004578.1_cds_NP_791928.1_2070 Pst DC3000 NC_004578.1_cds_NP_791231.1_1369 Pst DC3000 NC_004578.1_cds_NP_790641.1_777 Pst DC3000 NC_004578.1_cds_NP_795316.1_5464 Pst DC3000 NC_004578.1_cds_NP_794924.1_5072 Pst DC3000 NC_004578.1_cds_NP_791957.1_2100 Pst DC3000 NC_004578.1_cds_NP_791186.1_1324 Pst DC3000 NC 004578.1 cds_NP 791667.1_1809 Pst DC3000 NC_004578.1_cds_NP_793378.1_3523 Pst DC3000 NC_004578.1_cds_NP_791231.1_1369 Pst DC3000 NC_004578.1_cds_NP_794435.1_4579 Pst DC3000 NC_004578.1_cds_NP_793956.1_4100 Pst DC3000 NC_004578.1_cds_NP_793436.1_3579
Targeted.by. (Unique reads) Targeted.by (Total rea lowest_e_valı locus

$7075728 \quad 0,0000258$ gene $=$ cfa 6

0,17 gene $=$ pqq $F$

description

coronafacic acid polyketide synthase I

720356

117477

77391

68426

10724

8838

0,17 locus_tag=

057 gene=hrpL

0,51 locus_tag $=P S$
1,5 gene $=$ atpC

Pynthesis protein $F$

1,5

pyoverdine chromophore precursor synthetase

0,51 locus

1,5 locus tag=PSPTO 1843 aspartate kinase

0,51 locus_tag=PSPTO_3599 hypothetical protein

3478
2781

1548

6698293

11897

0,0000258 gene $=$ hrpL

0,019 gene $=$ cfa 6

0,51 locus tag=PSPTO_4195 hypothetical protein

1641

1,5 locus_tag=PSPTO 3662 xanthine dehydrogenase accessory factor $\mathrm{XdhC}$ 
SRNA_source Genome ID

Alh TAIR10 NC_003076.8_cds_NP_001032042.1_4462 Ath TAIR10 NC_003076.8_cds_NP_197110.1_39886

Ath TAIR10 NC 0030768 dds NP 001032057.1 $4507 \mathrm{C}$

Ath TAIR10 NC_003071.7_cds_NP_001031385.1_1506

Ath TAIR10 NC_003074.8_cds_NP_190127.1_26372

Ath TAIR10 NC_003075.7_cds_NP_192119.2_30164

Ath TAIR10 NC_003076.8_cds_NP_974885.1_43802

Ath TAIR10 NC_003070.9_cds_NP_171667.11137

Ath TAR10 NC_003076__d__N_001320772.1_8776

Ath TAIR10 NC 003074.8cds_NP-191458.1 28849

Ath TAIR10 NC 037304.1 cds YP 009472115248160

Ath TAIR10 NC_003070.9_cds_NP_173075.3_3102

Ath TAIR10 NC_003071.7_cds_NP_179907.1_15421

Ath TAIR10 NC_003074.8_cds_NP_001030896.1_2900

Ath TAlR10 NC_003070.9_cds_NP_849898.1_11877

Ath TAIR10 NC_003074.8_cds_NP_188618.3_23966

Ath TAIR10 NC 003076 _cds_N- 196827.1

Ath TAIR10 NC 003075.7 d ds NP_001328069.1 35815

Ath TAIR10 NC 003074.8 cds NP 191259.128493

Ath TAIR10 NC_003074.8_cds_NP_191274.1_28522

Ath TAIR10 NC_003076.8_cds_NP_198474.1_42307

Ath TAIR10 NC_003074.8_cds_NP_001118685.1_24672

Ath TAIR10 NC_003070.9_cds_NP_177125.3_10577

Ath TAIR10 NC_003076.8_cds_NP_001332094.1_4301

Ath TAIR10 NC 003075.

Ath TAIR10 NC 003076.8 cds_NP 201271.1.47530

Ath TAIR10 NC_003075.7 dds_NP_001328078.1_34834

Ath TAIR10 NC_003076.8_cds_NP_680446.1446045

Ath TAIR10 NC_003076.8_cds_NP_195740.1_37189

Ath TAlR10 NC_003074.8_cds_NP_191052.2_28089

Ath TAIR10 NC_003076.8_cds_NP_196750.1_3918

Ath TAIR10 NC_003075.7_cds_NP_192855.1_31308

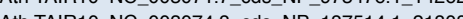

Ath TAIR10 NC 003075.

Ath TAIR10 NC 003071.7_cds_NP_178535.3_13300

Ath TAIR10 NC_003074.8_cds_NP_188705.1_24146

Ath TAIR10 NC_003074.8_cds_NP_001078165.1_23419

Ath TAIR10 NC_003070.9_cds_NP_178014.2_12198

Ath TAIR10 NC_003076.8_cds_NP_196118.1_37890

Ath TAIR10 NC_003071.7_cds_NP_181050.1_17673

Ath TAIR10 NC_003074.8_ds_NP_50617.1_25386

Ath TAIR10 NC 0030768 cdd NP 001190624144774

Ath TAIR10 NC 0030748 cds NP 190058.126252

Ath TAIR10 NC_003074.8_cds_NP_188938.1_24538

Ath TAIR10 NC_003070.9_cds_NP_001321840.1_4928

Ath TAIR10 NC_003076.8_cds_NP_196331.2_38305

Ath TAIR10 NC_003076.8_cds_NP_201215.1_47418

Ath TAIR10 NC_003074.8_cds_NP_001189846.1_21882

Ath TAIR10 NC_003074.8_cds_NP_189335.2_25302

Ath TAIR10 NC_003070._cds_NP_849573.1_124
Targeted.by. (Unique reads) Targeted.by (Total reads) lowest_e_value locus

1,9 gene $=\mathrm{OVA} 2$

description

, I L I, M and V) family protein

5.7 locus tag=AT5G16060 Cytochrome c oxidase biogenesis protein $\mathrm{Cmc1}$-like protein

946535

278379

197128

186313

175169

174998
158983
145895

116106

869230

69276

68649
67561

67561
59612

55911

47805

45371

37320

34049

24103

23034

20963

18650

18268

17244

15932
14056

13338

11429

5,7 gene=TPS (n)

5,7 locus tag=AT2G21300 ATP binding microtubule motor family protein

5,7 locus_tag=AT3G45410 Concanavalin A-like lectin protein kinase family protein

17 locus_lag=AT4G02100 Heal shock prolein DnaJ with tetraticopeplide repeat-containing protein 1,9 gene=OSB3 $\quad$ organellar single-stranded DNA binding protein 3

$\begin{array}{ll}5,7 \text { gene=GPAT4 } & \text { glycerol-3-p } \\ 17 \text { gene=ARL2 } & \text { ARG1-like } 2\end{array}$

17 gene=ARL2

17 gene=IPMI1

isopropylmalate isomerase 1

perfamily protein

17 gene=UGT84B1 UDP-glucosyl transferase 84B1

5,7 gene=SAC9 sacl homology domain-containing protein $/ \mathrm{WW}$ domain-containing protein

Uncharacterized protein family UPFO090

1,9 gene=PRP40C pre-mRNA-processing

17 locus tag=AT5G13230 Tetratricopeptide repeat (TPR)-like superfamily protein

5,7 gene=EDA39 calmodulin-binding family protein

5,7 gene=NAP57 homologue of NAP57

Protein phosphatase $2 \mathrm{C}$ family protein

AT1 G08760 GPI-anchered adhesin-like protein, putative (DUF936)

17 gene=UGT84A1 UDP-Glycosyltransferase superfamily protein

17 locus tag=AT4G28150 hypo MET, PRO, and GLY protein 2

17 locus tag=AT5G56890 Protein kinase superfamily proten

17 locus_tag=AT5G01200 Duplicated homeodomain-like superfamily protein

1,9 gene=PMR6

Pectin lyase-like superfamily protein

5,7 gene=EMB3135

harpin-induced protei

17 locus_tag=AT4G11170 Disease resistance protein (TIR-NBS-LRR class) family

5,7 locus_tag=AT2G16980 Major racilitator superfamily protein

17 gene-RIC7 17020 a

17 gene=LpXB transferases, transferring glycosyl groups

17 locus tag=AT3G20690 F-box and associated interaction domains-containing protein

5,7 gene=LPD1 lipoamide dehydrogenase 1

17 locus_tag=AT1G78930 Mitochondrial transcription termination factor family protein

$\begin{array}{ll}17 \text { gene=SUN1 } & \text { SAD1/UNC-84 domain protein } 1 \\ 17 \text { locus_tag=AT2G35050 kinase superfamily with octicosapeptide/Phox/Bem1p domain-containing protein }\end{array}$

SAD1/UNC-84 domain protein 1

17 locus_lag=AT_G25430 Polynucleotidyl transferase, ribonuclease $\mathrm{H}$-like superfamily prote

17 gene=BAM1
1,9 gene=ADT4

in kinase family protein

9 logenate dehydratase 4

5,7 gene=NRT1. 6 nitrate transporter 1.6

17 locus_tag=AT5G07140 Protein kinase superfamily prote

1,9 locus_tag=AT5G64100 Peroxidase superfamily proten

17 gene=PLL3

pol-like 3

17 locus_tag=AT3G26990 ENTH/NHS family protein

5,7 locus_tag=AT1G01540 Protein kinase superfamily protein 
Ath TAIR10 NC_003076.8_cds_NP_199754.2_44698 Ath TAlR10 NC_003074.8_cds_NP_001190123.1_28699 Ath TAIR10 NC 0030748 cds NP 187718.1_22005 Ath TAIR10 NC 003070.9 cds NP 564584. 7408 Ath TAIR10 NC_003071.7_cds_NP_180929.1_17431 Ath TAIR10 NC_003074.8_cds_NP_188014.1_22820 Ath TAIR10 NC_003074.8_Cds_NP_566579.1_23509 Ath TAlR10 NC_003076.8_cds_NP_196567.2_38816 Ath TAIR10 NC_003074.8_cds_NP_566381.1_22144
Ath TAIR10 NC_0030757_cds_NP001328421.1_3471 Ath TAR10 NC 003071.7_ds_NP_179618.1_14323 Ath TAIR10 NC 003074.8 cds NP_001189932.123836 Ath TAIR10 NC 003075.7 cds NP 567998.336468 Ath TAIR10 NC_003076.8_cds_NP_200190.1_45491 Ath TAIR10 NC_003075.7_cds_NP_193070.1_31729 Ath TAIR10 NC_003075.7_cds_NP_195043.1_35837 Ath TAIR10 NC_003076.8_cds_NP_200667.2446366 Ath TAIR10 NC_003075.7_cds_NP_001327989.1_3586 Ath TAIR10 NC 003070. cds NP 171980.1780 Ath TAIR10 NC 003070 cds NP 0013190351355 Ath TAIR10 NC_003076.8_cds_NP_198624.1.42592 Ath TAIR10 NC_003076.8_cds_NP_001331990.14602 Ath TAIR10 NC_003076.8_cds_NP_198101.2_41844 Ath TAIR10 NC_003074.8_cds_NP_001326732.1_27241 Ath TAIR10 NC_003074.8_cds_NP_188706.1_24147 Ath TAIR10 NC_003074.8_cds_NP_187423.2_21619 Ath TAIR10 NC 003070.9_cds NP_0013205781_12038 Ath TAIR10 NC 003074.8 cds NP_ 191318.4 28609 Ath TAIR10 NC_003071.7 cds NP 565351.1 13749 Ath TAIR10 NC_003076.8_cds_NP_196819.1_39336 Ath TAIR10 NC_003076.8_cds_NP_197841.1_41358 Ath TAIR10 NC_003074.8_cds_NP_001325725.1_22115 Ath TAIR10 NC_003071.7_cds_NP_973476.1_14262 Ath TAIR10 NC_003076.8_cds_NP_001119157.1_37478 Ath TAIR10 NC_003076 8_cds_NP_199757.1_4702 Ath TAIR10 NC 0030757 cds NP 195464236670 Ath TAIR10 NC_003075.7_cds_NP_193506.2 32749 Ath TAIR10 NC 003074.8 cds NP 001326496.122571 Ath TAIR10 NC_003076.8_cds_NP_201169.1_47342 Ath TAIR1O NC_003071.7_cds_NP_001324812.1_18586 Ath TAIR10 NC_003076.8_dds_NP_001330670.1_4332 Ath TAIR10 NC_003076.8_cds_NP_200784.2_46584 Ath TAIR10 NC 003075 . deds NP 567330.1 Ath TAIR10 NC 0030748 ds NP 5671181 Ath TAIR10 NC 003074.8 cds NP 001327831.120318 Ath TAIR10 NC_003070.9_cds_NP_001321735.13199 Ath TAIR10 NC_003076.8_cds_NP_200382.1_45830 Ath TAIR10 NC_003074.8_cds_NP_001189790.1_20428 Ath TAIR10 NC_003076.8_cds_NP_001332517.1_4474 Ath TAIR10 NC_003076.8_cds_NP_001330525.1_4294 Ath TAlR10 NC_003076.8_dds_NP_199253.1_43717 Ath TAIR10 NC_003076.8_cds NP_198103___41847

\begin{tabular}{|c|c|c|}
\hline 5007 & 17 locus_tag=AT5G49430 & eat and Bromo-WDR9--like domain-containing protein \\
\hline 4966 & 17 locus_tag=AT3G58110 & hypothetical protein \\
\hline 4921 & 17 gene=GulLO7 & FAD-dependent oxidoreductase family protein \\
\hline 4920 & 17 locus_tag=AT3G11070 & Outer membrane OMP85 family protein \\
\hline 4854 & 17 gene= $=$ CN & Protein kinase superfamily protein \\
\hline 4600 & 17 locus_tag=AT2G33730 & P-loop containing nucleoside triphosphate hydrolases superfamily protein \\
\hline 4232 & 17 locus_tag=AT3G13980 & SKIDACH domain protein \\
\hline 4095 & 17 gene=RPL3P & ribosomal protein L3 plastid \\
\hline 3861 & 5,7 locus_tag=AT5G 10050 & NAD(P)-binding Rossmann-fold superfamily protein \\
\hline 3613 & 17 locus_tag=AT3G10770 & Single-stranded nucleic acid binding R3H protein \\
\hline 3478 & 17 locus_tag=AT4G27595 & WEB family protein (DUF827) \\
\hline 3411 & 5,7 locus_tag=AT2G20280 & Zinc finger $\mathrm{C}-\mathrm{x} 8-\mathrm{C}-\mathrm{x} 5-\mathrm{C}-\mathrm{x} 3-\mathrm{H}$ type family protein \\
\hline 3310 & 17 gene=PREP1 & presequence protease 1 \\
\hline 3209 & 5,7 locus_tag=AT4G36190 & Serine carboxypeptidase S28 family protein \\
\hline 3063 & 5,7 locus_tag=AT5G53790 & F-box protein, putative (DUF295) \\
\hline 2856 & 17 gene=LRX3 & Leucine-rich repeat (LRR) family protein \\
\hline 2854 & 1,9 locus_tag=AT4G33170 & Tetratricopeptide repeat (TPR)-like superfamily protein \\
\hline 2781 & 17 gene=RANBP 1 & RAN binding protein 1 \\
\hline 2608 & 5,7 locus_tag=AT4G33280 & AP2/B3-like transcriptional factor family protein \\
\hline 1309 & 5,7 locus_tag=AT5G58950 & Protein kinase superfamily protein \\
\hline 976729 & 5,7 locus_tag=AT1G04880 & HMG (high mobility group) box protein with ARID/BRIGHT DNA-binding domain-containing proteir \\
\hline 829463 & 5,7 locus_tag=AT1G18660 & zinc finger (C3HC4-type RING finger) family protein \\
\hline 688488 & 5,7 locus_tag=AT5G38080 & transmembrane protein \\
\hline 397983 & 17 gene=HAG2 & histone acetyltransferase of the GNAT family 2 \\
\hline 344917 & 1,9 locus_tag=AT5G 27490 & Integral membrane Yip 1 family protein \\
\hline 253780 & 5,7 locus_tag=AT3G50380 & vacuolar protein sorting-associated protein, putative (DUF1162) \\
\hline 200098 & 1,9 locus_tag=AT3G20700 & F-box associated ubiquitination effector family protein \\
\hline 175936 & 0,071 locus_tag=AT3G07660 & flocculation protein (DUF1296) \\
\hline 129003 & 17 locus_tag=AT5G59790 & UPSTREAM OF FLC protein (DUF966) \\
\hline 112138 & 17 locus_tag=AT1G79103 & 3 hypothetical protein \\
\hline 108905 & 5,7 locus_tag=AT3G57590 & F-box and associated interaction domains-containing protein \\
\hline 105824 & 17 gene=CRCK3 & calmodulin-binding receptor-like cytoplasmic kinase 3 \\
\hline 102855 & 1,9 gene $=\mathrm{EXO} 70 \mathrm{C} 1$ & exocyst subunit exo70 family protein $\mathrm{C} 1$ \\
\hline 100643 & 5,7 gene=DMR6 & 2-oxoglutarate $(2 \mathrm{OG})$ and $\mathrm{Fe}(\mathrm{II})$-dependent oxygenase superfamily protein \\
\hline 96366 & 1,9 gene=CAT7 & cationic amino acid transporter 7 \\
\hline 87960 & 5,7 locus_tag=AT2G16980 & Major facilitator superfamily protein \\
\hline 85801 & 5,7 gene=GSTL 1 & glutathione transferase lambda 1 \\
\hline 83985 & 5,7 gene=GRP3S & glycine-rich protein 3 short isoform \\
\hline 83790 & 17 gene=ACLB-2 & ATP citrate lyase subunit B 2 \\
\hline 75027 & 5,7 locus_tag=AT4G 37480 & Chaperone DnaJ-domain superfamily protein \\
\hline 71605 & 1,9 gene=HDG4 & homeodomain GLABROUS 4 \\
\hline 62061 & 5,7 gene $=$ HAC5 & histone acetyltransferase of the CBP family 5 \\
\hline 57212 & 17 locus_tag=AT5G63640 & ENTH/VHS/GAT family protein \\
\hline 55676 & 5,7 gene=DAR2 & DA1-related protein 2 \\
\hline 54997 & 17 gene=SDG25 & SET domain protein 25 \\
\hline 50514 & 17 locus_tag=AT5G59760 & hypothetical protein (DUF1635) \\
\hline 46678 & 5,7 locus_tag=AT2G41730 & calcium-binding site protein \\
\hline 44978 & 5,7 gene=ACS11 & 1-aminocyclopropane-1-carboxylate synthase 11 \\
\hline 44216 & 5,7 locus_tag=AT3G61790 & Protein with RING/U-box and TRAF-like domain \\
\hline 39036 & 1,9 locus_tag=AT3G01310 & Phosphoglycerate mutase-like family protein \\
\hline 37902 & 5,7 gene $=\mathrm{HAC} 12$ & histone acetyltransferase of the CBP family 12 \\
\hline 37854 & 17 gene=Tic20-V & TIC 20-v-like protein \\
\hline 37489 & 17 locus_tag=AT3G018 & EEIG1/EHBP1 protein amino-terminal domain protein \\
\hline 31145 & 1,9 gene=FRO 6 & ferric reduction oxidase 6 \\
\hline 26051 & 1,9 gene=MYB24 & myb domain protein 24 \\
\hline 25564 & 17 locus_tag=AT5G44400 & FAD-binding Berberine family protein \\
\hline 24115 & 1,9 gene=ОСТ3 & organic cation/carnitine transporter 3 \\
\hline & (20) & \\
\hline
\end{tabular}


Ath TAIR10 NC_003076.8_cds_NP_201234.1_47447 Ath TAlR10 NC_003076.8_cds_NP_197761.2_41187 Ath TAIR10 NC 003076.8 cds NP_001331276.145111 Ath TAIR10 NC 003074.8 cds NP 191310.1 28593 Ath TAIR10 NC_003071.7_cds_NP_973714.1_20207 Ath TAlR10 NC_003070.9_cds_NP_564401.1_5865 Ath TAIR10 NC_003075.7_cds_NP_001031658.1_32777 Ath TAIR10 NC_003076.8_cds_NP_199575.1_44334 Ath TAIR10 NC_003076.8_cds_NP_196554.1_38789 Ath TAIR10 NC 0030757 dods NP-192500.13081 Ath TAIR10 NC_003070.9_cds_NP_565212.1 12310 Ath TAIR10 NC 0030748 cds NP 001030659.121618 Ath TAIR10 NC_003076.8_cds_NP_974747.1_38321 Ath TAlR10 NC_003070.9_cds_NP_176638.1_9648 Ath TAIR10 NC_003076.8_cds_NP_200359.1_45771 Ath TAIR10 NC_003070.9_cds_NP_001322007.1_1492 Ath TAIR10 NC_003076.8_cds_NP_568718.1_44790
Ath TAIR10 NC0030768_cds_NP_0013320941_4301 Ath TAIR10 NC 0030708 cds NP 197483.140559 Ath TAIR10 NC 003074.8 cds NP 191069.2 28130 Ath TAIR10 NC 003076.8 cds NP 001331299.1 41301 Ath TAIR10 NC_003074.8_cds_NP_191697.1_29312 Ath TAlR10 NC_003070.9_cds_NP_001031225.1_9550 Ath TAIR10 NC_003076.8_Cds_NP_568958.1_47160 Ath TAIR10 NC_003074.8_cds_NP_187170.2_21094 Ath TAlR10 NC_003076.8_cds_NP_568711.1_447721 Ath TAIR10 NC 003071.7 Cds_NP 001324747.1 Ath TAIR10 NC_003070.9 cds_NP_001322409.15083 Ath TAIR10 NC_003071.7 cds_NP_001031477.1_17537 Ath TAIR10 NC_003076.8_cds_NP_001154791.1_47159 Ath TAIR10 NC_003076.8_cds_NP_199956.1_45049 Ath TAlR10 NC_003075.7_cds_NP_567467.1_32247 Ath TAlR10 NC_003070.9_ds_NP_174229.2_5312 Ath TAIR10 NC_003070.9_cds_NP_174122.1_5100 Ath TAIR10 NC003075. Cds_NP001031681.13330 Ath TAIR10 NC 003075.7 cds NP 194594.1_34924 Ath TAIR10 NC 003076.8 cds NP 001332397.14078 Ath TAIR10 NC_003075.7_cds_NP_191979.2_29874 Ath TAIR10 NC_003071.7_cds_NP_001323677.1_1474 Ath TAlR10 NC_003071.7_Cds_NP_179497.1_14629 Ath TAIR10 NC_003075.7_cds_NP_567440.1_32031 Ath TAlR10 NC_0030076.8_cds_NP_199024.1_43277 Ath TAIR10 NC 000071_. Ath TAIR10 NC 003070.9 cds NP 001322651.18127 Ath TAIR10 NC 003075.7 cds NP 193348.132368 Ath TAIR10 NC_003071.7_cds_NP_182268.1_20114 Ath TAIR10 NC_003074.8_cds_NP_187914.1_22603 Ath TAIR10 NC_003076.8_cds_NP_001318654.1_4162 Ath TAIR10 NC_003075.7_Cds_NP_192982.1_31527 Ath TAIR10 NC_003075.7_cds_NP_193381.1_32446 Ath TA1R10 NC.003070__ds_NP_ Ath TAIR10 NC_003076.8_cds_NP_197528_1_40653

\begin{tabular}{|c|c|c|}
\hline $\begin{array}{l}22704 \\
22682\end{array}$ & $\begin{array}{l}5,7 \text { gene=DIT2.1 } \\
17 \text { gene=PHS1 }\end{array}$ & $\begin{array}{l}\text { dicarboxylate transport } 2.1 \\
\text { dual specificity protein phosphatase family protein }\end{array}$ \\
\hline 21619 & 5,7 locus_tag=AT1G06050 & ENHANCED DISEASE RESISTANCE-like protein (DUF1336) \\
\hline 19898 & 17 gene=AHG1 & Protein phosphatase $2 \mathrm{C}$ family protein \\
\hline 19242 & 5,7 gene=ADPG1 & Pectin lyase-like superfamily protein \\
\hline 17874 & 1,9 locus_tag=AT2G47970 & Nuclear pore localization protein NPL4 \\
\hline 17376 & 5,7 locus_tag=AT1G32415 & pentatricopeptide (PPR) repeat-containing protein \\
\hline 17272 & 5,7 gene=AGD8 & ARF-GAP domain 8 \\
\hline 16947 & 5,7 gene=NF-YB2 & nuclear factor $\mathrm{Y}$, subunit $\mathrm{B} 2$ \\
\hline 16506 & 5,7 gene=NRPB4 & RNA polymerase II, Rpb4, core protein \\
\hline 15931 & 5,7 locus_tag=AT1G71110 & transmembrane protein \\
\hline 15878 & 5,7 gene=MEKK 1 & MAPK/ERK kinase kinase 1 \\
\hline 15850 & 0,64 gene=FTSH12 & FTSH protease 12 \\
\hline 15616 & 1,9 gene=COL9 & CONSTANS-like 9 \\
\hline 15454 & 17 locus_tag=AT5G07225 & RING/U-box superfamily protein \\
\hline 14842 & 0,64 gene=DUO3 & Homeodomain-like superfamily protein \\
\hline 14745 & 17 gene=SVL1 & SHV3-like 1 \\
\hline 13961 & 5,7 gene=MOM & ATP-dependent helicase family protein \\
\hline 13773 & 17 gene=AFB5 & auxin F-box protein 5 \\
\hline 11897 & 5,7 gene=SDH2-2 & succinate dehydrogenase 2-2 \\
\hline 11891 & 17 gene=emb2734 & ARM repeat superfamily protein \\
\hline 11879 & 5,7 gene=ABCG16 & ABC-2 type transporter family protein \\
\hline 11792 & 1,9 locus_tag=AT5G24275 & En/Spm-like transposon \\
\hline 11623 & 5,7 gene=TRM14 & Phosphatidylinositol N-acetyglucosaminlytransferase subunit P-like protein \\
\hline 11566 & 17 locus_tag=AT1G63980 & D111/G-patch domain-containing protein \\
\hline 11047 & 1,9 locus_tag=AT5G62650 & Tic22-like family protein \\
\hline 10716 & 1,9 locus_tag=AT3G05190 & D-aminoacid aminotransferase-like PLP-dependent enzymes superfamily protein \\
\hline 10321 & 1,9 gene=BLOS2 & biogenesis of Iysosome organelles complex 1 subunit-like protein \\
\hline 10309 & 17 gene $=\mathrm{GDH} 1$ & glutamate dehydrogenase 1 \\
\hline 9085 & 5,7 locus_tag=AT2G03140 & alpha/beta-Hydrolases superfamily protein \\
\hline 9073 & 1,9 locus_tag=AT1G27900 & RNA helicase family protein \\
\hline 8951 & 17 locus_tag=AT2G34300 & S-adenosyl-L-methionine-dependent methyltransferases superfamily protein \\
\hline 8875 & 5,7 gene=ELF5 & proline-rich family protein \\
\hline 8729 & 5,7 gene $=\mathrm{EYE}$ & conserved oligomeric Golgi complex component-related / COG complex component-like protein \\
\hline 8652 & 17 locus_tag=AT4G15475 & F-box/RNI-like superfamily protein \\
\hline 8503 & 17 locus_tag=AT1G29350 & RNA polymerase II degradation factor-like protein (DUF1296) \\
\hline 7703 & 5,7 gene=ABCB14 & P-glycoprotein 14 \\
\hline 7408 & 0,64 gene=MUR3 & Exostosin family protein \\
\hline 7198 & 5,7 gene=JMJ14 & JUMONJI 14 \\
\hline 7192 & 5,7 locus_tag=AT4G28650 & Leucine-rich repeat transmembrane protein kinase family protein \\
\hline 7176 & $17 \mathrm{gene}=\mathrm{B} 73$ & molybdopterin biosynthesis CNX1 protein / molybdenum cofactor biosynthesis enzyme CNX1 (CNX1) \\
\hline 7085 & 5,7 locus_tag=AT4G00700 & C2 calcium/lipid-binding plant phosphoribosyltransferase family protein \\
\hline 7061 & 5,7 locus_tag=AT2G19890 & hypothetical protein \\
\hline 6367 & 5,7 gene $=\mathrm{SHT}$ & spermidine hydroxycinnamoyl transferase \\
\hline 6041 & 17 gene=ORC1A & origin recognition complex 1 \\
\hline 6017 & 5,7 locus_tag=AT5G42090 & Lung seven transmembrane receptor family protein \\
\hline 5741 & 17 gene=RPN1A & $26 \mathrm{~S}$ proteasome regulatory subunit S2 $1 \mathrm{~A}$ \\
\hline 4870 & 5,7 locus_tag=AT2G36885 & translation initiation factor \\
\hline 4826 & 17 locus_tag=AT1G54930 & GRF zinc finger / Zinc knuckle protein \\
\hline 4616 & 1,9 gene=ARA 1 & arabinose kinase \\
\hline 4447 & 17 gene=MYB12 & myb domain protein 12 \\
\hline 4406 & 0,64 locus_tag=AT3G13070 & CBS domain-containing protein / transporter associated domain-containing protein \\
\hline 3844 & 5,7 locus_tag=AT5G26090 & Plant self-incompatibility protein S1 family \\
\hline 3665 & 5,7 locus_tag=AT4G12450 & zinc finger ( $\mathrm{C} 2 \mathrm{H} 2$ type) family protein \\
\hline 3515 & 17 gene=INT4 & inositol transporter 4 \\
\hline 3346 & 5,7 locus_tag=AT1G27470 & transducin family protein / WD-40 repeat family protein \\
\hline 3056 & 17 locus_tag=AT5G48440 & FAD-dependent oxidoreductase family protein \\
\hline 2937 & 5,7 gene=SPS1F & sucrose phosphate synthase $1 \mathrm{~F}$ \\
\hline
\end{tabular}


Ath TAIR10 NC 003071. Cds NP 180277.116154

Ath TAIR10 NC_003075.7 cds NP 195338.1 36462

Ath TAIR10 NC_003074.8_cds_NP_566552.2_23337

Ath TAlR10 NC_003074.8_cds_NP_189192.1_25015

Ath TAIR10 NC_003070.9_cds_NP_001321631.1_3315

Ath TAIR10 NC_003070.9_cds_NP_001321597.1_12311

Ath TAlR10 NC_003071.7_cds_NP_180545.2_16644

At TAR10 NC 0030717 cds NP 001324140.113200

Ath TAIR10 NC_003074.8 cds_NP_191355.1 28674

Ath TAIR10 NC_003076.8_cds_NP 851188.1_45571

Ath TAIR10 NC_003071.7_cds_NP_180198.1_15999

Ath TAIR10 NC_003070.9_cds_NP_001185169.1_6950

Ath TAIR10 NC_003070.9_cds_NP_564824.1_9576

Ath TAlR10 NC_003071.7_cds_NP_565297.1_13067

Ath TAlR10 NC_003075.7_cds_NP_974490.1_29835
5,7 gene=TBL23 TRICHOME BIREFRINGENCE-LIKE 23

5,7 locus_tag=AT5G67610 transmembrane protein (DUF2215)

1,9 locus_tag=AT2G27090 bZIP transcription factor (DUF630 and DUF632)

7 locus tag=AT4G36150 Disease resistance protein (TIR-NBS-LRR class) family

, 9 gene=DEG12 protease Do-like protein

5,8 locus_tag=AT3G25640 MIZU-KUSSEI-like protein (Protein of unknown function, DUF617)

5,7 gene=UBC34 $\quad$ ubiquitin-conjugating enzyme 34

5,8 gene=FTSH 12 FTSH protease 12

1,9 locus_tag=AT2G29840 RING/U-box superfamily proten

17 1 yb domain protein 119

57 locus tag=AT3G57960 Emsy N

17 gene=CNGC4 cyclic nucleotide-gated cation channel 4

5,7 gene=GP ALPHA $1 \quad$ G protein alpha subunit 1

17 gene=AGO1 Stabilizer of iron transporter SufD / Polynucleotidyl transferase

5,7 gene=DAA1 P-loop containing nucleoside triphosphate hydrolases superfamily protein

1,9 gene $=$ SK 16

17 locus_tag=AT4G00500 alpha/beta-Hydrolases superfamily protein 


\begin{tabular}{|c|c|c|c|c|c|}
\hline sRNA_source & Target & Read & Read_abundance (total count) & Blast.e.value & Pairing_energy (Kcal/mol) \\
\hline hrpL & WT hrpL & CAAGAAACACGCACTGGAGAA & 567546 & 0,000000173 & $-36,59$ \\
\hline hrpL & WT hrpL & CAGCCAGGTCTGCGGTTTGCT & 566924 & 0,000000173 & $-41,6$ \\
\hline hrpL & WT hrpL & ACACGCACTGGAGAATGTCAT & 353690 & 0,000000173 & $-35,81$ \\
\hline hrpL & WT hrpL & AGCAAACCGCAGACCTGGCTG & 253785 & 0,000000173 & $-40,21$ \\
\hline hrpL & WT hrpL & TTCTCCAGTGCGTGTTTCTTG & 229178 & 0,000000173 & $-36,55$ \\
\hline hrpL & WT hrpL & GATGCCACACAGCCAGGTCTG & 211021 & 0,000000173 & $-41,59$ \\
\hline hrpL & WT hrpL & CGGATCAGGTTCAGCGCGATG & 167512 & 0,000000173 & $-41,42$ \\
\hline hrpL & WT hrpL & ATGACATTCTCCAGTGCGTGT & 143887 & 0,000000173 & $-37,02$ \\
\hline hrpL & WT hrpL & ACAGCCAGGTCTGCGGTTTGCT & 107864 & $6,36 \mathrm{E}-08$ & $-42,25$ \\
\hline hrpL & WT hrpL & CAGCTCGGAATGCACTTCGTC & 106286 & 0,000000173 & $-39,24$ \\
\hline hrpL & WT hrpL & GACGAAGTGCATTCCGAGCTG & 105824 & 0,000000173 & $-38,7$ \\
\hline hrpL & WT hrpL & CTGACGATACATTTTGGGGAA & 97869 & 0,000000173 & $-33,83$ \\
\hline hrpL & WT hrpL & TTCCGCAAAATGTATCGTCAG & 97203 & 0,000000173 & $-31,34$ \\
\hline hrpL & WT hrpL & CAGACCTGGCTGTGTGGCATC & 94841 & 0,000000173 & $-42,49$ \\
\hline hrpL & WT hrpL & TTTCCTGATACGGCTGACGAT & 86176 & 0,000000173 & $-35,92$ \\
\hline hrpL & WT hrpL & ATCGTCAGCCGTATCAGGAAA & 85934 & 0,000000173 & $-36,62$ \\
\hline hrpL & WT hrpL & CTGGGAAGACGAAGTGCATTC & 74216 & 0,000000173 & $-37,77$ \\
\hline hrpL & WT hrpL & GAATGCACTTCGTCTTCCCAG & 73725 & 0,000000173 & $-38,09$ \\
\hline hrpL & WT hrpL & ACAGCCAGGTCTGCGGTTTGC & 64415 & 0,000000173 & $-40,68$ \\
\hline hrpL & WT hrpL & AAACACGCACTGGAGAATGTC & 63566 & 0,000000173 & $-34,49$ \\
\hline hrpL & WT hrpL & СТСТАССTGATGACTGACATC & 52565 & 0,000126 & $-22,9$ \\
\hline hrpL & WT hrpL & AGCAAACCGCAGACCTGGCTGT & 48156 & $6,36 \mathrm{E}-08$ & $-41,72$ \\
\hline hrpL & WT hrpL & ACACGCACTGGAGAATGTCATC & 44751 & $6,36 \mathrm{E}-08$ & $-38,4$ \\
\hline hrpL & WT hrpL & GCGCGATGCCACACAGCCAGG & 43853 & 0,000000173 & $-42,89$ \\
\hline hrpL & WT hrpL & TCAGGTTCAGCGCGATGCCAC & 42436 & 0,000000173 & $-40,62$ \\
\hline hrpL & WT hrpL & TGACTGACATCACCGTGCCCT & 42186 & 0,000000173 & $-39,77$ \\
\hline hrpL & WT hrpL & AGGGCACGGTGATGTCAGTCA & 41799 & 0,000000173 & $-41,85$ \\
\hline hrpL & WT hrpL & CTCGGAATGCACTTCGTCTTC & 39922 & 0,000000173 & $-37,38$ \\
\hline hrpL & WT hrpL & TTGGATAAACGCCCTGAGCATCTG & 39498 & 0,0000183 & $-24,63$ \\
\hline hrpL & WT hrpL & GAAGACGAAGTGCATTCCGAG & 39440 & 0,000000173 & $-36,75$ \\
\hline hrpL & WT hrpL & AACACGCACTGGAGAATGTCA & 39054 & 0,000000173 & $-35,11$ \\
\hline hrpL & WT hrpL & AACACGCACTGGAGAATGTCAT & 38433 & $6,36 \mathrm{E}-08$ & $-36,09$ \\
\hline hrpL & WT hrpL & GATGCCACACAGCCAGGTCTGC & 36555 & $6,36 \mathrm{E}-08$ & $-44,1$ \\
\hline hrpL & WT hrpL & AGGTTCAGCGCGATGCCACAC & 33405 & 0,000000173 & $-38,05$ \\
\hline hrpL & WT hrpL & GGTTCATCACCCGCTTTTGGA & 33225 & 0,000000173 & $-37,15$ \\
\hline hrpL & WT hrpL & GCGATGCCACACAGCCAGGTC & 31502 & 0,000000173 & $-43,23$ \\
\hline hrpL & WT hrpL & GCGGATCAGGTTCAGCGCGAT & 31263 & 0,000000173 & $-41,32$ \\
\hline hrpL & WT hrpL & GCAAACCGCAGACCTGGCTGT & 28863 & 0,000000173 & $-40,18$ \\
\hline hrpL & WT hrpL & CGGATCAGGTTCAGCGCGATGC & 28526 & $6,36 \mathrm{E}-08$ & $-44,14$ \\
\hline hrpL & WT hrpL & GACATTCTCCAGTGCGTGTTT & 25616 & 0,000000173 & $-36,12$ \\
\hline hrpL & WT hrpL & AAGAAACACGCACTGGAGAAT & 25143 & 0,000000173 & $-34,99$ \\
\hline hrpL & WT hrpL & AGCCAGGTCTGCGGTTTGCTG & 24514 & 0,000000173 & $-41,71$ \\
\hline hrpL & WT hrpL & GGGCACGGTGATGTCAGTCAT & 23470 & 0,000000173 & $-41,31$ \\
\hline hrpL & WT hrpL & CAGGTTCAGCGCGATGCCACA & 23400 & 0,000000173 & $-40,28$ \\
\hline hrpL & WT hrpL & ATGACTGACATCACCGTGCCC & 23337 & 0,000000173 & $-38,84$ \\
\hline hrpL & WT hrpL & CTGGCATGTTGAAACTTGTGC & 23001 & 0,000000173 & $-35,69$ \\
\hline hrpL & WT hrpL & GGCTTCAAGAAACACGCACTG & 22934 & 0,000000173 & $-37,37$ \\
\hline hrpL & WT hrpL & AAACACGCACTGGAGAATGTCATC & 19548 & $8,35 \mathrm{E}-09$ & $-39,6$ \\
\hline hrpL & WT hrpL & CCTGGCTGTGTGGCATCGCGC & 19380 & 0,000000173 & $-45,16$ \\
\hline hrpL & WT hrpL & TGACGATACATTTTGCGGAAG & 19321 & 0,000000173 & $-33,75$ \\
\hline hrpL & WT hrpL & CTTCCGCAAAATGTATCGTCA & 19136 & 0,000000173 & $-33,2$ \\
\hline hrpL & WT hrpL & GTGGCATCGCGCTGAACCTGA & 19019 & 0,000000173 & $-40,56$ \\
\hline hrpL & WT hrpL & CAGGTCTGCGGTTTGCTGGCA & 18446 & 0,000000173 & $-41,98$ \\
\hline hrpL & WT hrpL & GACGAAGTGCATTCCGAGCTGG & 18259 & $6,36 \mathrm{E}-08$ & $-41,93$ \\
\hline hrpL & WT hrpL & CCAGCTCGGAATGCACTTCGTC & 18237 & $6,36 \mathrm{E}-08$ & $-42,45$ \\
\hline hrpL & WT hrpL & GATGACATTCTCCAGTGCGTGT & 18110 & $6,36 \mathrm{E}-08$ & $-39,9$ \\
\hline hrpL & WT hrpL & GGTTCATCACCCGCTTTTGGATAA & 17376 & $8,35 \mathrm{E}-09$ & $-37,65$ \\
\hline hrpL & WT hrpL & AGCGCGATGCCACACAGCCAG & 17318 & $3 \quad 0,000000173$ & $-40,01$ \\
\hline hrpL & WT hrpL & ACGGCTGACGATACATTTTTGCGGA & 17281 & 8,35E-09 & $-43,3$ \\
\hline hrpL & WT hrpL & TCCGCAAAATGTATCGTCAGCCGT & 17224 & $8,35 \mathrm{E}-09$ & $-40,86$ \\
\hline hrpL & WT hrpL & AGGTTCAGCGCGATGCCACACA & 16921 & $6,36 \mathrm{E}-08$ & $-39,64$ \\
\hline hrpL & WT hrpL & CTGACGATACATTTTGCGGAAG & 16916 & $6,36 \mathrm{E}-08$ & $-36,32$ \\
\hline hrpL & WT hrpL & CTTCCGCAAAATGTATCGTCAG & 16855 & $6,36 \mathrm{E}-08$ & $-33,81$ \\
\hline hrpL & WT hrpL & ACGGCTGACGATACATTTTTGC & 16412 & 0,000000173 & $-35,87$ \\
\hline hrpL & WT hrpL & GCAGACCTGGCTGTGTGGCATC & 16320 & $6,36 \mathrm{E}-08$ & $-45,78$ \\
\hline hrpL & WT hrpL & GCAAAATGTATCGTCAGCCGT & 16293 & $3 \quad 0,000000173$ & $-35,49$ \\
\hline hrpL & WT hrpL & AGGGCTTCAAGAAACACGCACTGG & 16256 & $8,35 \mathrm{E}-09$ & $-45,01$ \\
\hline hrpL & WT hrpL & CAGATGCTCAGGGCGTTTATCCAA & 15961 & 0,0000183 & $-30,71$ \\
\hline hrpL & WT hrpL & TGACATTCTCCAGTGCGTGTT & 15867 & 0,000000173 & $-36,72$ \\
\hline hrpL & WT hrpL & ACGAAGTGCATTCCGAGCTGG & 15705 & 0,000000173 & $-39,04$ \\
\hline hrpL & WT hrpL & TGGCATGTTGAAACTTGTGCT & 15665 & 0,000000173 & $-34,54$ \\
\hline hrpL & WT hrpL & ATGACATTCTCCAGTGCGTGTT & 15642 & $6,36 \mathrm{E}-08$ & $-37,8$ \\
\hline hrpL & WT hrpL & CCAGCTCGGAATGCACTTCGT & 15619 & 0,000000173 & $-39,57$ \\
\hline
\end{tabular}




\begin{tabular}{|c|c|c|c|c|c|}
\hline hrpL & WT hrpL & TCCGCAAAATGTATCGTCAGC & 15009 & 0,000000173 & $-33,53$ \\
\hline hrpL & WT hrpL & CGATGCCACACAGCCAGGTCTG & 14954 & $6,36 \mathrm{E}-08$ & $-43,9$ \\
\hline hrpL & WT hrpL & CACGCACTGGAGAATGTCATC & 14901 & 0,000000173 & $-36,83$ \\
\hline hrpL & WT hrpL & GTGTGGCATCGCGCTGAACCT & 14793 & 0,000000173 & $-40,35$ \\
\hline hrpL & WT hrpL & TCAAGAAACACGCACTGGAGA & 14745 & 0,000000173 & $-37,39$ \\
\hline hrpL & WT hrpL & AGGGCTTCAAGAAACACGCACT & 14744 & 6,36E-08 & $-39,21$ \\
\hline hrpL & WT hrpL & GCTGACGATACATTTTGCGGA & 14681 & 0,000000173 & $-36,17$ \\
\hline hrpL & WT hrpL & ACGCACTGGAGAATGTCATCC & 14550 & 0,000000173 & $-36,86$ \\
\hline hrpL & WT hrpL & CACAGCCAGGTCTGCGGTTTG & 14288 & 0,000000173 & $-38,19$ \\
\hline hrpL & WT hrpL & GACCTGGCTGTGTGGCATCGC & 14183 & 0,000000173 & $-44,07$ \\
\hline hrpL & WT hrpL & TTCAAGAAACACGCACTGGAGA & 14139 & 6,36E-08 & $-38,3$ \\
\hline hrpL & WT hrpL & CTTGTGCTCGTTACGCAGGGC & 14132 & 0,000000173 & $-39,85$ \\
\hline hrpL & WT hrpL & GATCAGGTTCAGCGCGATGCC & 14062 & 0,000000173 & $-41,13$ \\
\hline hrpL & WT hrpL & ATGCCACACAGCCAGGTCTGC & 13884 & 0,000000173 & $-41,2$ \\
\hline hrpL & WT hrpL & TTACGCAGGGCTTCAAGAAAC & 13803 & 0,000000173 & $-36,3$ \\
\hline hrpL & WT hrpL & TTGCTGGCATGTTGAAACTTGTGC & 13773 & 8,35E-09 & $-41,19$ \\
\hline hrpL & WT hrpL & TCAAGAAACACGCACTGGAGAA & 13668 & $6,36 \mathrm{E}-08$ & $-38,39$ \\
\hline hrpL & WT hrpL & CCAGGTCTGCGGTTTGCTGGC & 13612 & 0,000000173 & $-42,85$ \\
\hline hrpL & WT hrpL & GCTGGCATGTTGAAACTTGTGC & 13562 & $6,36 \mathrm{E}-08$ & $-39,04$ \\
\hline hrpL & WT hrpL & ATGACTGACATCACCGTGCCCT & 13549 & $6,36 \mathrm{E}-08$ & $-40,45$ \\
\hline hrpL & WT hrpL & AGGGCACGGTGATGTCAGTCAT & 13548 & $6,36 \mathrm{E}-08$ & $-42,9$ \\
\hline hrpL & WT hrpL & TCCAAAAGCGGGTGATGAACC & 13453 & 0,000000173 & $-37,34$ \\
\hline hrpL & WT hrpL & GATCAGGTTCAGCGCGATGCCA & 13150 & $6,36 \mathrm{E}-08$ & $-42,44$ \\
\hline hrpL & WT hrpL & GCCCTTCCAGCTCGGAATGCA & 13069 & 0,000000173 & $-39,4$ \\
\hline hrpL & WT hrpL & TGCATTCCGAGCTGGAAGGGC & 12933 & 0,000000173 & $-39,23$ \\
\hline hrpL & WT hrpL & GCTGACGATACATTTTGCGGAA & 12551 & $6,36 \mathrm{E}-08$ & $-37,17$ \\
\hline hrpL & WT hrpL & TTCCGCAAAATGTATCGTCAGC & 12434 & $6,36 \mathrm{E}-08$ & $-34,53$ \\
\hline hrpL & WT hrpL & AAACACGCACTGGAGAATGTCA & 12316 & $6,36 \mathrm{E}-08$ & $-35,79$ \\
\hline hrpL & WT hrpL & TACGGCTGACGATACATTTTGC & 11375 & $6,36 \mathrm{E}-08$ & $-37,13$ \\
\hline hrpL & WT hrpL & GCAAAATGTATCGTCAGCCGTA & 11260 & $6,36 \mathrm{E}-08$ & $-36,75$ \\
\hline hrpL & WT hrpL & CGCGATGCCACACAGCCAGGTCTG & 11025 & $8,35 \mathrm{E}-09$ & $-48,97$ \\
\hline hrpL & WT hrpL & CAGCAAACCGCAGACCTGGCT & 10896 & 0,000000173 & $-40,45$ \\
\hline hrpL & WT hrpL & GAATGTCATCCACATCATCAG & 10694 & 0,000000173 & $-32,92$ \\
\hline hrpL & WT hrpL & TGTGGCATCGCGCTGAACCTG & 10665 & 0,000000173 & $-40,25$ \\
\hline hrpL & WT hrpL & CGCACTGGAGAATGTCATCCA & 10458 & 0,000000173 & $-36,01$ \\
\hline hrpL & WT hrpL & ATTCTCCAGTGCGTGTTTCTT & 10374 & 0,000000173 & $-34,96$ \\
\hline hrpL & WT hrpL & TCAGGTTCAGCGCGATGCCACA & 10185 & 6,36E-08 & $-42,18$ \\
\hline hrpL & WT hrpL & CACGCACTGGAGAATGTCATCC & 10098 & $6,36 \mathrm{E}-08$ & $-39,34$ \\
\hline hrpL & WT hrpL & ATGCACTTCGTCTTCCCAGCT & 9914 & 0,000000173 & $-38,74$ \\
\hline hrpL & WT hrpL & AGCTGGGAAGACGAAGTGCAT & 9839 & 0,000000173 & $-39,64$ \\
\hline hrpL & WT hrpL & CAAGAAACACGCACTGGAGAAT & 9800 & $6,36 \mathrm{E}-08$ & $-37,58$ \\
\hline hrpL & WT hrpL & CGCTTTTGGATAAACGCCCTGAGC & 9609 & $7,51 \mathrm{E}-08$ & $-36,44$ \\
\hline hrpL & WT hrpL & GCACAAGTTTCAACATGCCAG & 9414 & 0,000000173 & $-34,14$ \\
\hline hrpL & WT hrpL & CAGTGCGTGTTTCTTGAAGCC & 9275 & 0,000000173 & $-37,72$ \\
\hline hrpL & WT hrpL & GAAGTGCATTCCGAGCTGGAA & 9080 & 0,000000173 & $-36,71$ \\
\hline hrpL & WT hrpL & TTCCAGCTCGGAATGCACTTC & 8951 & 0,000000173 & $-36,61$ \\
\hline hrpL & WT hrpL & TCCCAGCTTTCCTGATACGGC & 8784 & 0,000000173 & $-38,98$ \\
\hline hrpL & WT hrpL & CATGTTGAAACTTGTGCTCGT & 8746 & 0,000000173 & $-33,28$ \\
\hline hrpL & WT hrpL & GATCCGCAATCACTTCCGCAA & 8649 & 0,000000173 & $-35,7$ \\
\hline hrpL & WT hrpL & GCCGTATCAGGAAAGCTGGGA & 8634 & 0,000000173 & $-40,74$ \\
\hline hrpL & WT hrpL & TTGCGGAAGTGATTGCGGATC & 8623 & 0,000000173 & $-38,19$ \\
\hline hrpL & WT hrpL & TATCGTCAGCCGTATCAGGAA & 8545 & 0,000000173 & $-36,97$ \\
\hline hrpL & WT hrpL & TTCCTGATACGGCTGACGATA & 8402 & 0,000000173 & $-36,32$ \\
\hline hrpL & WT hrpL & TGATTGCGGATCAGGTTCAGC & 8383 & 0,000000173 & $-37,55$ \\
\hline hrpL & WT hrpL & AATGTCATCCACATCATCAGG & 8354 & 0,000000173 & $-33,56$ \\
\hline hrpL & WT hrpL & TTGAAACTTGTGCTCGTTACG & 8256 & 0,000000173 & $-32,34$ \\
\hline hrpL & WT hrpL & TGCCAGCAAACCGCAGACCTG & 8247 & 0,000000173 & $-38,76$ \\
\hline hrpL & WT hrpL & GATGCCACACAGCCAGGTCT & 8129 & 0,000000468 & $-39,33$ \\
\hline hrpL & WT hrpL & CGGCTGACGATACATTTTGCGGAA & 8083 & $8,35 \mathrm{E}-09$ & $-42,69$ \\
\hline hrpL & WT hrpL & TGCTGGCATGTTGAAACTTGT & 7951 & 0,000000173 & $-35,2$ \\
\hline hrpL & WT hrpL & TTCCGCAAAATGTATCGTCAGCCG & 7926 & $8,35 \mathrm{E}-09$ & $-40,16$ \\
\hline hrpL & WT hrpL & AGACGAAGTGCATTCCGAGCT & 7925 & 0,000000173 & $-37,57$ \\
\hline hrpL & WT hrpL & GATGACATTCTCCAGTGCGTGTTT & 7839 & $8,35 \mathrm{E}-09$ & $-41,58$ \\
\hline hrpL & WT hrpL & AGCTCGGAATGCACTTCGTCT & 7798 & 0,000000173 & $-38,4$ \\
\hline hrpL & WT hrpL & CTGGCTGTGTGGCATCGCGCT & 7793 & 0,000000173 & $-43,5$ \\
\hline hrpL & WT hrpL & TGAACCTGATCCGCAATCACT & 7778 & 0,000000173 & $-36,14$ \\
\hline hrpL & WT hrpL & CAGCCAGGTCTGCGGTTTGCTG & 7735 & $6,36 \mathrm{E}-08$ & $-43,34$ \\
\hline hrpL & WT hrpL & AGTGATTGCGGATCAGGTTCA & 7705 & 0,000000173 & $-37,1$ \\
\hline hrpL & WT hrpL & TGTGTGGCATCGCGCTGAACCT & 7508 & $6,36 \mathrm{E}-08$ & $-41,93$ \\
\hline hrpL & WT hrpL & GTCATCCACATCATCAGGGTT & 7345 & 0,000000173 & $-37,28$ \\
\hline hrpL & WT hrpL & GAACCTGATCCGCAATCACTTCCG & 7275 & 8,35E-09 & $-42,84$ \\
\hline hrpL & WT hrpL & TCCACATCATCAGGGTTCATCA & 7264 & $6,36 \mathrm{E}-08$ & $-39,1$ \\
\hline hrpL & WT hrpL & CTTCCGCAAAATGTATCGTCAGCC & 7212 & $8,35 \mathrm{E}-09$ & $-40,31$ \\
\hline hrpL & WT hrpL & CGGAAGTGATTGCGGATCAGGTTC & 7182 & 8,35E-09 & $-44,6$ \\
\hline
\end{tabular}




\begin{tabular}{|c|c|c|c|c|c|}
\hline hrpL & WT hrpL & CAAAATGTATCGTCAGCCGTA & 7149 & 0,000000173 & $-33,46$ \\
\hline hrpL & WT hrpL & GGCTGACGATACATTTTGCGGAAG & 7129 & $8,35 \mathrm{E}-09$ & $-42,83$ \\
\hline hrpL & WT hrpL & TCCAGCTCGGAATGCACTTCG & 7098 & 0,000000173 & -39 \\
\hline hrpL & WT hrpL & GTGATGTCAGTCATCAGGTAG & 7088 & 0,000014 & $-26,9$ \\
\hline hrpL & WT hrpL & TTATCCAAAAGCGGGTGATGAACC & 7085 & $8,35 \mathrm{E}-09$ & $-40,39$ \\
\hline hrpL & WT hrpL & CAGCCAGGTCTGCGGTTTGC & 7081 & 0,000000468 & $-40,03$ \\
\hline hrpL & WT hrpL & TACGGCTGACGATACATTTTG & 7057 & 0,000000173 & $-33,99$ \\
\hline hrpL & WT hrpL & CGAAGTGCATTCCGAGCTGGA & 6977 & 0,000000173 & $-38,79$ \\
\hline hrpL & WT hrpL & СTACCTGATGACTGACATCAC & 6968 & 0,000014 & $-25,65$ \\
\hline hrpL & WT hrpL & CCACATCATCAGGGTTCATCA & 6913 & 0,000000173 & $-37,74$ \\
\hline hrpL & WT hrpL & ACACGCACTGGAGAATGTCA & 6894 & 0,000000468 & $-35,13$ \\
\hline hrpL & WT hrpL & CTTGTGCTCGTTACGCAGGGCT & 6867 & $6,36 \mathrm{E}-08$ & $-41,48$ \\
\hline hrpL & WT hrpL & GGATCAGGTTCAGCGCGATGC & 6816 & 0,000000173 & $-41,89$ \\
\hline hrpL & WT hrpL & GGCTGACGATACATTTTGCGG & 6725 & 0,000000173 & $-37,44$ \\
\hline hrpL & WT hrpL & CAGACCTGGCTGTGTGGCATCG & 6697 & $6,36 \mathrm{E}-08$ & $-44,82$ \\
\hline hrpL & WT hrpL & CCGCAAAATGTATCGTCAGCC & 6636 & 0,000000173 & $-35,89$ \\
\hline hrpL & WT hrpL & CAAACCGCAGACCTGGCTGTG & 6630 & 0,000000173 & $-39,45$ \\
\hline hrpL & WT hrpL & GCTCGGAATGCACTTCGTCTT & 6626 & 0,000000173 & $-38,01$ \\
\hline hrpL & WT hrpL & CAGGTTCAGCGCGATGCCAC & 6622 & 0,000000468 & $-38,73$ \\
\hline hrpL & WT hrpL & CCAGTGCGTGTTTCTTGAAGCCCT & 6609 & $8,35 \mathrm{E}-09$ & $-45,45$ \\
\hline hrpL & WT hrpL & AAGACGAAGTGCATTCCGAGC & 6534 & 0,000000173 & $-36,87$ \\
\hline hrpL & WT hrpL & GGCATCGCGCTGAACCTGATC & 6480 & 0,000000173 & -41 \\
\hline hrpL & WT hrpL & TGTATCGTCAGCCGTATCAGGAAA & 6398 & $8,35 \mathrm{E}-09$ & -42 \\
\hline hrpL & WT hrpL & CATCATCAGGGTTCATCACCC & 6376 & 0,000000173 & $-34,09$ \\
\hline hrpL & WT hrpL & ATCAGGTTCAGCGCGATGCCA & 6338 & 0,000000173 & $-39,55$ \\
\hline hrpL & WT hrpL & AGCACAAGTTTCAACATGCCA & 6336 & 0,000000173 & $-31,33$ \\
\hline hrpL & WT hrpL & TTTCCTGATACGGCTGACGATACA & 6287 & $8,35 \mathrm{E}-09$ & $-41,4$ \\
\hline hrpL & WT hrpL & CGCAAAATGTATCGTCAGCCG & 6279 & 0,000000173 & $-35,78$ \\
\hline hrpL & WT hrpL & CGGCTGACGATACATTTTTGCG & 6240 & 0,000000173 & $-36,52$ \\
\hline hrpL & WT hrpL & CAGGGCTTCAAGAAACACGCA & 6142 & 0,000000173 & $-37,82$ \\
\hline hrpL & WT hrpL & GCAGACCTGGCTGTGTGGCAT & 6060 & 0,000000173 & $-43,04$ \\
\hline hrpL & WT hrpL & GCCAGCAAACCGCAGACCTGG & 6059 & 0,000000173 & $-40,9$ \\
\hline hrpL & WT hrpL & TTGTGCTCGTTACGCAGGGCT & 6053 & 0,000000173 & $-38,99$ \\
\hline hrpL & WT hrpL & GATGACATTCTCCAGTGCGTG & 6028 & 0,000000173 & $-38,32$ \\
\hline hrpL & WT hrpL & АСCTGATCCGCAATCACTTCC & 6019 & 0,000000173 & $-37,91$ \\
\hline hrpL & WT hrpL & AGTGCGTGTTTCTTGAAGCCCT & 5992 & $6,36 \mathrm{E}-08$ & $-39,75$ \\
\hline hrpL & WT hrpL & GGAAGTGATTGCGGATCAGGT & 5960 & 0,000000173 & $-38,85$ \\
\hline hrpL & WT hrpL & TGGCATCGCGCTGAACCTGATC & 5958 & $6,36 \mathrm{E}-08$ & $-41,79$ \\
\hline hrpL & WT hrpL & ATTGCGGATCAGGTTCAGCGC & 5890 & 0,000000173 & $-38,31$ \\
\hline hrpL & WT hrpL & GGATGACATTCTCCAGTGCGT & 5860 & 0,000000173 & $-37,98$ \\
\hline hrpL & WT hrpL & TCTCCAGTGCGTGTTTCTTGA & 5801 & 0,000000173 & $-37,38$ \\
\hline hrpL & WT hrpL & GCCCTGCGTAACGAGCACAAG & 5766 & 0,000000173 & $-36,98$ \\
\hline hrpL & WT hrpL & GCACAAGTTTCAACATGCCAGCAA & 5741 & $8,35 \mathrm{E}-09$ & $-40,07$ \\
\hline hrpL & WT hrpL & TCTCCAGTGCGTGTTTCTTGAA & 5652 & $6,36 \mathrm{E}-08$ & $-38,15$ \\
\hline hrpL & WT hrpL & GCACAAGTTTCAACATGCCAGC & 5595 & $6,36 \mathrm{E}-08$ & $-37,47$ \\
\hline hrpL & WT hrpL & ACACGCACTGGAGAATGTCAT & 5577 & 0,00000052 & $-35,4$ \\
\hline hrpL & WT hrpL & TGATACGGCTGACGATACATT & 5469 & 0,000000173 & $-34,92$ \\
\hline hrpL & WT hrpL & AATGTATCGTCAGCCGTATCA & 5457 & 0,000000173 & $-34,34$ \\
\hline hrpL & WT hrpL & GTTTCTTGAAGCCCTGCGTAA & 5439 & 0,000000173 & $-36,51$ \\
\hline hrpL & WT hrpL & GCGGATCAGGTTCAGCGCGATGCC & 5402 & $8,35 \mathrm{E}-09$ & $-48,67$ \\
\hline hrpL & WT hrpL & TTCTCCAGTGCGTGTTTCTTGA & 5377 & $6,36 \mathrm{E}-08$ & $-38,37$ \\
\hline hrpL & WT hrpL & GAGAATGTCATCCACATCATC & 5304 & 0,000000173 & $-32,48$ \\
\hline hrpL & WT hrpL & GCGCGATGCCACACAGCCAGGT & 5279 & $6,36 \mathrm{E}-08$ & $-44,59$ \\
\hline hrpL & WT hrpL & TGACATTCTCCAGTGCGTGTTT & 5096 & $6,36 \mathrm{E}-08$ & $-37,62$ \\
\hline hrpL & WT hrpL & CAGACCTGGCTGTGTGGCATCGCG & 5045 & $8,35 \mathrm{E}-09$ & $-50,32$ \\
\hline hrpL & WT hrpL & CATGTTGAAACTTGTGCTCGTT & 5001 & $6,36 \mathrm{E}-08$ & $-34,1$ \\
\hline hrpL & WT hrpL & TCGTCAGCCGTATCAGGAAAG & 4978 & 0,000000173 & $-38,01$ \\
\hline hrpL & WT hrpL & CTTTCCTGATACGGCTGACGA & 4861 & 0,000000173 & $-37,31$ \\
\hline hrpL & WT hrpL & CGGATCAGGTTCAGCGCGAT & 4827 & 0,000000468 & $-38,96$ \\
\hline hrpL & WT hrpL & ACTTGTGCTCGTTACGCAGGGCTT & 4826 & 8,35E-09 & $-43,99$ \\
\hline hrpL & WT hrpL & GCCAGGTCTGCGGTTTGCTGG & 4775 & 0,000000173 & $-42,66$ \\
\hline hrpL & WT hrpL & TGCTCGTTACGCAGGGCTTCA & 4722 & 0,000000173 & $-39,46$ \\
\hline hrpL & WT hrpL & AGCGCGATGCCACACAGCCAGG & 4691 & $6,36 \mathrm{E}-08$ & $-43,31$ \\
\hline hrpL & WT hrpL & GGGCTTCAAGAAACACGCACT & 4647 & 0,000000173 & $-37,91$ \\
\hline hrpL & WT hrpL & AACCTGATCCGCAATCACTTC & 4620 & 0,000000173 & $-35,44$ \\
\hline hrpL & WT hrpL & GGATCAGGTTCAGCGCGATGCCAC & 4564 & $8,35 \mathrm{E}-09$ & $-47,79$ \\
\hline hrpL & WT hrpL & ACGCAGGGCTTCAAGAAACAC & 4564 & 0,000000173 & $-38,06$ \\
\hline hrpL & WT hrpL & TGTGGCATCGCGCTGAACCTGA & 4539 & $6,36 \mathrm{E}-08$ & $-42,15$ \\
\hline hrpL & WT hrpL & GAAGTGATTGCGGATCAGGTT & 4476 & 0,000000173 & $-36,44$ \\
\hline hrpL & WT hrpL & AAGAAACACGCACTGGAGAA & 4365 & 0,000000468 & -34 \\
\hline hrpL & WT hrpL & CCCAGCTTTCCTGATACGGCT & 4348 & 0,000000173 & $-38,77$ \\
\hline hrpL & WT hrpL & TTTCCTGATACGGCTGACGATA & 4326 & $6,36 \mathrm{E}-08$ & $-37,22$ \\
\hline hrpL & WT hrpL & AGCCGTATCAGGAAAGCTGGG & 4304 & 0,000000173 & $-39,93$ \\
\hline hrpL & WT hrpL & TGGATGACATTCTCCAGTGCG & 4286 & 0,000000173 & $-35,99$ \\
\hline
\end{tabular}




\begin{tabular}{|c|c|c|c|c|c|}
\hline hrpL & WT hrpL & TATCGTCAGCCGTATCAGGAAA & 4266 & $6,36 \mathrm{E}-08$ & $-37,85$ \\
\hline hrpL & WT hrpL & GCAGGGCTTCAAGAAACACGC & 4252 & 0,000000173 & $-39,1$ \\
\hline hrpL & WT hrpL & GCCACACAGCCAGGTCTGCGG & 4225 & 0,000000173 & $-41,67$ \\
\hline hrpL & WT hrpL & CGAGCTGGAAGGGCACGGTGA & 4176 & 0,000000173 & $-43,89$ \\
\hline hrpL & WT hrpL & CTGATGATGTGGATGACATTC & 4148 & 0,000000173 & $-34,39$ \\
\hline hrpL & WT hrpL & CAAGAAACACGCACTGGAGA & 4139 & 0,000000468 & $-35,59$ \\
\hline hrpL & WT hrpL & AACACGCACTGGAGAATGTCATC & 4071 & 2,31E-08 & $-38,86$ \\
\hline hrpL & WT hrpL & GGATGACATTCTCCAGTGCGTG & 4058 & $6,36 \mathrm{E}-08$ & $-40,57$ \\
\hline hrpL & WT hrpL & CGATGCCACACAGCCAGGTCT & 4036 & 0,000000173 & $-41,59$ \\
\hline hrpL & WT hrpL & TCACCGTGCCCTTCCAGCTCG & 3997 & 0,000000173 & $-41,32$ \\
\hline hrpL & WT hrpL & TCCACATCATCAGGGTTCATC & 3991 & 0,000000173 & $-37,51$ \\
\hline hrpL & WT hrpL & GAAACACGCACTGGAGAATGT & 3973 & 0,000000173 & $-35,64$ \\
\hline hrpL & WT hrpL & GCTCAGGGCGTTTATCCAAAAGCG & 3907 & $7,51 \mathrm{E}-08$ & $-40,54$ \\
\hline hrpL & WT hrpL & ATTCTCCAGTGCGTGTTTCTTG & 3894 & $6,36 \mathrm{E}-08$ & $-37,55$ \\
\hline hrpL & WT hrpL & CGCAAAATGTATCGTCAGCCG & 3861 & 0,00000052 & $-34,95$ \\
\hline hrpL & WT hrpL & CGGCTGACGATACATTTTGCG & 3775 & 0,00000052 & $-35,55$ \\
\hline hrpL & WT hrpL & TGACGATACATTTTGGGGAAGT & 3722 & $6,36 \mathrm{E}-08$ & $-35,43$ \\
\hline hrpL & WT hrpL & ACAAGTTTCAACATGCCAGCA & 3716 & 0,000000173 & $-34,52$ \\
\hline hrpL & WT hrpL & TGCGGATCAGGTTCAGCGCGA & 3712 & 0,000000173 & $-40,74$ \\
\hline hrpL & WT hrpL & TCCGCAAAATGTATCGTCAGCC & 3706 & 6,36E-08 & $-36,83$ \\
\hline hrpL & WT hrpL & TTTGCTGGCATGTTGAAACTT & 3675 & 0,000000173 & $-32,87$ \\
\hline hrpL & WT hrpL & AGTGATTGCGGATCAGGTTCAGCG & 3665 & 8,35E-09 & $-44,39$ \\
\hline hrpL & WT hrpL & ACGAGCACAAGTTTCAACATG & 3646 & 0,000000173 & $-33,83$ \\
\hline hrpL & WT hrpL & AGACCTGGCTGTGTGGCATC & 3607 & 0,000000468 & $-39,99$ \\
\hline hrpL & WT hrpL & GGCTGACGATACATTTTGCGGA & 3556 & 6,36E-08 & $-39,34$ \\
\hline hrpL & WT hrpL & ACTTCCGCAAAATGTATCGTCA & 3545 & $6,36 \mathrm{E}-08$ & $-34,84$ \\
\hline hrpL & WT hrpL & TTCCAGCTCGGAATGCACTTCGTC & 3515 & 8,35E-09 & $-43,56$ \\
\hline hrpL & WT hrpL & CAGCAAACCGCAGACCTGGCTG & 3513 & $6,36 \mathrm{E}-08$ & $-41,88$ \\
\hline hrpL & WT hrpL & CGGATCAGGTTCAGCGCGATGCC & 3470 & 2,31E-08 & $-45,3$ \\
\hline hrpL & WT hrpL & CCTGATGATGTGGATGACATT & 3438 & 0,000000173 & $-35,41$ \\
\hline hrpL & WT hrpL & GATCAGGTTCAGCGCGATGCCACA & 3430 & 8,35E-09 & $-46,16$ \\
\hline hrpL & WT hrpL & GACGAAGTGCATTCCGAGCTGGAA & 3403 & 8,35E-09 & $-43,62$ \\
\hline hrpL & WT hrpL & TGCATTCCGAGCTGGAAGGGCACG & 3401 & 8,35E-09 & $-45,55$ \\
\hline hrpL & WT hrpL & TTCAAGAAACACGCACTGGAGAAT & 3387 & $8,35 \mathrm{E}-09$ & $-40,26$ \\
\hline hrpL & WT hrpL & GCTTTCCTGATACGGCTGACGATA & 3387 & 8,35E-09 & $-42,87$ \\
\hline hrpL & WT hrpL & CGTAACGAGCACAAGTTTCAA & 3367 & 0,000000173 & $-32,98$ \\
\hline hrpL & WT hrpL & CGTGCCСTTCCAGCTCGGAATGCA & 3346 & 8,35E-09 & $-44,05$ \\
\hline hrpL & WT hrpL & TATCGTCAGCCGTATCAGGAAAGC & 3324 & $8,35 \mathrm{E}-09$ & $-43,44$ \\
\hline hrpL & WT hrpL & AGAAACACGCACTGGAGAATGTCA & 3250 & 8,35E-09 & $-40,28$ \\
\hline hrpL & WT hrpL & GCGGTTTGCTGGCATGTTGAAA & 3169 & $6,36 \mathrm{E}-08$ & $-39,44$ \\
\hline hrpL & WT hrpL & CATCATCAGGGTTCA & 3153 & 0,0000633 & $-24,96$ \\
\hline hrpL & WT hrpL & CTGAACCTGATCCGCAATCACT & 3141 & $6,36 \mathrm{E}-08$ & $-38,69$ \\
\hline hrpL & WT hrpL & AGTGATTGCGGATCAGGTTCAG & 3135 & $6,36 \mathrm{E}-08$ & $-39,68$ \\
\hline hrpL & WT hrpL & CAGCCAGGTCTGCGGTTTGCT & 3134 & 0,00000052 & $-41,26$ \\
\hline hrpL & WT hrpL & GCAAACCGCAGACCTGGCTG & 3076 & 0,000000468 & $-38,68$ \\
\hline hrpL & WT hrpL & TCGTCAGCCGTATCAGGAAAGC & 3056 & $6,36 \mathrm{E}-08$ & $-41,09$ \\
\hline hrpL & WT hrpL & GCTTTCCTGATACGGCTGACGA & 3048 & $6,36 \mathrm{E}-08$ & $-40,47$ \\
\hline hrpL & WT hrpL & GCAAAATGTATCGTCAGCCGTATC & 3035 & $8,35 \mathrm{E}-09$ & $-40,59$ \\
\hline hrpL & WT hrpL & TTGCTGGCATGTTGAAACTTG & 3035 & 0,000000173 & $-34,55$ \\
\hline hrpL & WT hrpL & GATACGGCTGACGATACATTTTGC & 3006 & 8,35E-09 & $-41,02$ \\
\hline hrpL & WT hrpL & TGATGAACCCTGATGATGTGGA & 3000 & $6,36 \mathrm{E}-08$ & $-39,3$ \\
\hline hrpL & WT hrpL & TGGCATCGCGCTGAACCTGAT & 2990 & 0,000000173 & $-38,94$ \\
\hline hrpL & WT hrpL & CGGCTGACGATACATTTTGCGG & 2987 & 0,000000191 & $-38,84$ \\
\hline hrpL & WT hrpL & TCTACCTGATGACTGACATC & 2978 & 0,000114 & $-22,49$ \\
\hline hrpL & WT hrpL & AACCCTGATGATGTGGATGAC & 2976 & 0,000000173 & $-36,7$ \\
\hline hrpL & WT hrpL & GTGGCATCGCGCTGAACCTG & 2962 & 0,000000468 & $-38,67$ \\
\hline hrpL & WT hrpL & CGCACTGGAGAATGTCATCCAC & 2937 & $6,36 \mathrm{E}-08$ & $-38,65$ \\
\hline hrpL & WT hrpL & CCGCAAAATGTATCGTCAGCCG & 2925 & 0,000000191 & $-37,29$ \\
\hline hrpL & WT hrpL & GGTTCAGCGCGATGCCACACA & 2921 & 0,000000173 & $-38,04$ \\
\hline hrpL & WT hrpL & TGACATTCTCCAGTGCGTGT & 2920 & 0,000000468 & $-35,95$ \\
\hline hrpL & WT hrpL & GATGTCAGTCATCAGGTAGA & 2908 & 0,000114 & $-24,92$ \\
\hline hrpL & WT hrpL & СCTTCCAGCTCGGAATGCACT & 2893 & 0,000000173 & $-38,1$ \\
\hline hrpL & WT hrpL & GCAGGGCTTCAAGAAACACGCACT & 2885 & 8,35E-09 & $-44,65$ \\
\hline hrpL & WT hrpL & GGGCTTCAAGAAACACGCACTG & 2867 & 0,000000191 & $-38,49$ \\
\hline hrpL & WT hrpL & TTTTGGATAAACGCCCTGAGC & 2856 & 0,00000156 & $-29,03$ \\
\hline hrpL & WT hrpL & CCAGGTCTGCGGTTTGCTGGCA & 2853 & $6,36 \mathrm{E}-08$ & $-44,4$ \\
\hline hrpL & WT hrpL & AGTGCATTCCGAGCTGGAAGG & 2838 & 0,000000173 & $-38,45$ \\
\hline hrpL & WT hrpL & TCTGCGGTTTGCTGGCATGTT & 2825 & 0,000000173 & $-38,07$ \\
\hline hrpL & WT hrpL & TGATGAACCCTGATGATGTGG & 2789 & 0,000000173 & $-37,67$ \\
\hline hrpL & WT hrpL & AGCCCTGCGTAACGAGCACAAG & 2788 & $6,36 \mathrm{E}-08$ & $-38,26$ \\
\hline hrpL & WT hrpL & GGGTTCATCACCCGCTTTTGG & 2783 & 0,000000173 & $-35,58$ \\
\hline hrpL & WT hrpL & CGTTACGCAGGGCTTCAAGAA & 2778 & 0,000000173 & $-37,96$ \\
\hline hrpL & WT hrpL & CCACACAGCCAGGTCTGCGGT & 2773 & 0,000000173 & $-39,84$ \\
\hline hrpL & WT hrpL & CACAGCCAGGTCTGCGGTTTGCTG & 2731 & $8,35 \mathrm{E}-09$ & $-45,72$ \\
\hline
\end{tabular}




\begin{tabular}{|c|c|c|c|c|c|}
\hline hrpL & WT hrpL & ACTTCCGCAAAATGTATCGTC & 2714 & 0,000000173 & $-33,39$ \\
\hline hrpL & WT hrpL & CGCACTGGAGAATGTCATCCA & 2696 & 0,00000052 & $-36,07$ \\
\hline hrpL & WT hrpL & CACAGCCAGGTCTGCGGTTTGC & 2684 & 6,36E-08 & $-41,57$ \\
\hline hrpL & WT hrpL & GACGATACATTTTGCGGAAGT & 2672 & 0,000000173 & $-33,86$ \\
\hline hrpL & WT hrpL & GAAACACGCACTGGAGAATGTCAT & 2662 & $8,35 \mathrm{E}-09$ & $-39,7$ \\
\hline hrpL & WT hrpL & GCGGTTTGCTGGCATGTTGAA & 2657 & 0,000000173 & $-38,54$ \\
\hline hrpL & WT hrpL & CGTGCCCTTCCAGCTCGGAAT & 2646 & 0,000000173 & $-39,41$ \\
\hline hrpL & WT hrpL & ATTCCGAGCTGGAAGGGCACG & 2637 & 0,000000173 & $-39,92$ \\
\hline hrpL & WT hrpL & TGCGTGTTTCTTGAAGCCCTG & 2587 & 0,000000173 & $-38,35$ \\
\hline hrpL & WT hrpL & AGCGCGATGCCACACAGCCAGG & 2577 & 0,000000191 & $-42,18$ \\
\hline hrpL & WT hrpL & CAGCTCGGAATGCACTTCGTCT & 2547 & 6,36E-08 & $-40,82$ \\
\hline hrpL & WT hrpL & CTTCCGCAAAATGTATCGTCAGC & 2545 & 2,31E-08 & $-37,01$ \\
\hline hrpL & WT hrpL & GCTGACGATACATTTTGCGGAAG & 2526 & 2,31E-08 & $-39,66$ \\
\hline hrpL & WT hrpL & GGGTGATGAACCCTGATGATG & 2499 & 0,000000173 & $-36,42$ \\
\hline hrpL & WT hrpL & GTATCGTCAGCCGTATCAGGA & 2494 & 0,000000173 & $-38,66$ \\
\hline hrpL & WT hrpL & TGCACTTCGTCTTCCCAGCTT & 2482 & 0,000000173 & $-38,54$ \\
\hline hrpL & WT hrpL & TGATGTCAGTCATCAGGTAGA & 2459 & 0,0000421 & $-24,65$ \\
\hline hrpL & WT hrpL & TCCTGATACGGCTGACGATAC & 2458 & 0,000000173 & $-37,98$ \\
\hline hrpL & WT hrpL & ATGTTGAAACTTGTGCTCGTT & 2452 & 0,000000173 & $-31,51$ \\
\hline hrpL & WT hrpL & AGCCCTGCGTAACGAGCACAA & 2450 & 0,000000173 & $-36,21$ \\
\hline hrpL & WT hrpL & AGACGAAGTGCATTCCGAGCTG & 2446 & 6,36E-08 & $-40,12$ \\
\hline hrpL & WT hrpL & AAGCTGGGAAGACGAAGTGCA & 2442 & 0,000000173 & $-39,45$ \\
\hline hrpL & WT hrpL & TCTACCTGATGACTGACATCA & 2425 & 0,0000421 & $-23,33$ \\
\hline hrpL & WT hrpL & ACCTGGCTGTGTGGCATCGCGC & 2420 & $6,36 \mathrm{E}-08$ & $-46,65$ \\
\hline hrpL & WT hrpL & AAGAAACACGCACTGGAGAATG & 2385 & $6,36 \mathrm{E}-08$ & $-37,24$ \\
\hline hrpL & WT hrpL & CGTCAGCCGTATCAGGAAAGC & 2378 & 0,000000173 & $-39,31$ \\
\hline hrpL & WT hrpL & AGCCAGGTCTGCGGTTTGCTGG & 2373 & $6,36 \mathrm{E}-08$ & $-44,23$ \\
\hline hrpL & WT hrpL & CGCACTGGAGAATGTCATCCA & 2371 & 0,00000052 & $-36,87$ \\
\hline hrpL & WT hrpL & GCTTTCCTGATACGGCTGACG & 2357 & 0,000000173 & $-38,58$ \\
\hline hrpL & WT hrpL & GCCAGGTCTGCGGTTTGC & 2319 & 0,00000328 & $-36,06$ \\
\hline hrpL & WT hrpL & ACATTTTGCGGAAGTGATTGCGGA & 2315 & $8,35 \mathrm{E}-09$ & $-41,41$ \\
\hline hrpL & WT hrpL & TCCGCAATCACTTCCGCAAAATGT & 2301 & $8,35 \mathrm{E}-09$ & $-38,32$ \\
\hline hrpL & WT hrpL & CACACAGCCAGGTCTGCGGTT & 2299 & 0,000000173 & $-38,18$ \\
\hline hrpL & WT hrpL & AACACGCACTGGAGAATGTCATCC & 2275 & 8,35E-09 & $-41,67$ \\
\hline hrpL & WT hrpL & ATCCACATCATCAGGGTTCAT & 2264 & 0,000000173 & $-35,71$ \\
\hline hrpL & WT hrpL & GGCTTCAAGAAACACGCACTGGAG & 2245 & 8,35E-09 & $-45,07$ \\
\hline hrpL & WT hrpL & CAGGGCTTCAAGAAACACGCAC & 2245 & $6,36 \mathrm{E}-08$ & $-40,35$ \\
\hline hrpL & WT hrpL & AGCCAGGTCTGCGGTTTGCT & 2228 & 0,000000468 & $-39,19$ \\
\hline hrpL & WT hrpL & AGGAAAGCTGGGAAGACGAAG & 2207 & 0,000000173 & $-39,03$ \\
\hline hrpL & WT hrpL & CTTCGTCTTCCCAGCTTTCCT & 2199 & 0,000000173 & $-38,58$ \\
\hline hrpL & WT hrpL & ATGACATTCTCCAGTGCGTGT & 2126 & 0,00000052 & $-37,22$ \\
\hline hrpL & WT hrpL & CCAGCAAACCGCAGACCTGGC & 2123 & 0,000000173 & $-41,21$ \\
\hline hrpL & WT hrpL & AGCGCGATGCCACACAGCCAGGTC & 2121 & 8,35E-09 & $-47,9$ \\
\hline hrpL & WT hrpL & ACCCGCTTTTGGATAAACGCCCTGA & 2109 & 0,000000025 & $-43,26$ \\
\hline hrpL & WT hrpL & GATGATGTGGATGACATTCTC & 2104 & 0,000000173 & $-34,31$ \\
\hline hrpL & WT hrpL & AGGTTCAGCGCGATGCCACACAGC & 2090 & $8,35 \mathrm{E}-09$ & $-45,32$ \\
\hline hrpL & WT hrpL & CCTGGCTGTGTGGCATCGCGCT & 2074 & $6,36 \mathrm{E}-08$ & $-46,77$ \\
\hline hrpL & WT hrpL & AACGAGCACAAGTTTCAACATG & 2060 & $6,36 \mathrm{E}-08$ & $-34,66$ \\
\hline hrpL & WT hrpL & CAGGTCTGCGGTTTGCTGGC & 2045 & 0,000000468 & $-40,59$ \\
\hline hrpL & WT hrpL & TCACCCGCTTTTGGATAAACG & 2044 & 0,000000173 & $-31,31$ \\
\hline hrpL & WT hrpL & AGCTTTCCTGATACGGCTGAC & 2027 & 0,000000173 & $-37,32$ \\
\hline hrpL & WT hrpL & ACACAGCCAGGTCTGCGGTTTG & 2023 & 6,36E-08 & $-39,22$ \\
\hline hrpL & WT hrpL & CACGCACTGGAGAATGTCAT & 2009 & 0,000000468 & $-34,33$ \\
\hline hrpL & WT hrpL & ACTTCCGCAAAATGTATCGTCAGC & 2007 & 8,35E-09 & $-38,32$ \\
\hline hrpL & WT hrpL & CCGCAGACCTGGCTGTGTGGC & 2007 & 0,000000173 & $-45,37$ \\
\hline hrpL & WT hrpL & CATCATCAGGGTTCATCA & 1987 & 0,00000328 & $-30,49$ \\
\hline hrpL & WT hrpL & GCTCGGAATGCACTTCGTCTTC & 1983 & 6,36E-08 & $-40,74$ \\
\hline hrpL & WT hrpL & CGGAAGTGATTGCGGATCAGG & 1979 & 0,000000173 & $-39,54$ \\
\hline hrpL & WT hrpL & CCTGATCCGCAATCACTTCCG & 1973 & 0,000000173 & $-38,19$ \\
\hline hrpL & WT hrpL & GTCAGCCGTATCAGGAAAGCT & 1970 & 0,000000173 & $-37,81$ \\
\hline hrpL & WT hrpL & СССTTCCAGCTCGGAATGCACT & 1968 & $6,36 \mathrm{E}-08$ & $-41,07$ \\
\hline hrpL & WT hrpL & CACTTCCGCAAAATGTATCGT & 1960 & 0,000000173 & $-32,47$ \\
\hline hrpL & WT hrpL & GCTGACGATACATTTTGCGGAAGT & 1956 & 8,35E-09 & $-41,32$ \\
\hline hrpL & WT hrpL & GCTTCAAGAAACACGCACTGGA & 1956 & 6,36E-08 & $-39,57$ \\
\hline hrpL & WT hrpL & AAGCCCTGCGTAACGAGCACAAGT & 1943 & 8,35E-09 & $-40,69$ \\
\hline hrpL & WT hrpL & ACACGCACTGGAGAATGTCATC & 1943 & 0,000000191 & $-36,28$ \\
\hline hrpL & WT hrpL & TCACCGTGCCCTTCCAGCTCG & 1926 & 0,00000052 & $-40,91$ \\
\hline hrpL & WT hrpL & AGTGCATTCCGAGCTGGAAGGG & 1922 & $6,36 \mathrm{E}-08$ & $-41,71$ \\
\hline hrpL & WT hrpL & CGCACTGGAGAATGTC & 1922 & 0,0000253 & $-28,28$ \\
\hline hrpL & WT hrpL & GGCTTCAAGAAACACGCACTGG & 1920 & $6,36 \mathrm{E}-08$ & $-40,64$ \\
\hline hrpL & WT hrpL & CATCATCAGGGTTCATC & 1918 & 0,00000985 & $-28,92$ \\
\hline hrpL & WT hrpL & AACTTGTGCTCGTTACGCAGGGCT & 1911 & 8,35E-09 & $-43,85$ \\
\hline hrpL & WT hrpL & ATCACTTCCGCAAAATGTATC & 1910 & 0,000000173 & $-30,82$ \\
\hline hrpL & WT hrpL & AGTGCGTGTTTCTTGAAGCCC & 1904 & 0,000000173 & $-38,25$ \\
\hline
\end{tabular}




\begin{tabular}{|c|c|c|c|c|c|}
\hline hrpL & WT hrpL & CTGGCATGTTGAAACTTGTGCT & 1884 & $6,36 \mathrm{E}-08$ & $-37,14$ \\
\hline hrpL & WT hrpL & ACGATACATTTTGCGGAAGTG & 1884 & 0,000000173 & $-33,56$ \\
\hline hrpL & WT hrpL & TGCCACACAGCCAGGTCTGCG & 1862 & 0,000000173 & $-41,05$ \\
\hline hrpL & WT hrpL & GAAGACGAAGTGCATTCCGAGC & 1861 & $6,36 \mathrm{E}-08$ & $-39,74$ \\
\hline hrpL & WT hrpL & TGAAGCCCTGCGTAACGAGCA & 1854 & 0,000000173 & $-36,35$ \\
\hline hrpL & WT hrpL & TGCGGATCAGGTTCAGCGCGAT & 1844 & 6,36E-08 & $-41,84$ \\
\hline hrpL & WT hrpL & AAGAAACACGCACTGGAGAATGTC & 1836 & 8,35E-09 & $-39,88$ \\
\hline hrpL & WT hrpL & CATCATCAGGGTTCAT & 1836 & 0,0000253 & $-26,05$ \\
\hline hrpL & WT hrpL & AGAAACACGCACTGGAGAATGT & 1833 & $6,36 \mathrm{E}-08$ & $-37,24$ \\
\hline hrpL & WT hrpL & GCCGTATCAGGAAAGCTGGGAA & 1823 & $6,36 \mathrm{E}-08$ & $-41,74$ \\
\hline hrpL & WT hrpL & AGACCTGGCTGTGTGGCATCG & 1823 & 0,000000173 & $-42,32$ \\
\hline hrpL & WT hrpL & AGGGCTTCAAGAAACACGCAC & 1816 & 0,000000173 & $-37,76$ \\
\hline hrpL & WT hrpL & CCACACAGCCAGGTCTGCGGTTTG & 1813 & $8,35 \mathrm{E}-09$ & $-44,22$ \\
\hline hrpL & WT hrpL & GTGTTTCTTGAAGCCCTGCGT & 1803 & 0,000000173 & $-38,5$ \\
\hline hrpL & WT hrpL & ATGCCACACAGCCAGGTCTGCGGT & 1793 & 8,35E-09 & $-45,58$ \\
\hline hrpL & WT hrpL & TGTGCTCGTTACGCAGGGCTT & 1790 & 0,000000173 & $-38,91$ \\
\hline hrpL & WT hrpL & GCGTGTTTCTTGAAGCCCTGC & 1783 & 0,000000173 & $-40,13$ \\
\hline hrpL & WT hrpL & CGAGCTGGAAGGGCACGGTGA & 1780 & 0,00000052 & $-43,49$ \\
\hline hrpL & WT hrpL & TTCCCAGCTTTCCTGATACGGC & 1779 & $6,36 \mathrm{E}-08$ & $-39,96$ \\
\hline hrpL & WT hrpL & GATACATTTTGCGGAAGTGAT & 1774 & 0,000000173 & $-32,49$ \\
\hline hrpL & WT hrpL & TTCTCCAGTGCGTGTTTCTT & 1734 & 0,000000468 & $-33,96$ \\
\hline hrpL & WT hrpL & GGATAAACGCCCTGAGCATCTG & 1715 & 0,000139 & $-22,42$ \\
\hline hrpL & WT hrpL & CAGCCAGGTCTGCGGTTTGCT & 1714 & 0,00000156 & $-35,44$ \\
\hline hrpL & WT hrpL & TGCGGTTTGCTGGCATGTTGA & 1691 & 0,000000173 & $-38,31$ \\
\hline hrpL & WT hrpL & TTGCGGATCAGGTTCAGCGCG & 1689 & 0,000000173 & $-39,84$ \\
\hline hrpL & WT hrpL & TTTGGATAAACGCCCTGAGCATCT & 1686 & 0,00000608 & $-26,22$ \\
\hline hrpL & WT hrpL & ACATTCTCCAGTGCGTGTTTC & 1667 & 0,000000173 & $-36,31$ \\
\hline hrpL & WT hrpL & TGTTGAAACTTGTGCTCGTTA & 1662 & 0,000000173 & $-31,66$ \\
\hline hrpL & WT hrpL & AAAGCTGGGAAGACGAAGTGCATT & 1661 & 8,35E-09 & $-42,31$ \\
\hline hrpL & WT hrpL & CTTTTGGATAAACGCCCTGAGC & 1658 & 0,000000572 & $-31,53$ \\
\hline hrpL & WT hrpL & AATGCACTTCGTCTTCCCAGCTTT & 1657 & $8,35 \mathrm{E}-09$ & $-41,42$ \\
\hline hrpL & WT hrpL & TCTCCAGTGCGTGTTTCTTG & 1653 & 0,000000468 & $-35,55$ \\
\hline hrpL & WT hrpL & CGCAGGGCTTCAAGAAACACGC & 1647 & $6,36 \mathrm{E}-08$ & $-40,12$ \\
\hline hrpL & WT hrpL & GATGACATTCTCCAGTGCGTGTT & 1640 & 2,31E-08 & $-40,68$ \\
\hline hrpL & WT hrpL & TCAGCGCGATGCCACACAGCCAGG & 1628 & $8,35 \mathrm{E}-09$ & $-47,33$ \\
\hline hrpL & WT hrpL & ATCAGGTTCAGCGCGATGCCAC & 1628 & $6,36 \mathrm{E}-08$ & $-41,72$ \\
\hline hrpL & WT hrpL & CTGAACCTGATCCGCAATCAC & 1626 & 0,000000173 & $-37,09$ \\
\hline hrpL & WT hrpL & ACCCGCTTTTGGATAAACGCCCTG & 1617 & 8,35E-09 & $-42,39$ \\
\hline hrpL & WT hrpL & CAGCGCGATGCCACACAGCCA & 1613 & 0,000000173 & $-39,8$ \\
\hline hrpL & WT hrpL & CGCGATGCCACACAGCCAGGT & 1613 & 0,000000173 & $-42,31$ \\
\hline hrpL & WT hrpL & AAATGTATCGTCAGCCGTATCAGG & 1602 & 8,35E-09 & -41 \\
\hline hrpL & WT hrpL & GGTTTGCTGGCATGTTGAAAC & 1599 & 0,000000173 & $-36,18$ \\
\hline hrpL & WT hrpL & GATGAACCCTGATGATGTGGA & 1595 & 0,000000173 & $-37,72$ \\
\hline hrpL & WT hrpL & TGTGGCATCGCGCTGAACCTGATC & 1593 & $8,35 \mathrm{E}-09$ & $-46,07$ \\
\hline hrpL & WT hrpL & GAAACTTGTGCTCGTTACGCA & 1591 & 0,000000173 & $-33,84$ \\
\hline hrpL & WT hrpL & GTGATTGCGGATCAGGTTCAG & 1589 & 0,000000173 & $-38,09$ \\
\hline hrpL & WT hrpL & CCTGATACGGCTGACGATACATTT & 1569 & $8,35 \mathrm{E}-09$ & $-41,23$ \\
\hline hrpL & WT hrpL & AGCCGTATCAGGAAAGCTGGGA & 1555 & $6,36 \mathrm{E}-08$ & $-41,82$ \\
\hline hrpL & WT hrpL & GTTTGCTGGCATGTTGAAACT & 1552 & 0,000000173 & $-34,54$ \\
\hline hrpL & WT hrpL & GATGCCACACAGCCAGGTCTGCGG & 1543 & 8,35E-09 & $-47,26$ \\
\hline hrpL & WT hrpL & TTCAAGAAACACGCACTGGAG & 1542 & 0,000000173 & $-36,42$ \\
\hline hrpL & WT hrpL & TCCCAGCTTTCCTGATACGGCT & 1540 & $6,36 \mathrm{E}-08$ & $-40,57$ \\
\hline hrpL & WT hrpL & TACGGCTGACGATACATTTTGC & 1535 & 0,000000191 & $-34,98$ \\
\hline hrpL & WT hrpL & GTGCCCTTCCAGCTCGGAATG & 1531 & 0,000000173 & $-39,48$ \\
\hline hrpL & WT hrpL & AAGTTTCAACATGCCAGCAAA & 1527 & 0,000000173 & $-32,73$ \\
\hline hrpL & WT hrpL & GCGCGATGCCACACAGCCAGGTCT & 1525 & 8,35E-09 & $-49,04$ \\
\hline hrpL & WT hrpL & CATTCCGAGCTGGAAGGGCAC & 1511 & 0,000000173 & $-40,19$ \\
\hline hrpL & WT hrpL & TACGCAGGGCTTCAAGAAACACGC & 1499 & $8,35 \mathrm{E}-09$ & $-42,63$ \\
\hline hrpL & WT hrpL & ACGCAGGGCTTCAAGAAACACG & 1485 & $6,36 \mathrm{E}-08$ & $-39,99$ \\
\hline hrpL & WT hrpL & ATTCTCCAGTGCGTGTTTCTTGAA & 1483 & $8,35 \mathrm{E}-09$ & $-40,13$ \\
\hline hrpL & WT hrpL & ATGCCACACAGCCAGGTCTG & 1475 & 0,000000468 & $-38,7$ \\
\hline hrpL & WT hrpL & TGTGTGGCATCGCGCTGAACC & 1473 & 0,000000173 & $-40,15$ \\
\hline hrpL & WT hrpL & GCCAGGTCTGCGGTTTGCT & 1472 & 0,00000125 & $-37,73$ \\
\hline hrpL & WT hrpL & TGCTGGCATGTTGAAACTTGTGC & 1454 & 2,31E-08 & $-40,19$ \\
\hline hrpL & WT hrpL & CTTTTGGATAAACGCCCTGAGCAT & 1416 & 0,000000676 & $-30,17$ \\
\hline hrpL & WT hrpL & CAAGAAACACGCACTGGAGAATGT & 1396 & 8,35E-09 & $-40,74$ \\
\hline hrpL & WT hrpL & GCAAAATGTATCGTCAGCCGTA & 1394 & 0,000000191 & $-35,02$ \\
\hline hrpL & WT hrpL & AGAAACACGCACTGGAGAATG & 1375 & 0,000000173 & $-36,34$ \\
\hline hrpL & WT hrpL & TTTCAACATGCCAGCAAACCGC & 1372 & $6,36 \mathrm{E}-08$ & $-37,69$ \\
\hline hrpL & WT hrpL & TTCAAGAAACACGCACTGGAG & 1371 & 0,00000052 & $-36,05$ \\
\hline hrpL & WT hrpL & CACATCATCAGGGTTCATCAC & 1345 & 0,000000173 & $-37,17$ \\
\hline hrpL & WT hrpL & GCCACACAGCCAGGTCTGCGGT & 1343 & $6,36 \mathrm{E}-08$ & $-42,88$ \\
\hline hrpL & WT hrpL & CTTGTGCTCGTTACGCAGGGCTT & 1334 & 2,31E-08 & $-42,38$ \\
\hline hrpL & WT hrpL & TCAGGTTCAGCGCGATGCCAC & 1329 & 0,00000052 & $-40,22$ \\
\hline
\end{tabular}




\begin{tabular}{|c|c|c|c|c|c|}
\hline hrpL & WT hrpL & TGACATTCTCCAGTGCGTGTTTCT & 1325 & 8,35E-09 & $-42,07$ \\
\hline hrpL & WT hrpL & AGCAAACCGCAGACCTGGCTG & 1313 & 0,00000052 & $-39,66$ \\
\hline hrpL & WT hrpL & GGGCTTCAAGAAACACGCACTG & 1305 & $6,36 \mathrm{E}-08$ & $-40,49$ \\
\hline hrpL & WT hrpL & CAAGTTTCAACATGCCAGCAA & 1305 & 0,000000173 & $-34,51$ \\
\hline hrpL & WT hrpL & ATTGCGGATCAGGTTCAGCGCG & 1302 & 0,000000191 & $-40,06$ \\
\hline hrpL & WT hrpL & CACGCACTGGAGAATGTCATCCAC & 1299 & 8,35E-09 & $-42,91$ \\
\hline hrpL & WT hrpL & ACGCACTGGAGAATGTCATC & 1293 & 0,000000468 & $-34,83$ \\
\hline hrpL & WT hrpL & TGATCCGCAATCACTTCCGCA & 1292 & 0,000000173 & $-36,63$ \\
\hline hrpL & WT hrpL & TGAACCCTGATGATG & 1289 & 0,0000633 & $-24,88$ \\
\hline hrpL & mut hrpL & TTGGGAGGATGAGGTTCACTC & 74216 & 0,031 & $-23,24$ \\
\hline hrpL & mut hrpL & AGTTGGGAGGATGAGGTTCAC & 9839 & 0,031 & $-27,67$ \\
\hline hrpL & mut hrpL & TCCСAACTCTCTTGGTAAGGT & 8784 & 0,031 & $-18,25$ \\
\hline hrpL & mut hrpL & ACCTTACCAAGAGAGTTGGGA & 8634 & 0,031 & $-19,2$ \\
\hline hrpL & mut hrpL & TGACGGCCGATCAGATTCAGA & 8383 & 0,003 & $-17,72$ \\
\hline hrpL & mut hrpL & TGAATCTGATCGGCCGTCATT & 7778 & 0,01 & $-23,16$ \\
\hline hrpL & mut hrpL & AATGACGGCCGATCAGATTCA & 7705 & 0,01 & $-15,67$ \\
\hline hrpL & mut hrpL & TCAACGTCGTCCGGATTCATAA & 7264 & 0,034 & $-17,09$ \\
\hline hrpL & mut hrpL & CAACGTCGTCCGGATTCATAA & 6913 & 0,092 & $-17,42$ \\
\hline hrpL & mut hrpL & CGTCGTCCGGATTCATAACTC & 6376 & 0,092 & $-12,82$ \\
\hline hrpL & mut hrpL & AATCTGATCGGCCGTCATTTG & 4620 & 0,031 & $-20,67$ \\
\hline hrpL & mut hrpL & СССАACTCTCTTGGTAAGGTT & 4348 & 0,092 & $-17,15$ \\
\hline hrpL & mut hrpL & AACCTTACCAAGAGAGTTGGG & 4304 & 0,092 & $-16,79$ \\
\hline hrpL & mut hrpL & TCAACGTCGTCCGGATTCATA & 3991 & 0,031 & $-17,1$ \\
\hline hrpL & mut hrpL & CGTCGTCCGGATTCA & 3153 & 0,42 & $-12,84$ \\
\hline hrpL & mut hrpL & CTGAATCTGATCGGCCGTCATT & 3141 & 0,004 & $-25,72$ \\
\hline hrpL & mut hrpL & AATGACGGCCGATCAGATTCAG & 3135 & 0,004 & $-18,26$ \\
\hline hrpL & mut hrpL & GAGTTGGGAGGATGAGGTTCA & 2442 & 0,031 & $-28,38$ \\
\hline hrpL & mut hrpL & GTCAACGTCGTCCGGATTCAT & 2264 & 0,031 & $-17,4$ \\
\hline hrpL & mut hrpL & AAGAGAGTTGGGAGGATGAGG & 2207 & 0,092 & $-30,43$ \\
\hline hrpL & mut hrpL & CGTCGTCCGGATTCATAA & 1987 & 0,19 & $-14,97$ \\
\hline hrpL & mut hrpL & CGTCGTCCGGATTCATA & 1918 & 0,19 & -15 \\
\hline hrpL & mut hrpL & CGTCGTCCGGATTCAT & 1836 & 0,17 & $-13,93$ \\
\hline hrpL & mut hrpL & AGAGTTGGGAGGATGAGGTTCACT & 1661 & 0,04 & $-29,64$ \\
\hline hrpL & mut hrpL & CTGAATCTGATCGGCCGTCAT & 1626 & 0,003 & $-25,01$ \\
\hline hrpL & mut hrpL & ATGACGGCCGATCAGATTCAG & 1589 & 0,003 & $-18,26$ \\
\hline hrpL & mut hrpL & AACCTTACCAAGAGAGTTGGGA & 1555 & 0,034 & $-18,68$ \\
\hline hrpL & mut hrpL & TCCCAACTCTCTTGGTAAGGTT & 1540 & 0,034 & $-18,94$ \\
\hline hrpL & mut hrpL & AACGTCGTCCGGATTCATAAC & 1345 & 0,031 & $-17,46$ \\
\hline hrpL & mut hrpL & TGAATCCGGACGACG & 1289 & 0,42 & $-10,13$ \\
\hline
\end{tabular}




\begin{tabular}{|c|c|c|}
\hline Oligo name & Oligo Sequence (5'-3') & Usage \\
\hline GyrA-F & AACTGCTGGGTGAGTACCA & \multirow{2}{*}{ qPCR of bacterial transcript $g y r A$} \\
\hline GyrA-R & GAGCTCTTCGCGGATCACT & \\
\hline ProC-F & CGCAGATGATGAAAAGCGTC & \multirow{2}{*}{ qPCR of bacterial transcript proC } \\
\hline Proc-R & AGTCAGGCTGGCACAGGTG & \\
\hline RpoB-F & GTAGGTCTGGTCCGTGTTGA & \multirow{2}{*}{ qPCR of bacterial transcript $r p o B$} \\
\hline RpoB-R & GCAAGTAATCTCGGACAGCG & \\
\hline Cfa6-F & GTCTTCATCTTTCCCGGTCA & \multirow{2}{*}{ qPCR of bacterial transcript $c f a 6$} \\
\hline Cfa6-R & GTCTCGATCTGGTCGATGGT & \\
\hline HrpL-F & CGAGTCATTCAGGCCATTGATT & \multirow{2}{*}{ qPCR of bacterial transcript $h r p L$} \\
\hline HrpL-R & GTTTCCTGATAATTGCCGTCCA & \\
\hline $\operatorname{luxA}-\mathrm{F}$ & CGGAGTTTGGTTTGCTTGGT & \multirow{2}{*}{ qPCR of bacterial transcript luxA } \\
\hline luxA-R & CAAGTTGGCGTACTGGATGG & \\
\hline luxB-F & GCGGAGGAAGCTTGCTTATT & \multirow{2}{*}{ qPCR of bacterial transcript luxB } \\
\hline luxB-R & TGATATTCAACCGGGCGATT & \\
\hline Nb-IUXA/LUXB-F & TGCTTACATACCAACCTCCCCAATT & Template DNA amplification for siRNA probe preparation \\
\hline Nb-IUXA/LUXB-R & GGCGCGCCCAGGATGATGAGTTGTA & for northern blot analysis \\
\hline $\mathrm{Nb}-\mathrm{CFA6- \textrm {F }}$ & CAACAACTTGCCGGGATCCTG & Template DNA amplification for siRNA probe preparation \\
\hline $\mathrm{Nb}-\mathrm{CFA6-R}$ & GGCTTGCATCCGACAGAATCAG & for northern blot analysis \\
\hline $\mathrm{Nb}-\mathrm{HRPL}-\mathrm{F}$ & CACTTCGTCTTCCCAGCTTTC & Template DNA amplification for siRNA probe preparation \\
\hline $\mathrm{Nb}-\mathrm{HRPL}-\mathrm{R}$ & TTTATCCAAAAGCGGGTGATGAAC & for northern blot analysis \\
\hline $\mathrm{Nb}-\mathrm{CYP} 51-\mathrm{F}$ & ATCCGAGCATGACATGATGA & Template DNA amplification for siRNA probe preparation \\
\hline $\mathrm{Nb}-\mathrm{CYP} 51-\mathrm{R}$ & GTACGGGTCGATGCCATATT & for northern blot analysis \\
\hline U6 & AGGGGCCATGCTAATCTTCTC & probe for northern blot analysis \\
\hline tomato-Ubi-F & GGACGGACGTACTCTAGCTGAT & qPCR on genomic DNA from Tomato infected with Pto \\
\hline tomato-Ubi-F & AGCTTTCGACCTCAAGGGTA & DC3000 \\
\hline Pto-GFP-F & TGGAAGCGTTCAACTAGCAG & qPCR on genomic DNA from Tomato infected with Pto \\
\hline Pto-GFP-R & AAAGGGCAGATTGTGTGGAC & DC3000 \\
\hline IR-CFA6/HRPL-F & GTTCATCACCCGCTTTTGGA & \multirow{2}{*}{ qPCR of the IR-CFA6/HRPL precursor } \\
\hline IR-CFA6/HRPL-R & ССССТСТTТСТАССТTСССА & \\
\hline Ath-Ubi-F & TGAAGTCGTGAGACAGCGTTG & \multirow{2}{*}{ qPCR of the IR-CFA6/HRPL precursor } \\
\hline Ath-Ubi-F & GGGCTTTCTCATTGTTGGTC & \\
\hline HRPL-pDON207-F & AAAAAGCAGGCTTCATGTTTCAGAAGATTGTGATC & \multirow{2}{*}{ Cloning of WT HRPL and mut HRPL in pDON207-attB1/B2 } \\
\hline HRPL-pDON207-R & AGAAAGCTGGGTCGGCGAACGGGTCGATTTGCTG & \\
\hline IR-HRPL-F & aGGTCTCaCTGCCTGATGACTGACATCACCGTG & \multirow{3}{*}{ Cloning of IR-HRPL region in a "E" Green Gate module } \\
\hline IR-HRPL-R & aGGTCTCaTAGTCAGGGCGTTTATCCAAAAGCG & \\
\hline dcl2-1-WT-FP & TGAATCATCTGGAAGAGGTGG & \\
\hline dcl2-1-mut-FP & ATTTTGCCGATTTCGGAAC & \multirow[t]{3}{*}{ genotyping of $d c / 2-1$ allele } \\
\hline dcI2-1-WT-RP & TGAATCATCTGGAAGAGGTGG & \\
\hline dcl3-1-LP & ACAGGTAACCTTGCCATGTTG & \\
\hline dcl3-1-RP & TGGAAAAGTTTGCTACAACGG & \multirow[t]{3}{*}{ genotyping of $d c / 3-1$ allele } \\
\hline LBa1 & TGGTTCACGTAGTGGGCCATCG & \\
\hline dcl4-2-G8605-FP & GGCTGCACAGCTGATGATTACAA & \\
\hline dcl4-2-G9512-RP & GCCGCTCGAGATCATCAGCAAAGGAAT & \multirow[t]{2}{*}{ genotyping of $d c / 4-2$ allele } \\
\hline GABI-8474-LP & ATAATAACGCTGCGGACATCTACATTTT & \\
\hline
\end{tabular}

